\title{
Practical Asymmetric Synthesis of Chiral Sulfoximines via Sulfur-selective Alkylation
}

\author{
Yoshiaki Maeda, ${ }^{\#[a]}$ Suguru Hamada, ${ }^{\#[a]}$ Yusuke Aota, ${ }^{[a]}$ Kazuya Otsubo, ${ }^{[a]}$ \\ Taichi Kano, ${ }^{*[a, b]}$ and Keiji Maruoka*[c,d]
}

[a] Department of Chemistry, Graduate School of Science, Kyoto University, Sakyo, Kyoto 606-8502 (Japan)

[b] Department of Applied Chemistry, Graduate School of Engineering, Tokyo University of Agriculture and Technology, Koganei, Tokyo, 184-8588 (Japan) [c] Graduate School of Pharmaceutical Sciences, Kyoto University, Sakyo, Kyoto 6068501 (Japan)

[d] School of Chemical Engineering and Light Industry, Guangdong University of Technology, Guangzhou 510006 (China)

\footnotetext{
*E-mail : kano@go.tuat.ac.jp

*E-mail : maruoka.keiji.4w@kyoto-u.ac.jp

\# These authors contributed equally.
} 


\section{Table of Contents}

1. ${ }^{1} \mathrm{H}$ NMR and ${ }^{13} \mathrm{C}\left\{{ }^{1} \mathrm{H}\right\}$ NMR Spectra

2. ${ }^{1} \mathrm{H}$ NMR spectra of NMR experiment $\quad$ S33

3. HPLC charts of mechanistic investigation for racemization S42

$\begin{array}{ll}\text { 4. HPLC charts of compounds } & \text { S51 }\end{array}$

$\begin{array}{ll}\text { 5. ORTEP drawing of compound } 4 & \text { S80 }\end{array}$ 
${ }^{1} \mathrm{H}$ NMR and ${ }^{13} \mathrm{C}\left\{{ }^{1} \mathrm{H}\right\}$ NMR Spectra

${ }^{1}$ H NMR of Scheme 2 (mechanistic investigation for racemization)
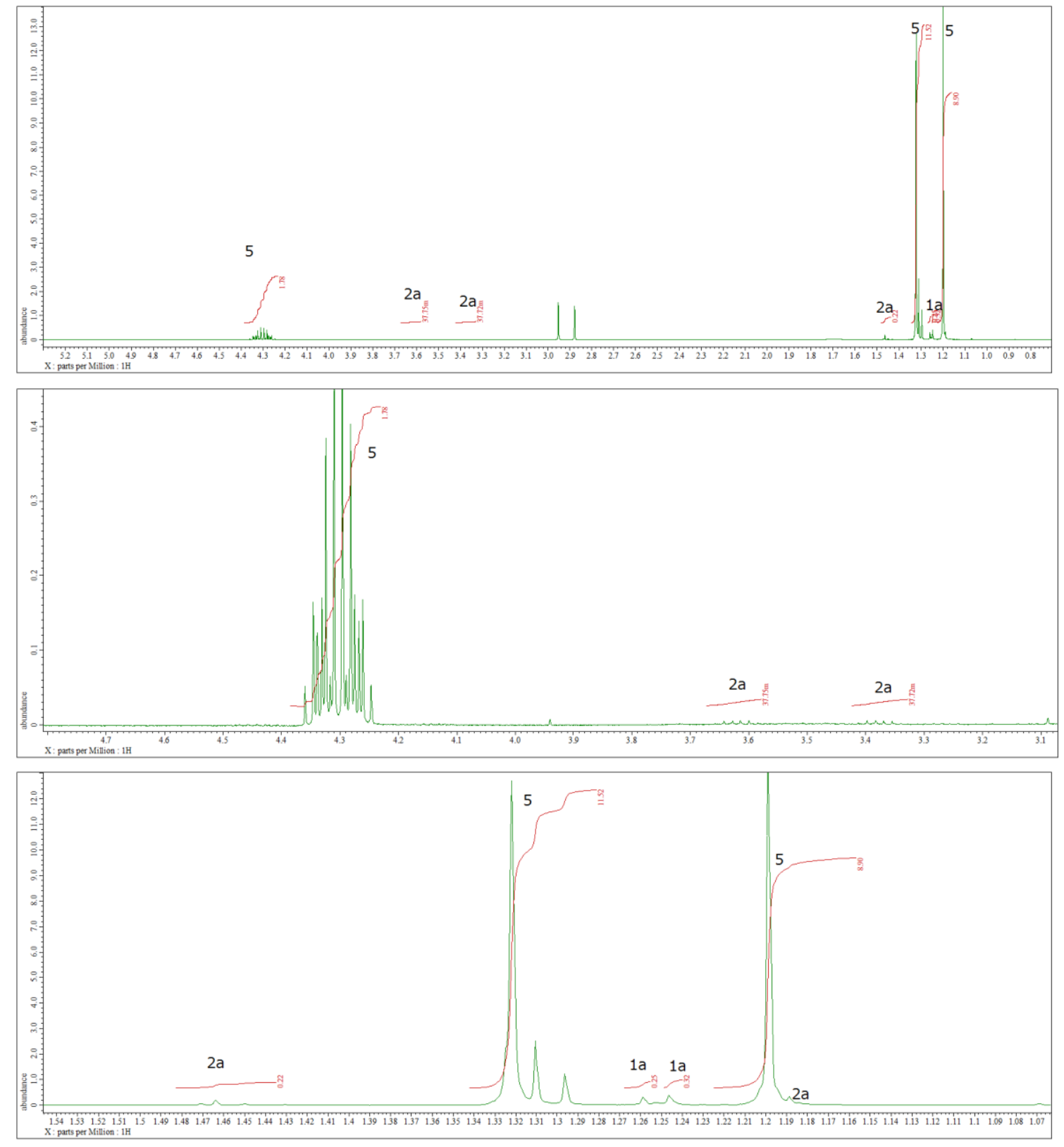
Sodium salt of 1a (3)

${ }^{1} \mathrm{H}$ NMR (500 MHz, DMSO-d6)
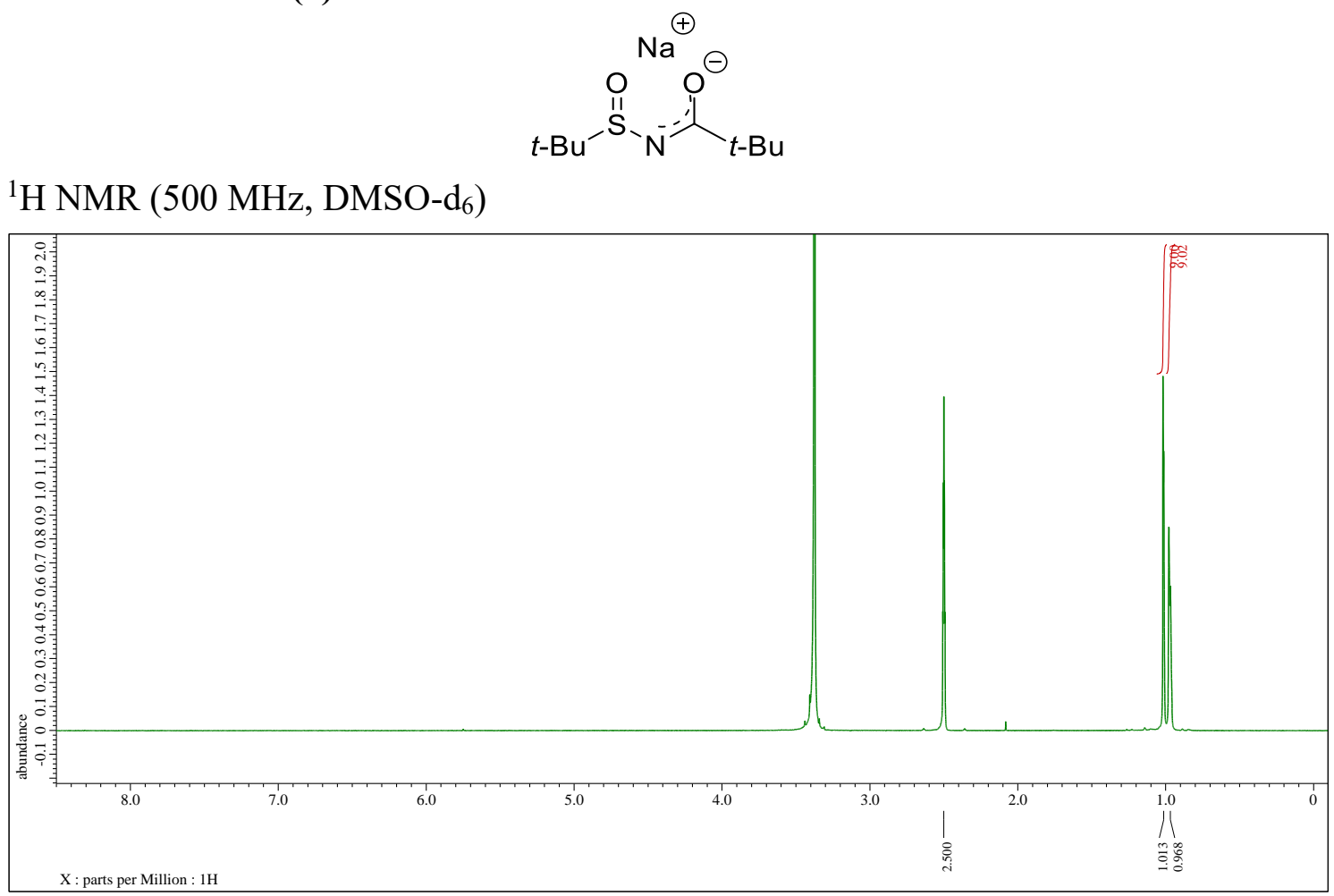

${ }^{13} \mathrm{C}\left\{{ }^{1} \mathrm{H}\right\}$ NMR (125 MHz, DMSO-d 6 )

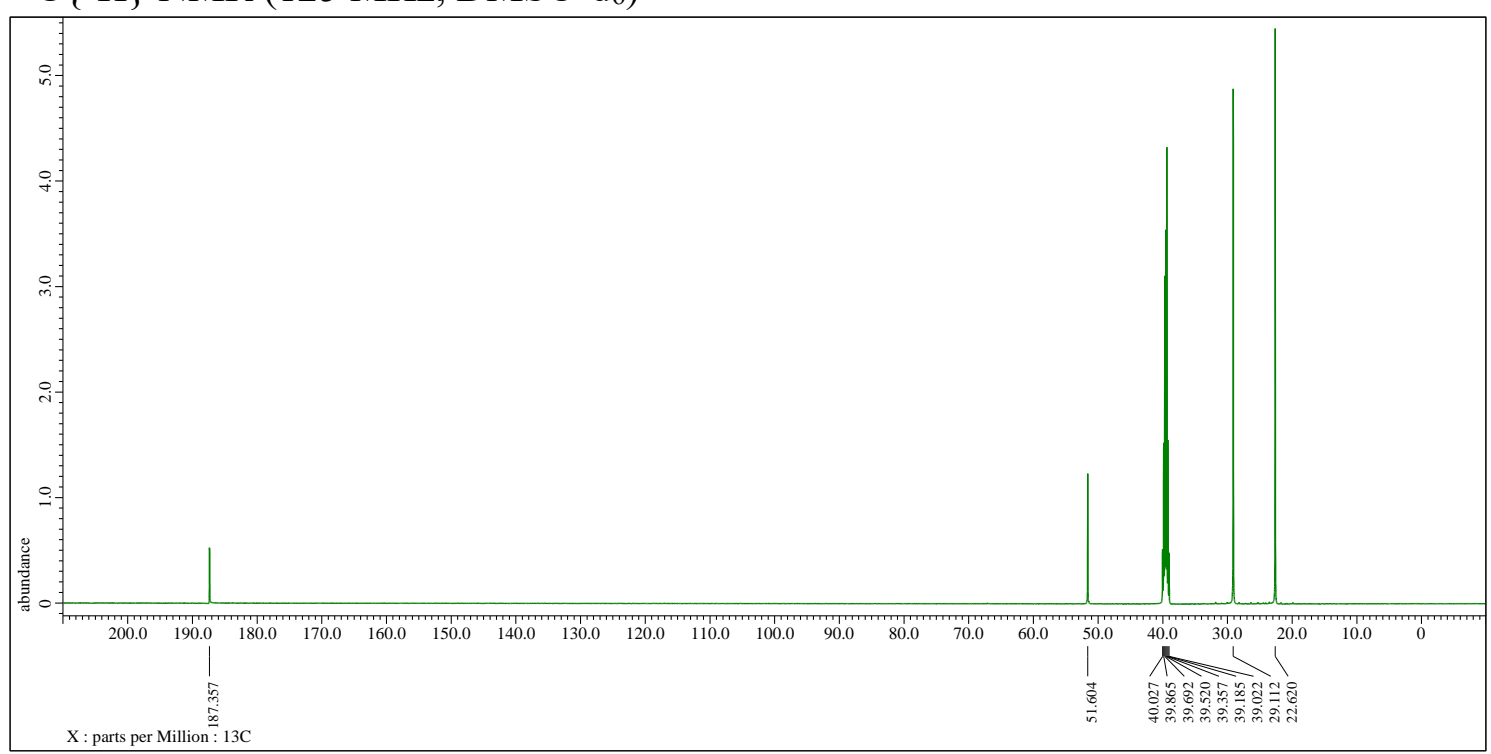




\section{Complex 4}

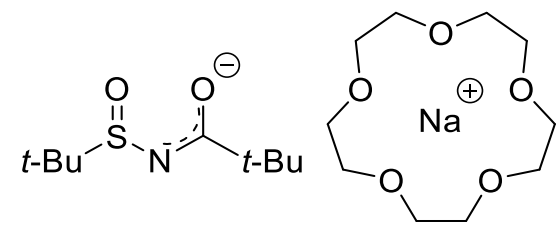

${ }^{1} \mathrm{H}$ NMR (500 MHz, THF-d8)
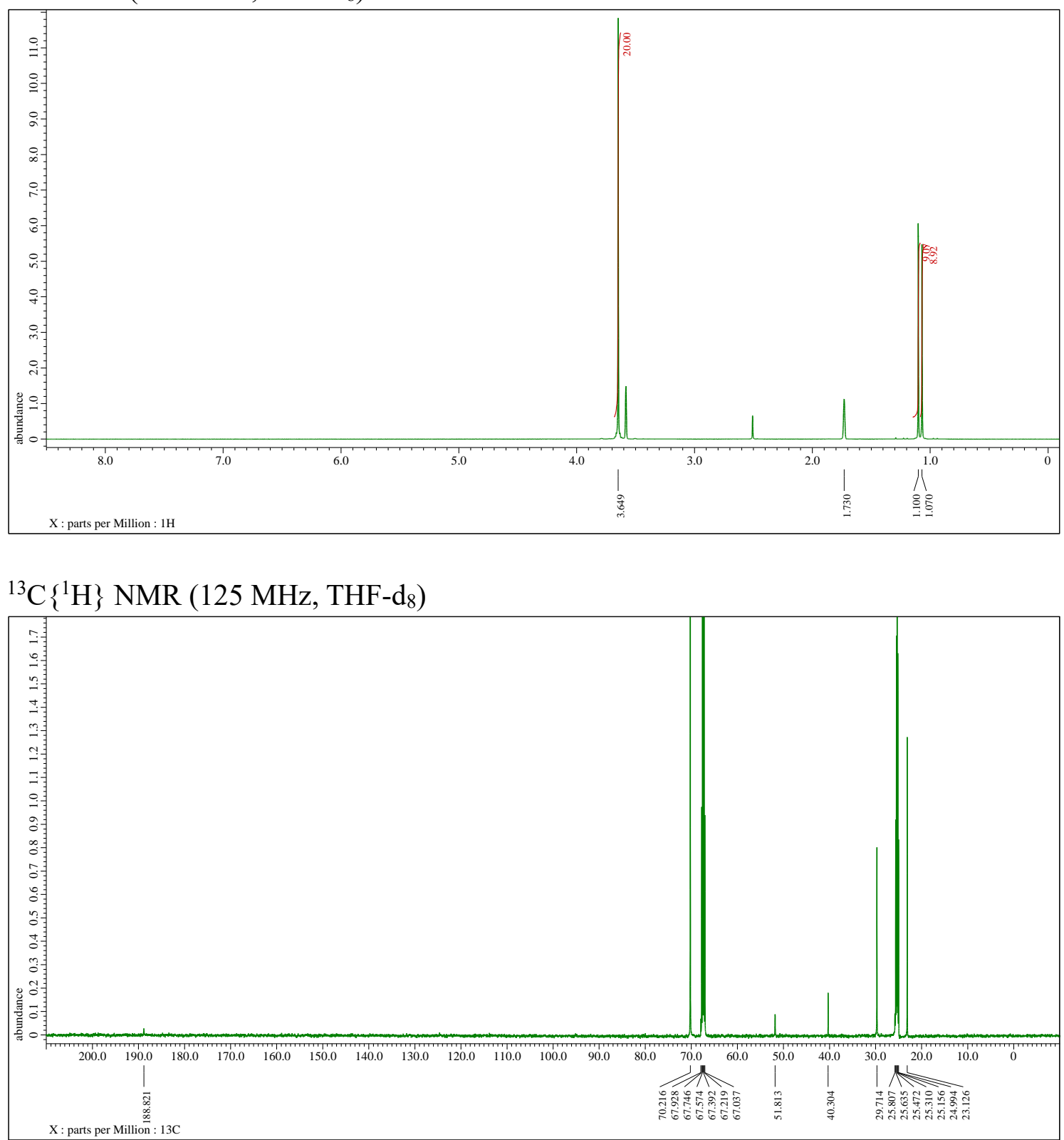
(S)-N-(tert-Butyl(ethoxy)- $\lambda^{4}$-sulfanylidene)pivalamide (5)

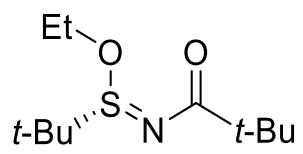

${ }^{1} \mathrm{H} \mathrm{NMR}\left(500 \mathrm{MHz}, \mathrm{CDCl}_{3}\right)$

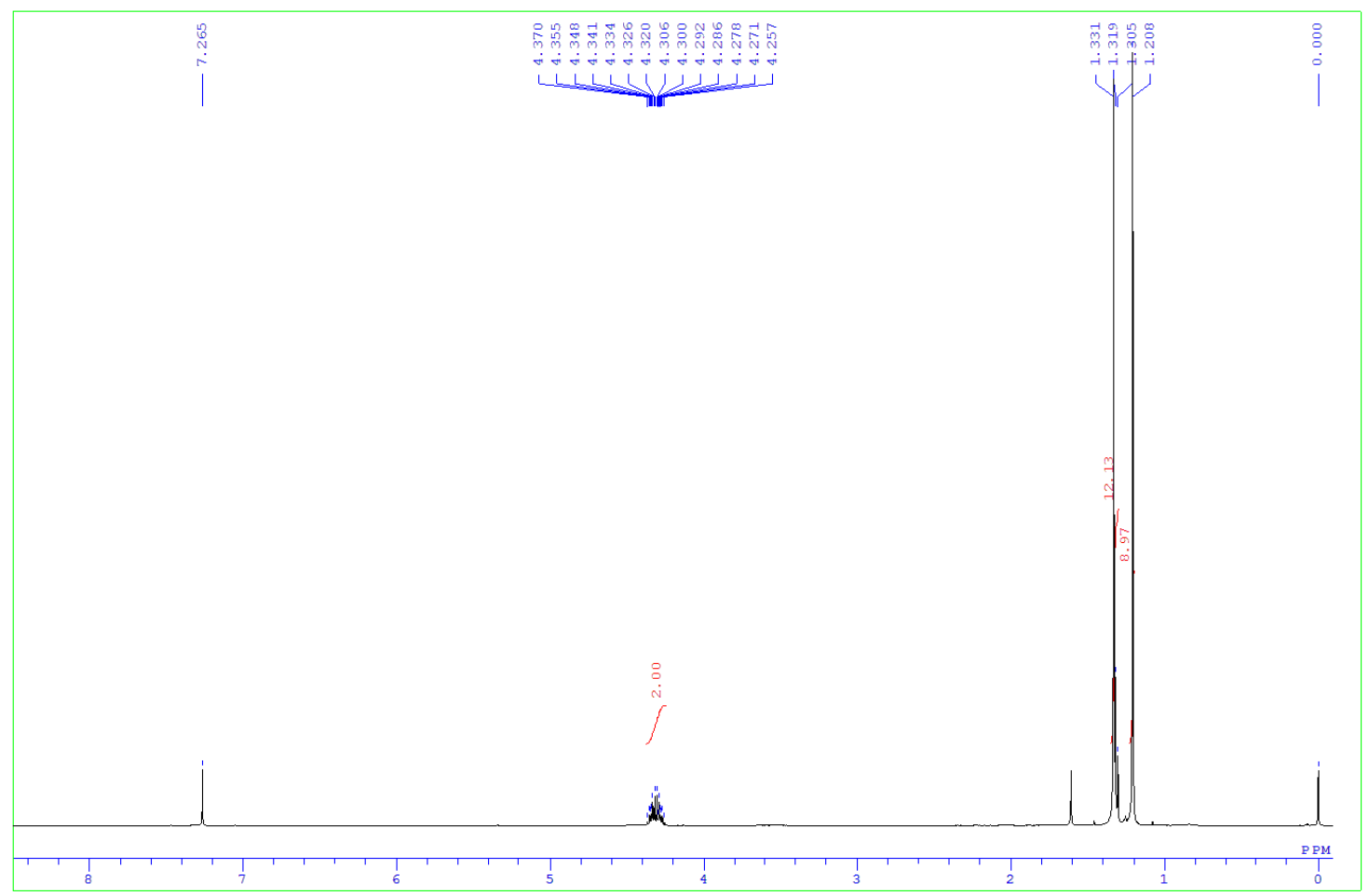



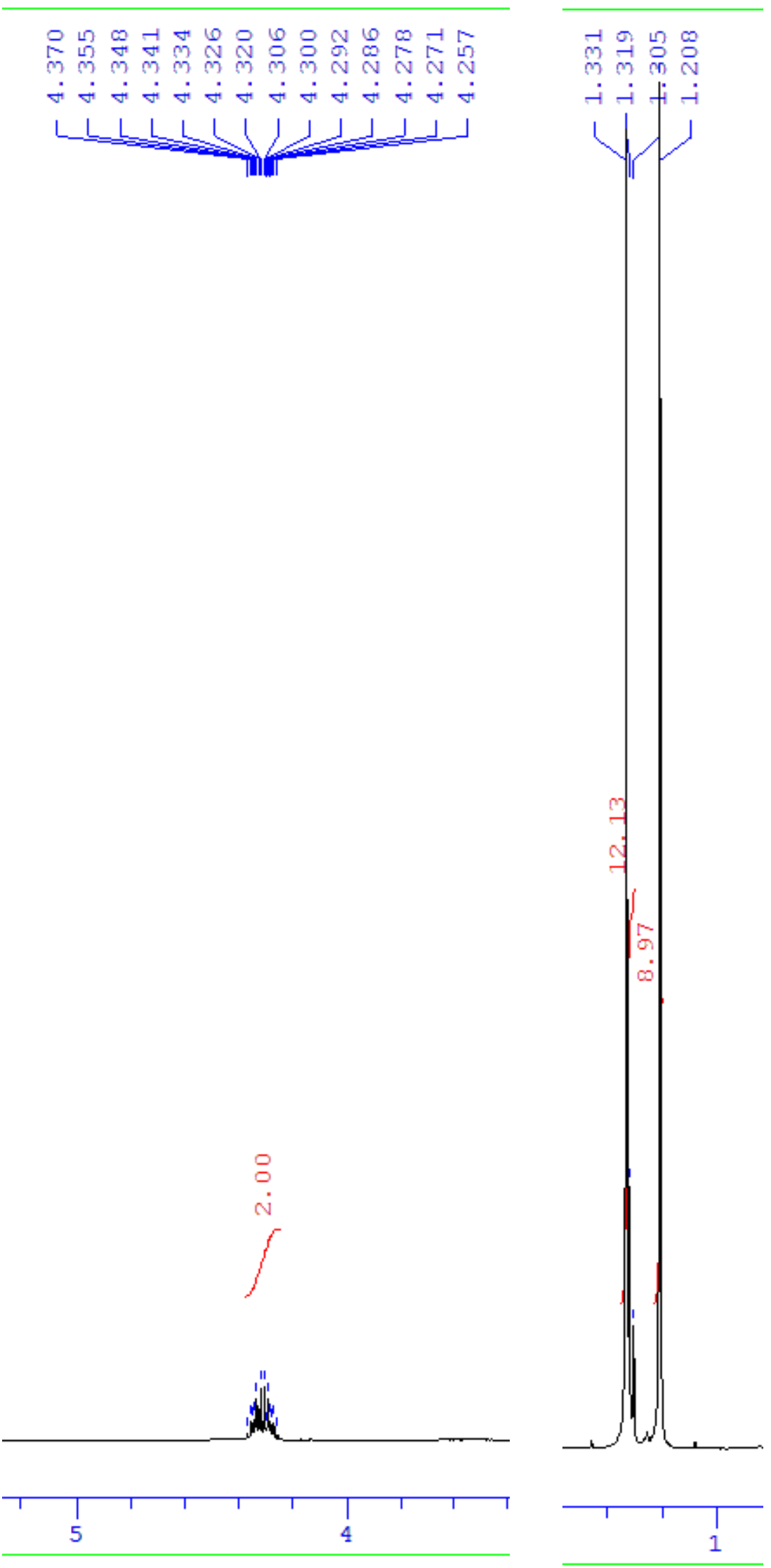
${ }^{13} \mathrm{C}\left\{{ }^{1} \mathrm{H}\right\}$ NMR $\left(125 \mathrm{MHz}, \mathrm{CDCl}_{3}\right)$

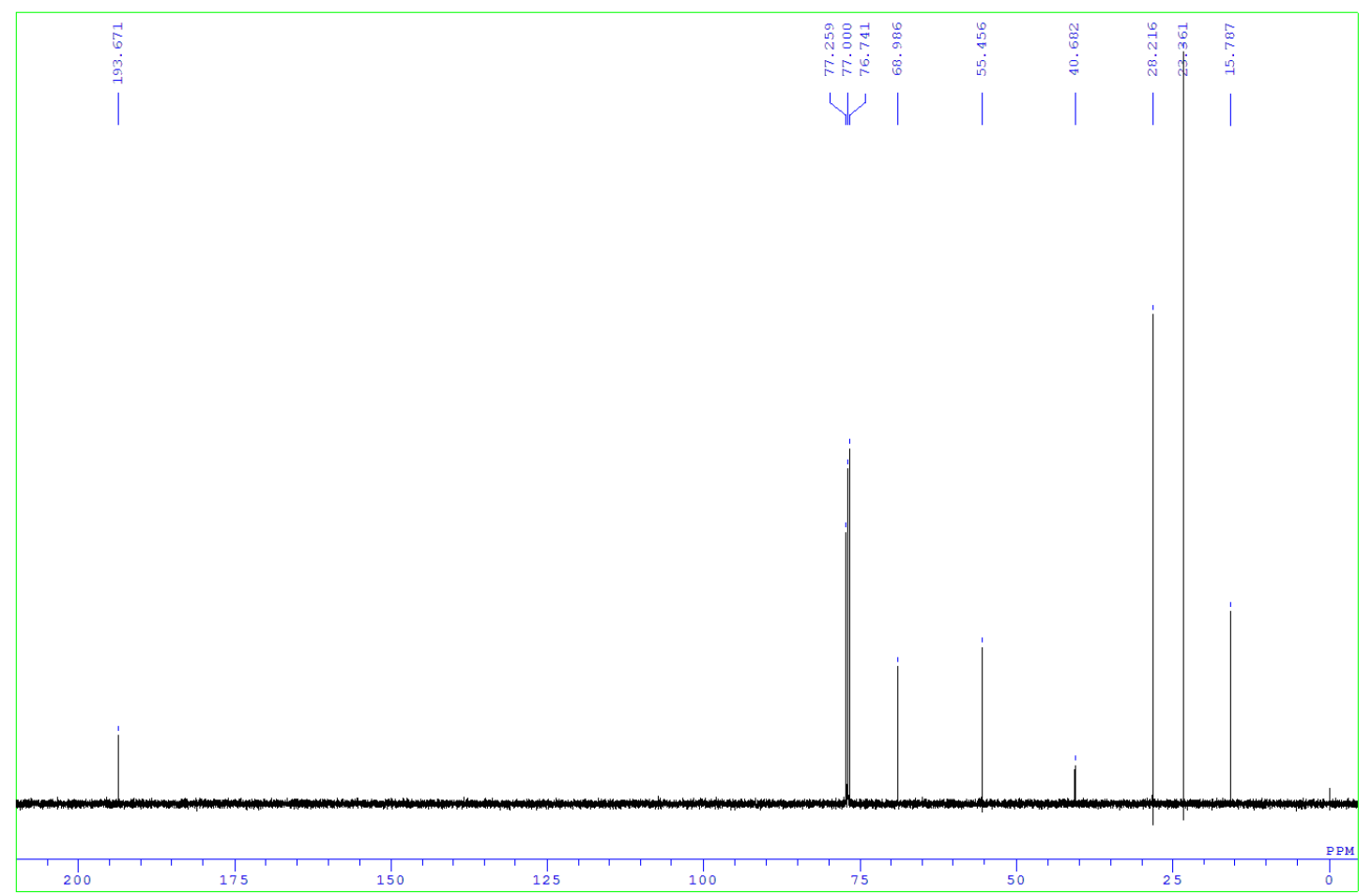


(R)- $N$-(tert-Butyl(ethyl)(oxo)- $\lambda^{6}$-sulfanylidene)pivalamide (2a)

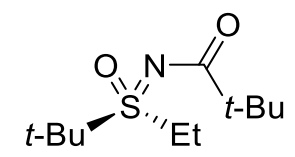

${ }^{1} \mathrm{H}$ NMR $\left(500 \mathrm{MHz}, \mathrm{CDCl}_{3}\right)$

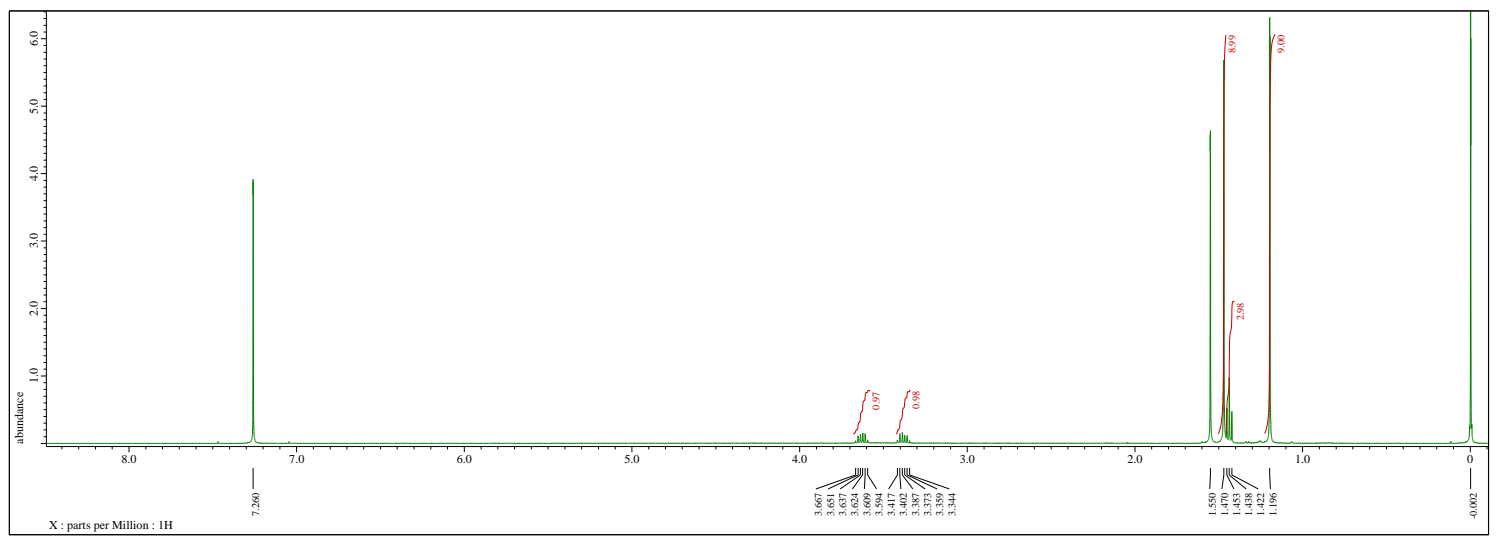


(R)-N-(tert-Butyl(methyl)(oxo)- $\lambda^{6}$-sulfanylidene)pivalamide (2b)

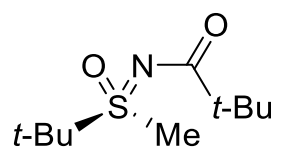

${ }^{1} \mathrm{H} \mathrm{NMR}\left(500 \mathrm{MHz}, \mathrm{CDCl}_{3}\right)$

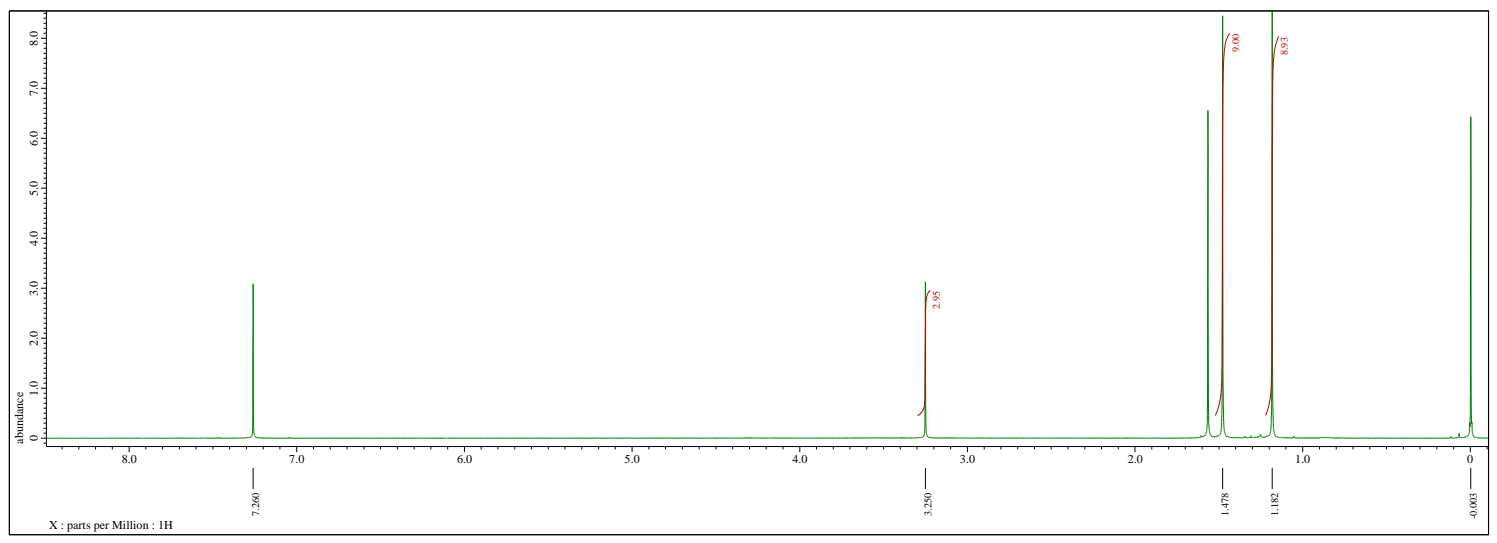


(R)- $N$-(tert-Butyl(butyl)(oxo)- $-\lambda^{6}$-sulfanylidene)pivalamide (2c)

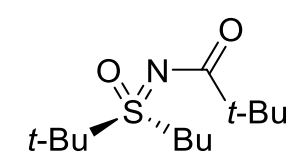

${ }^{1} \mathrm{H} \mathrm{NMR}\left(500 \mathrm{MHz}, \mathrm{CDCl}_{3}\right)$

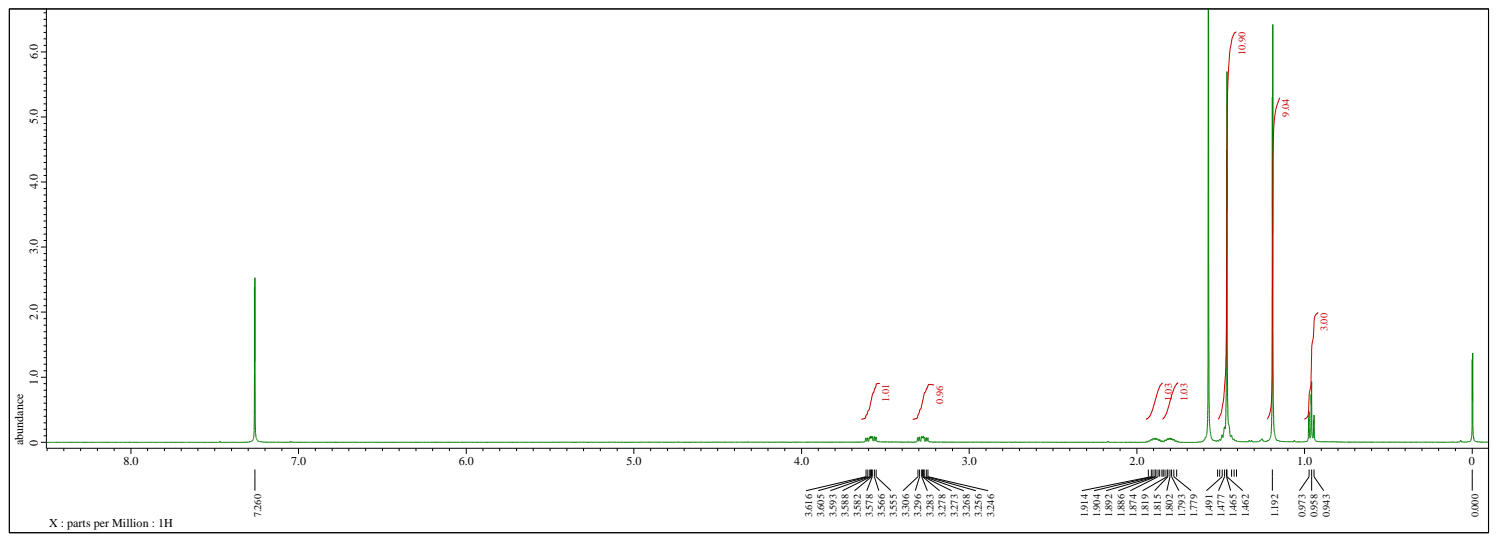


(R)- $N$-(tert-Butyl(hexyl)(oxo)- $\lambda^{6}$-sulfanylidene)pivalamide (2d)

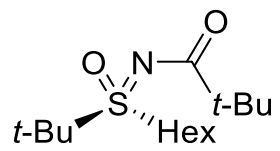

${ }^{1} \mathrm{H}$ NMR $\left(500 \mathrm{MHz}, \mathrm{CDCl}_{3}\right)$

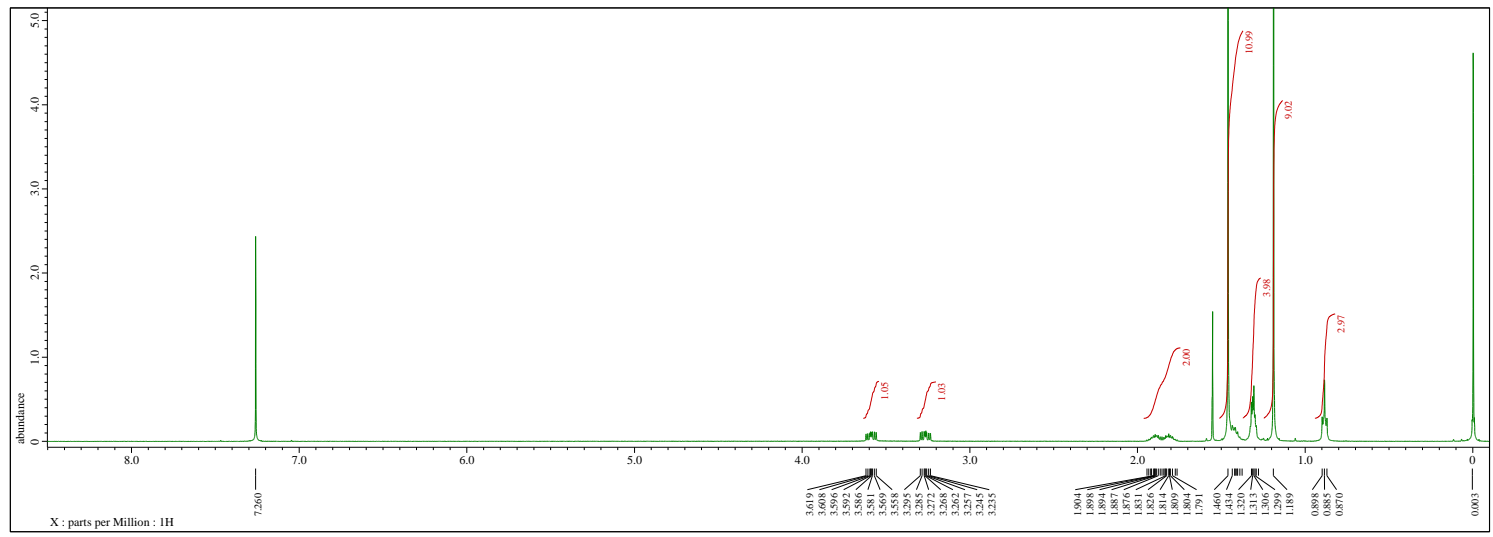


(R)-N-(tert-Butyl(isobutyl)(oxo)- $\lambda^{6}$-sulfanylidene)pivalamide (2e)

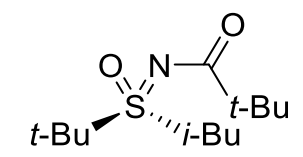

${ }^{1} \mathrm{H} \mathrm{NMR}\left(500 \mathrm{MHz}, \mathrm{CDCl}_{3}\right)$

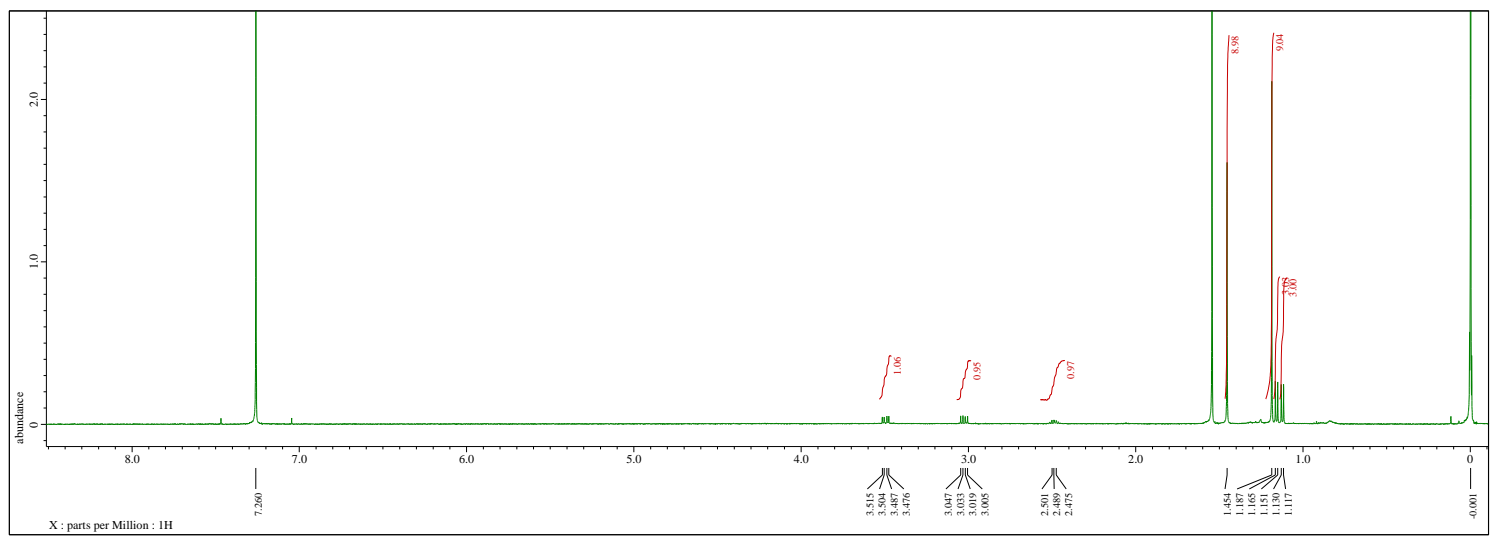


(R)-N-(tert-Butyl(oxo)(3-((triisopropylsilyl)oxy)propyl)- $\lambda^{6}-$ sulfanylidene)pivalamide (2f)

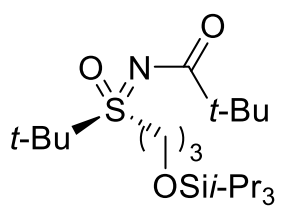

${ }^{1} \mathrm{H} \mathrm{NMR}\left(500 \mathrm{MHz}, \mathrm{CDCl}_{3}\right)$

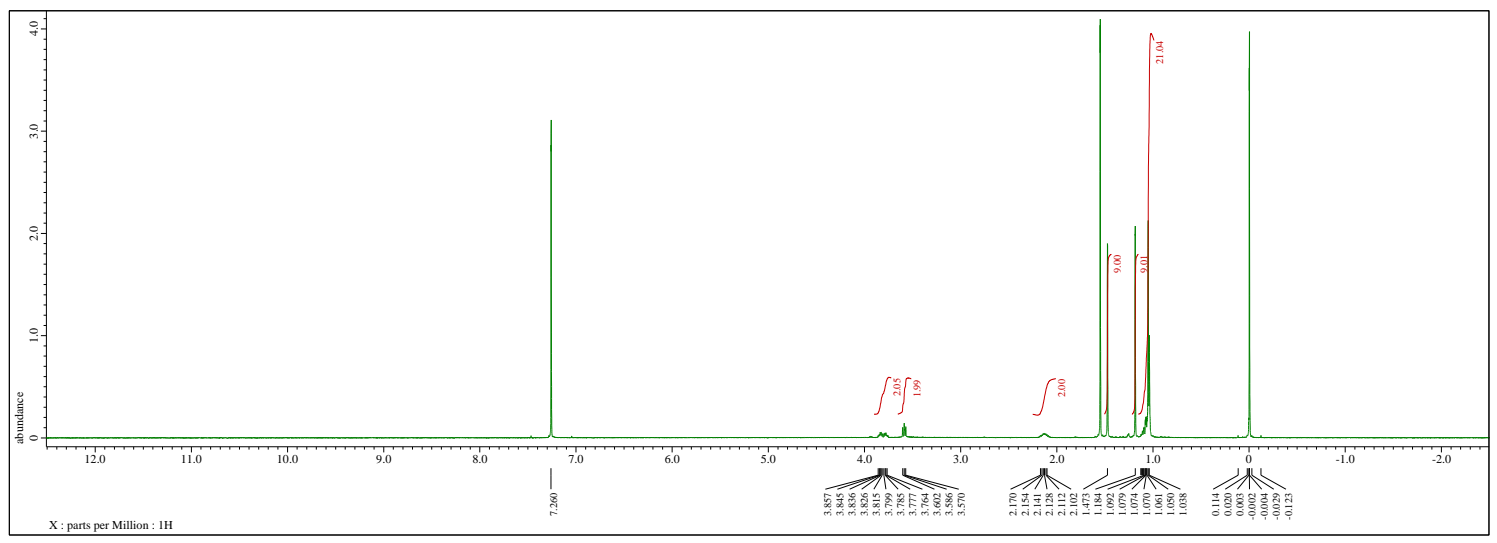


(R)-N-((4-(1,3-Dioxolan-2-yl)butyl)(tert-butyl)(oxo)- $\lambda^{6}$-sulfanylidene)pivalamide (2g)

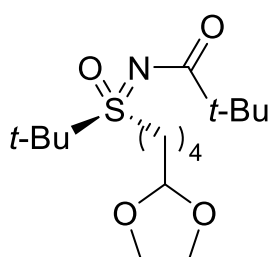

${ }^{1} \mathrm{H}$ NMR $\left(500 \mathrm{MHz}, \mathrm{CDCl}_{3}\right)$

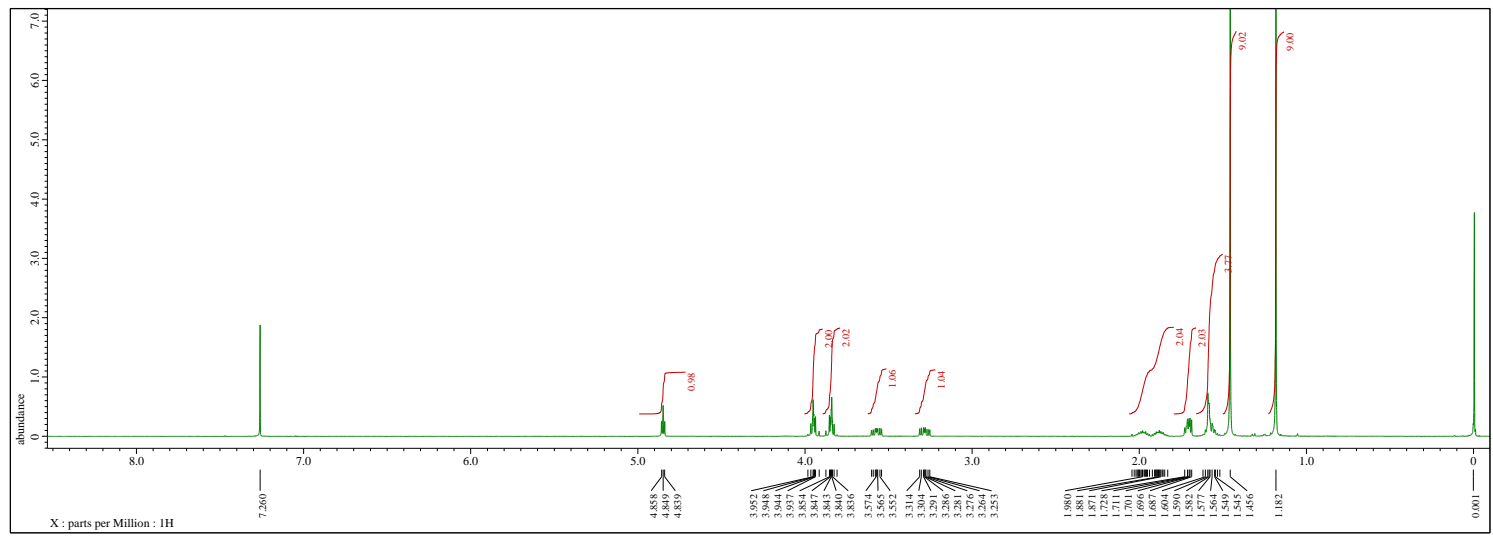


(R)-N-(tert-Butyl(5-iodopentyl)(oxo)- $\lambda^{6}$-sulfanylidene)pivalamide (2h)

${ }^{1} \mathrm{H}$ NMR $\left(500 \mathrm{MHz}, \mathrm{CDCl}_{3}\right)$
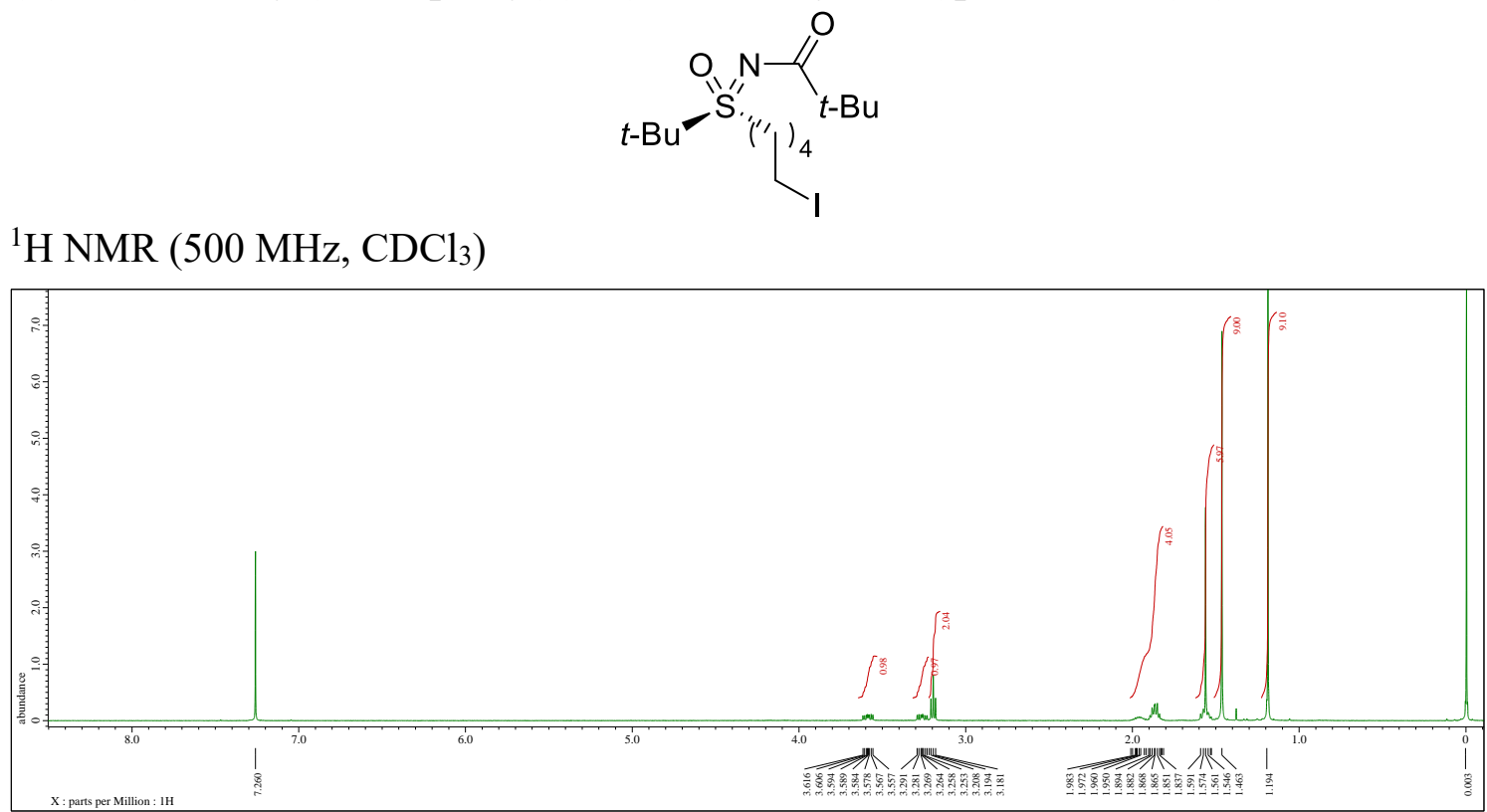
(R)- $N$-(Benzyl(tert-butyl)(oxo)- $\lambda^{6}$-sulfanylidene)pivalamide (2i)

${ }^{1} \mathrm{H}$ NMR $\left(500 \mathrm{MHz}, \mathrm{CDCl}_{3}\right)$
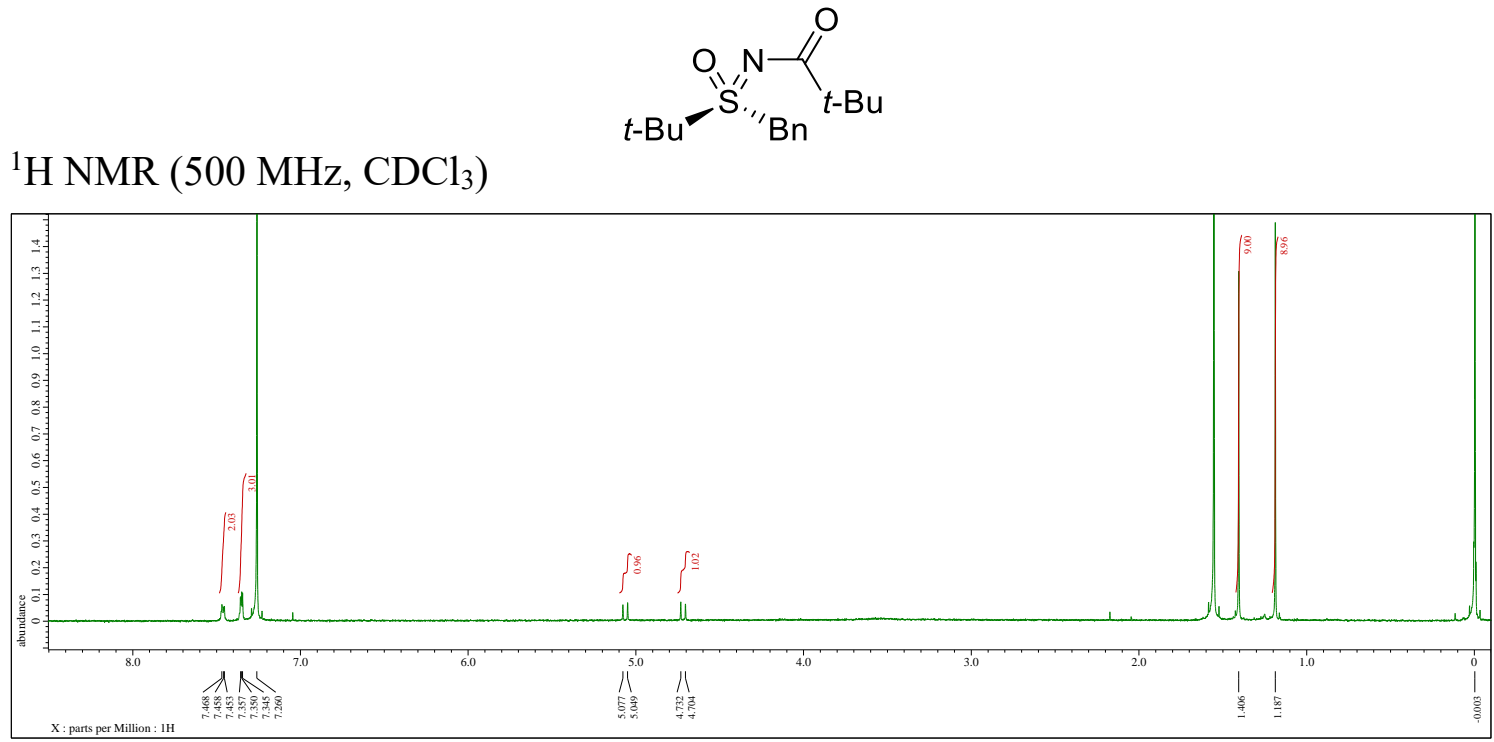
(R)- $N$-(tert-Butyl(allyl)(oxo)- $\lambda 6$-sulfanylidene)pivalamide (2j)

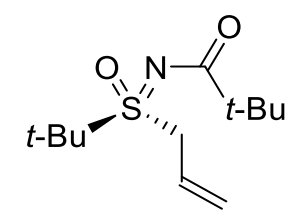

${ }^{1} \mathrm{H}$ NMR (500 MHz, $\left.\mathrm{CDCl}_{3}\right)$

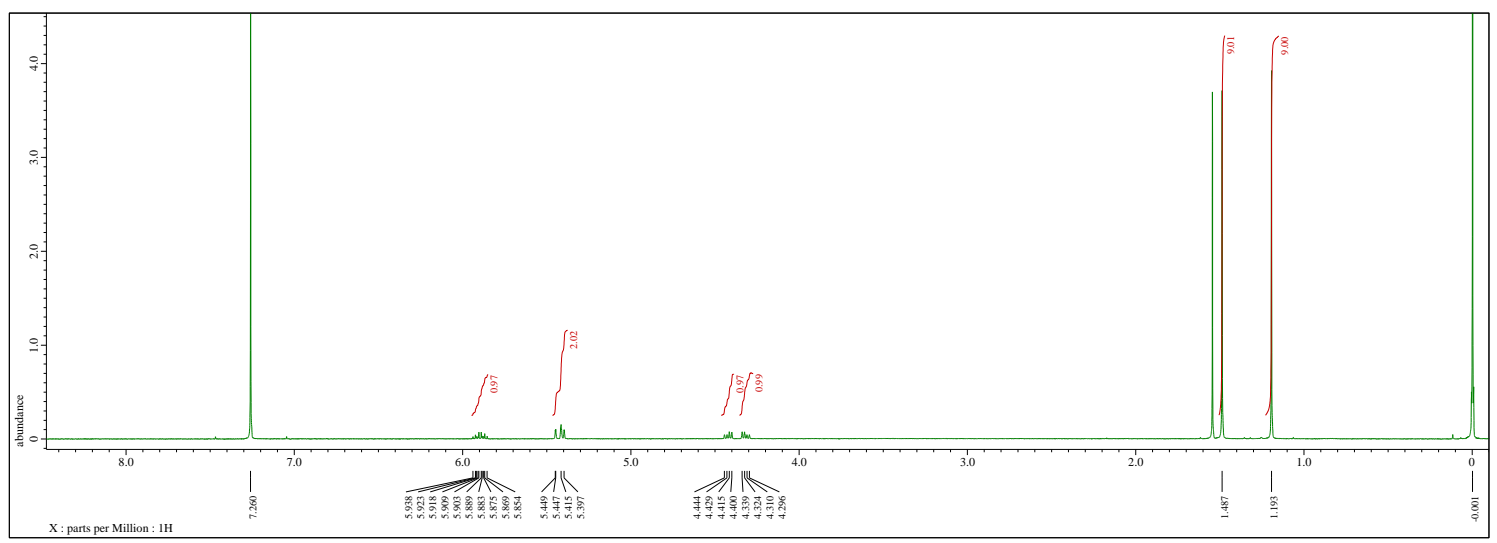


$(R, E)-N$-(But-2-en-1-yl(tert-butyl)(oxo)- $\lambda^{6}$-sulfanylidene)pivalamide (2k)

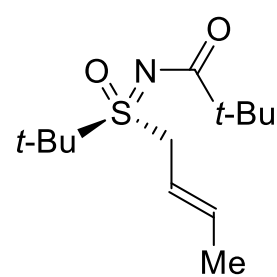

${ }^{1} \mathrm{H}$ NMR (500 MHz, $\mathrm{CDCl}_{3}$ ) (mixture of $E$ and $Z$ )
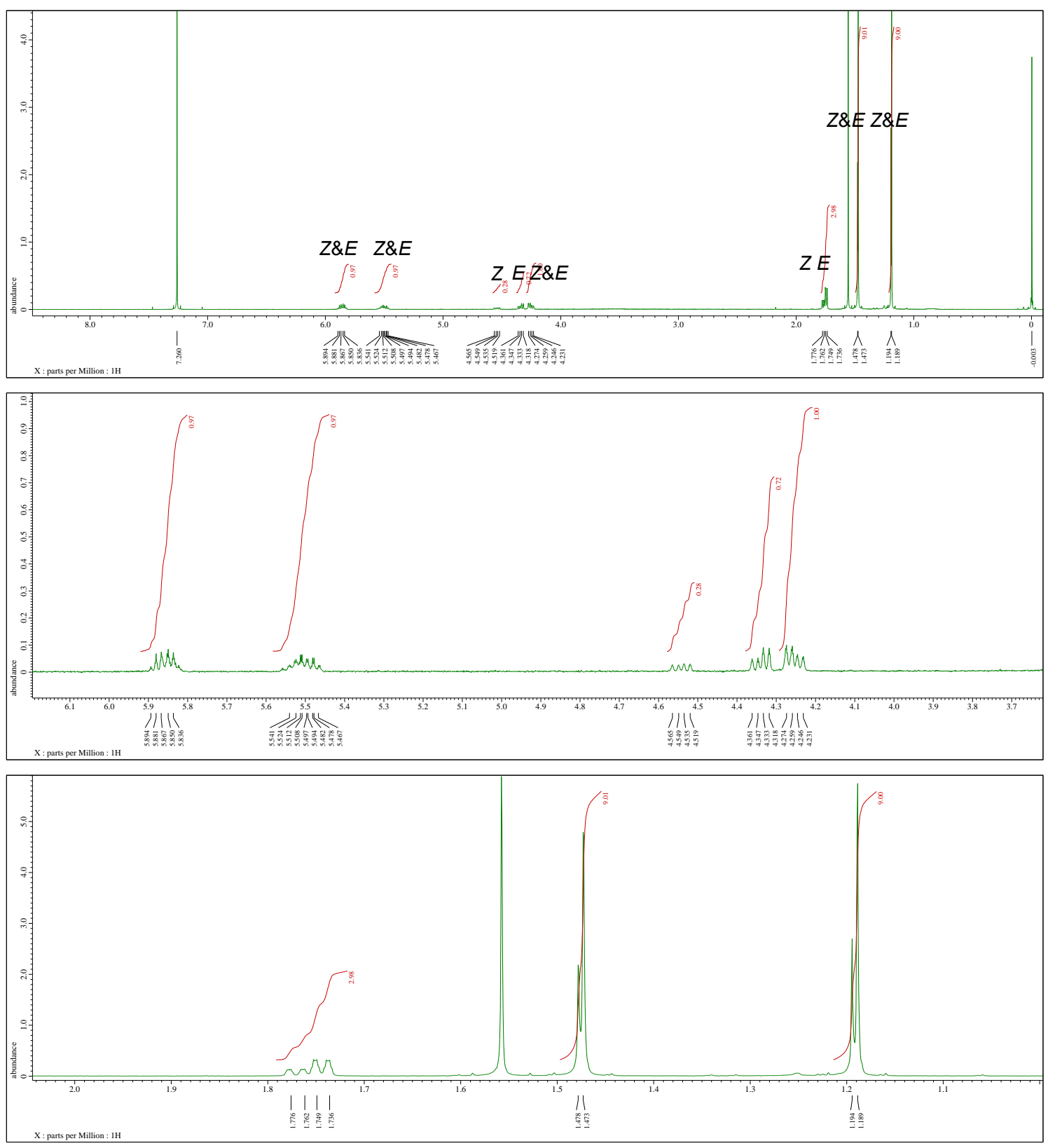
$(R, E)-N$-(3-Phenylprop-2-en-1-yl(tert-butyl)(oxo)- $-\lambda^{6}$-sulfanylidene)pivalamide (2l)

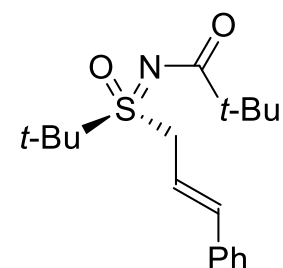

${ }^{1} \mathrm{H} \mathrm{NMR}\left(500 \mathrm{MHz}, \mathrm{CDCl}_{3}\right)$
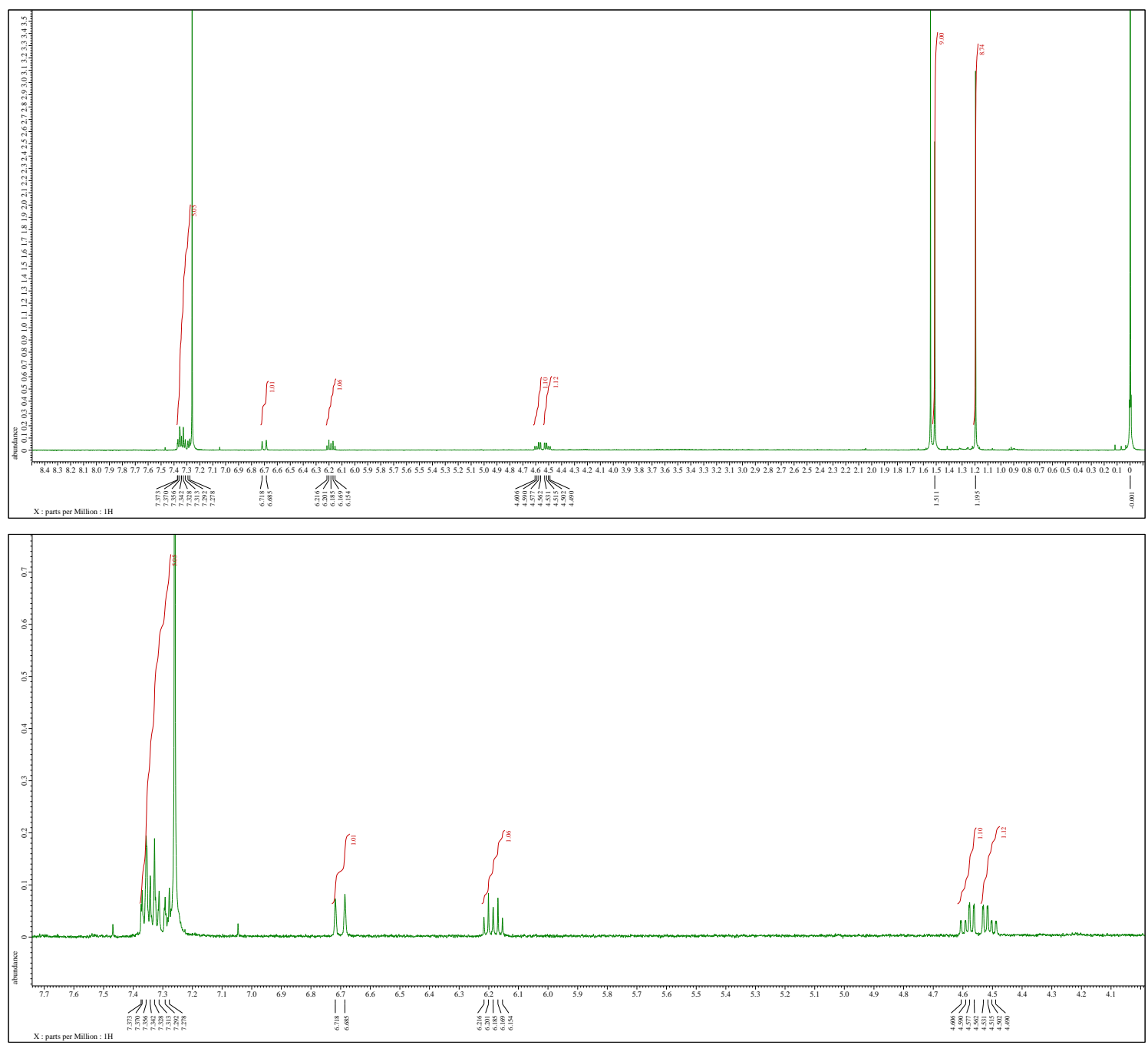


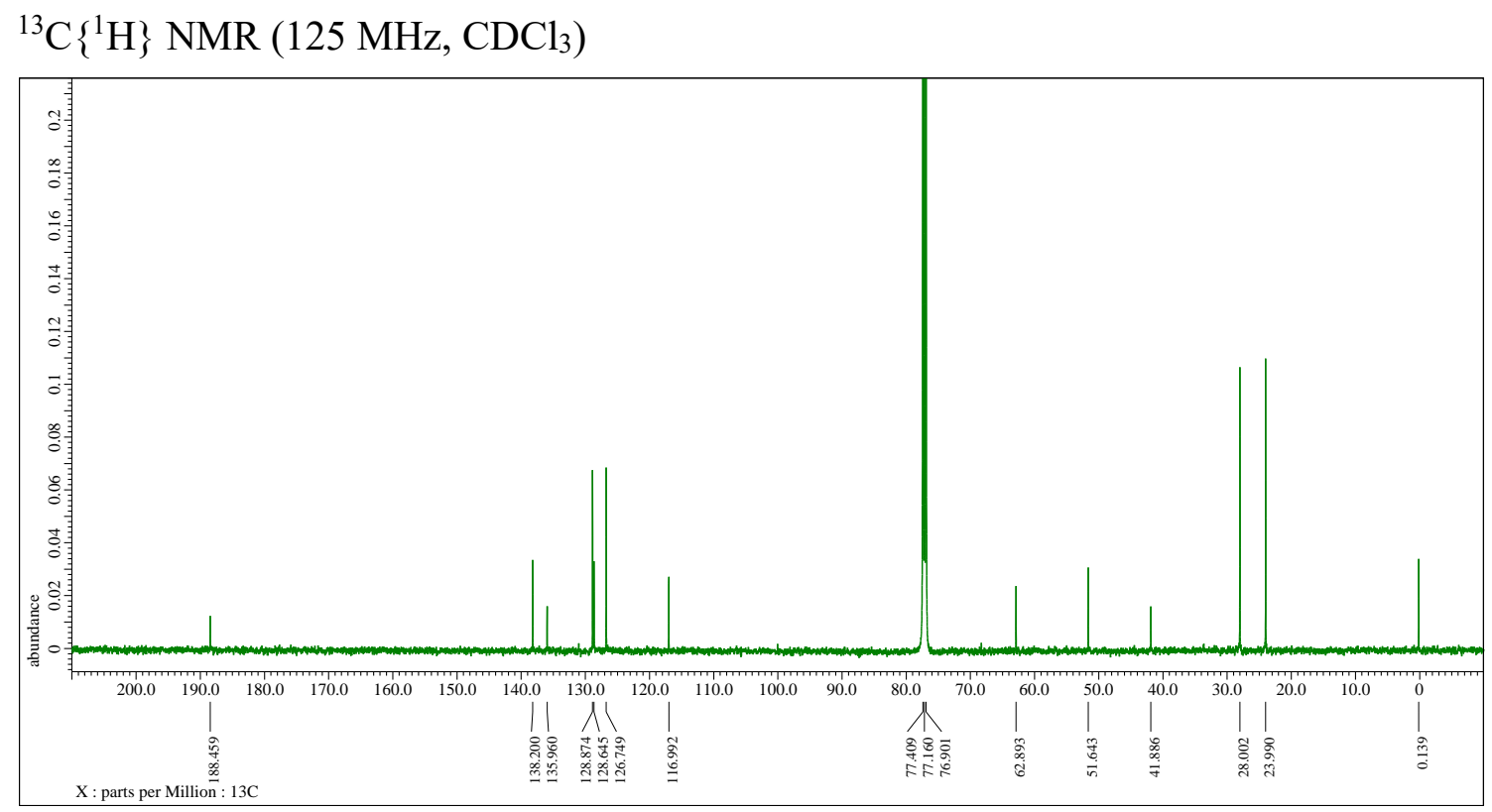


(R)- $N$-(2-Methylprop-2-en-1-yl(tert-butyl)(oxo)- $\lambda^{6}$-sulfanylidene)pivalamide (2m)

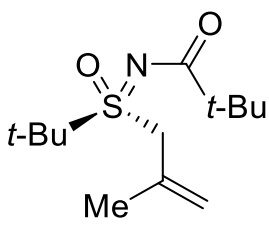

${ }^{1} \mathrm{H}$ NMR $\left(500 \mathrm{MHz}, \mathrm{CDCl}_{3}\right)$
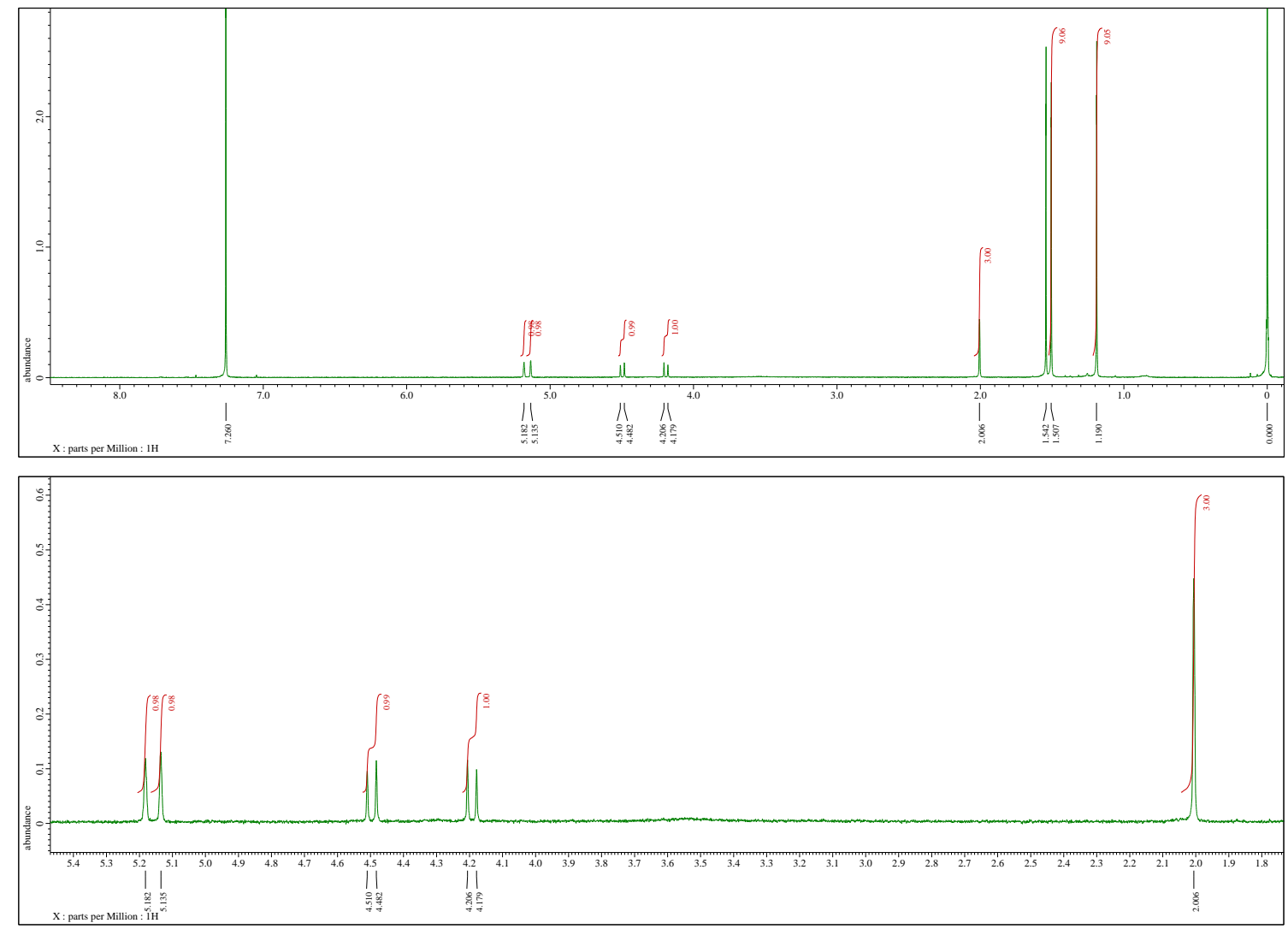
${ }^{13} \mathrm{C}\left\{{ }^{1} \mathrm{H}\right\}$ NMR $\left(125 \mathrm{MHz}, \mathrm{CDCl}_{3}\right)$

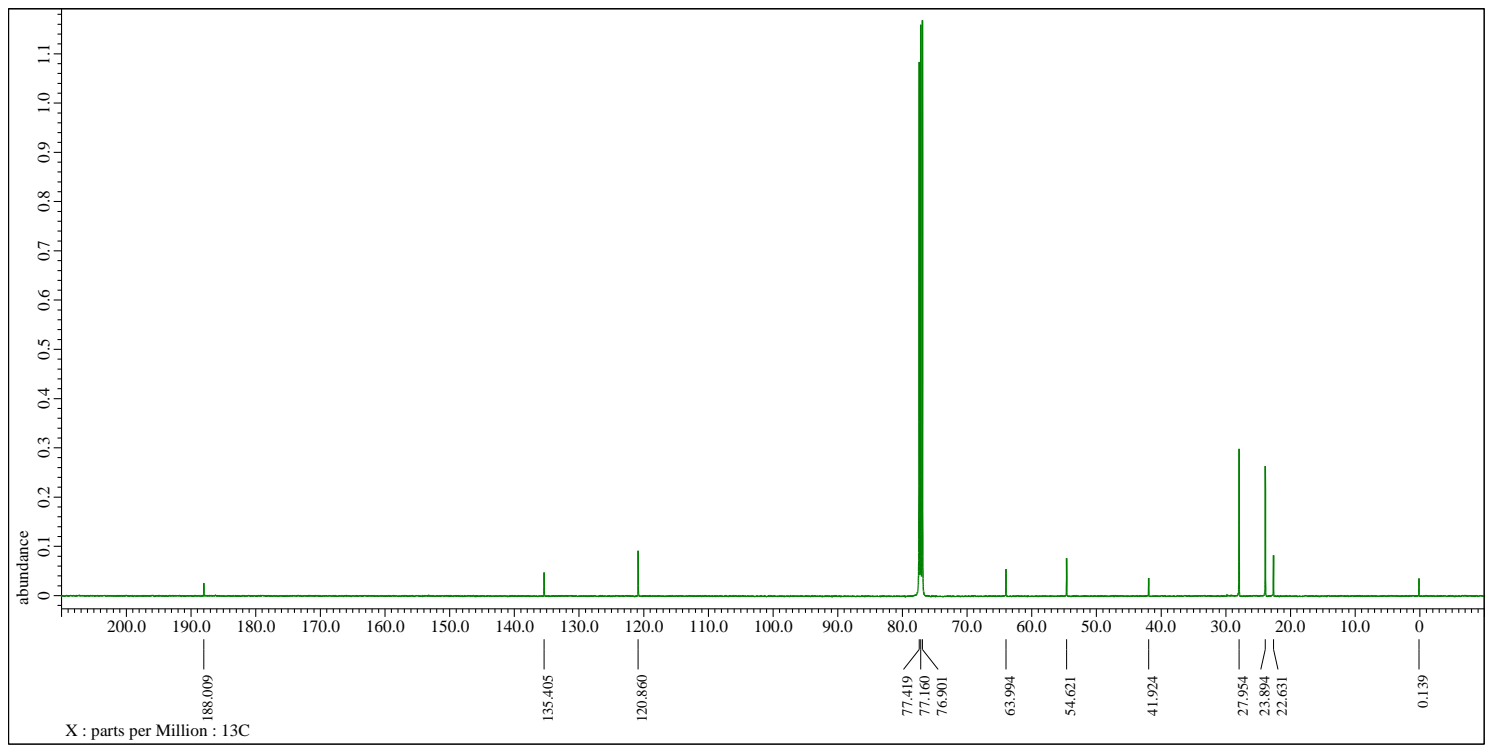


$(R, E)-N$-(2-Methylbut-2-en-1-yl(tert-butyl)(oxo)- $\lambda^{6}$-sulfanylidene)pivalamide (2n)

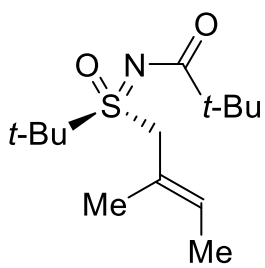

${ }^{1} \mathrm{H} \mathrm{NMR}\left(500 \mathrm{MHz}, \mathrm{CDCl}_{3}\right)$
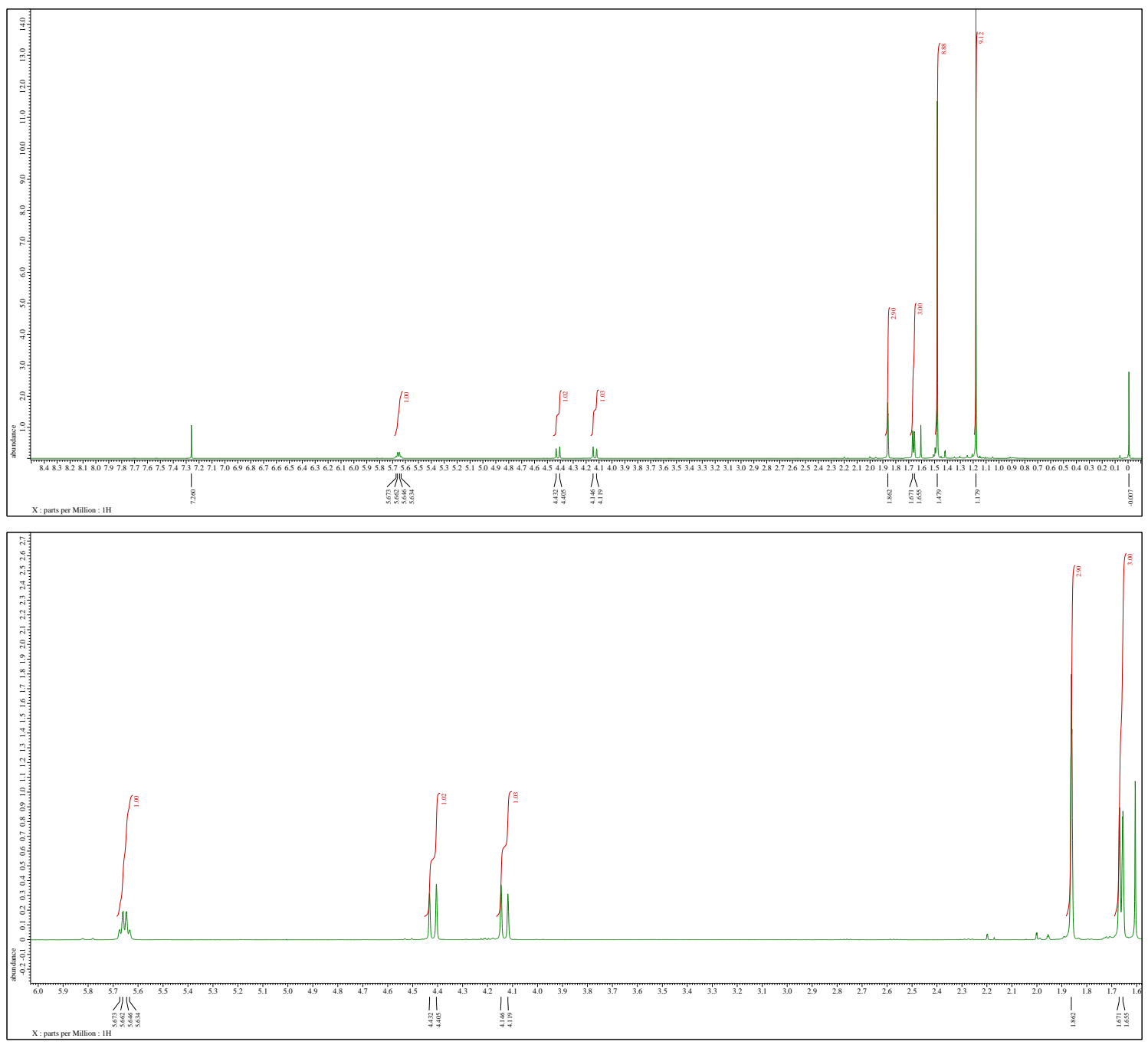
${ }^{13} \mathrm{C}\left\{{ }^{1} \mathrm{H}\right\} \mathrm{NMR}\left(125 \mathrm{MHz}, \mathrm{CDCl}_{3}\right)$

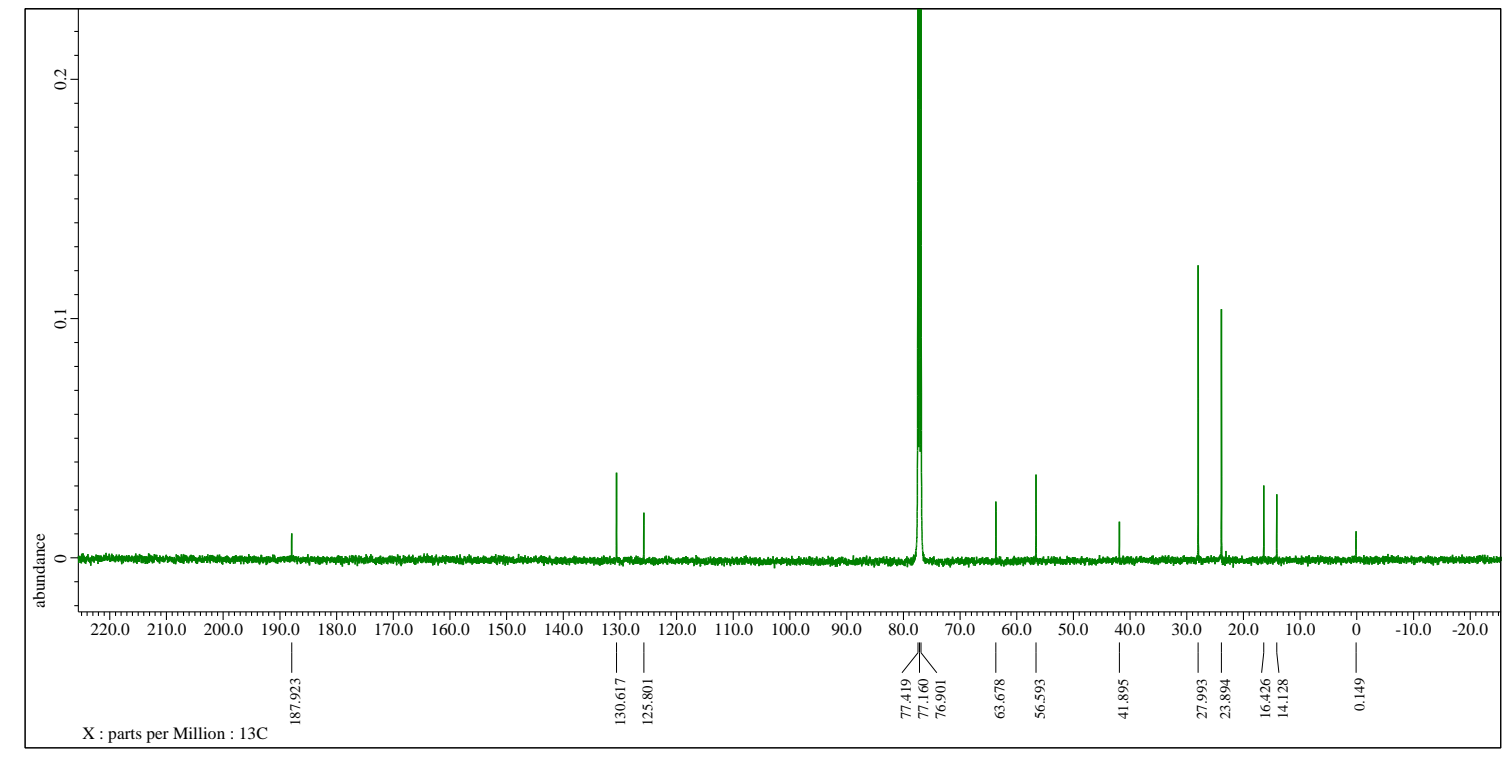


(R)- $N$-(But-2-yn-1-yl(tert-butyl)(oxo)- $\lambda^{6}$-sulfanylidene)pivalamide (2o)

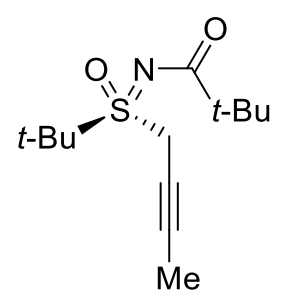

${ }^{1} \mathrm{H} \mathrm{NMR}\left(500 \mathrm{MHz}, \mathrm{CDCl}_{3}\right)$ (mixture of $\mathbf{2 o}$ and $\left.\mathbf{2 p}\right)$
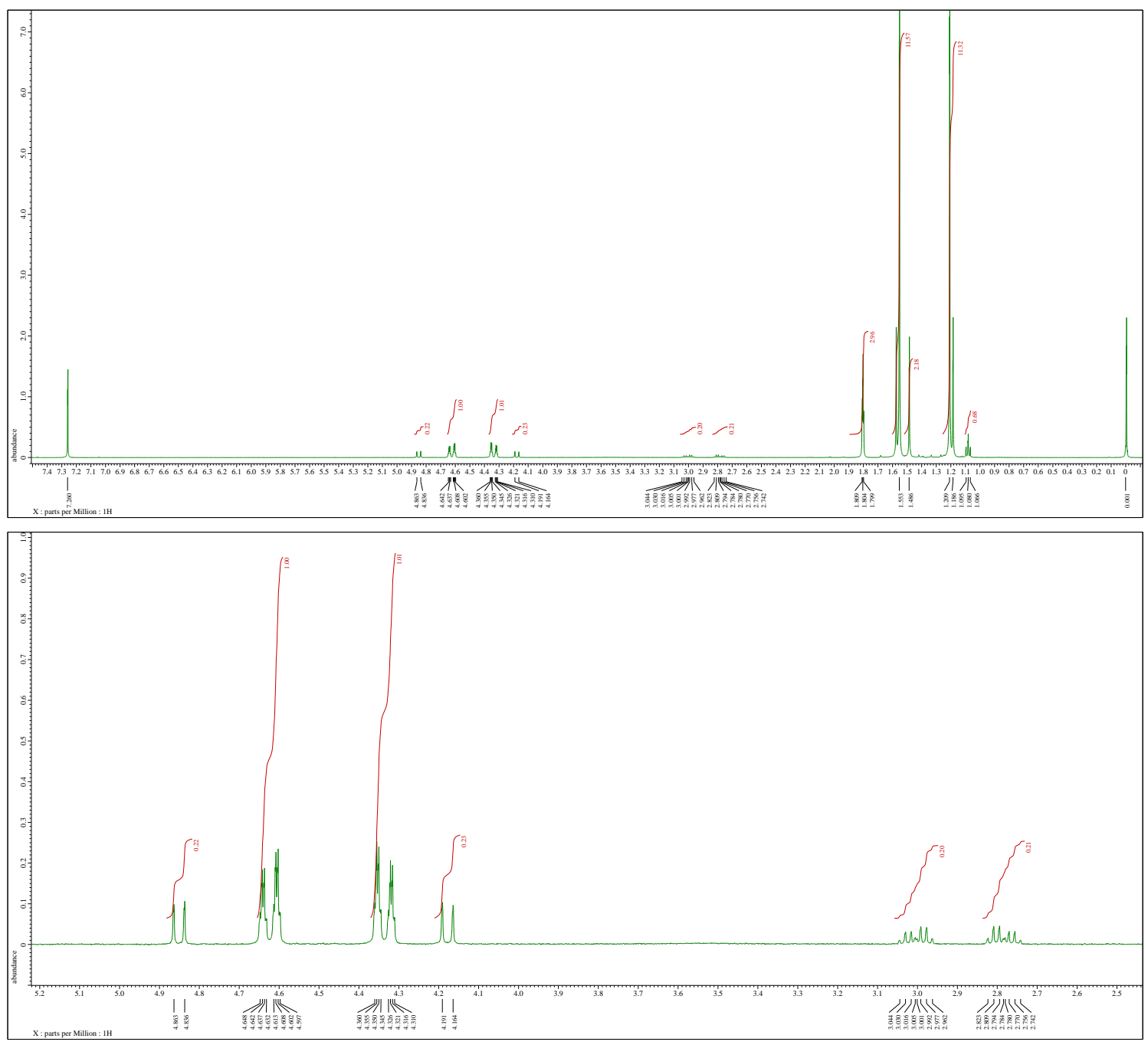


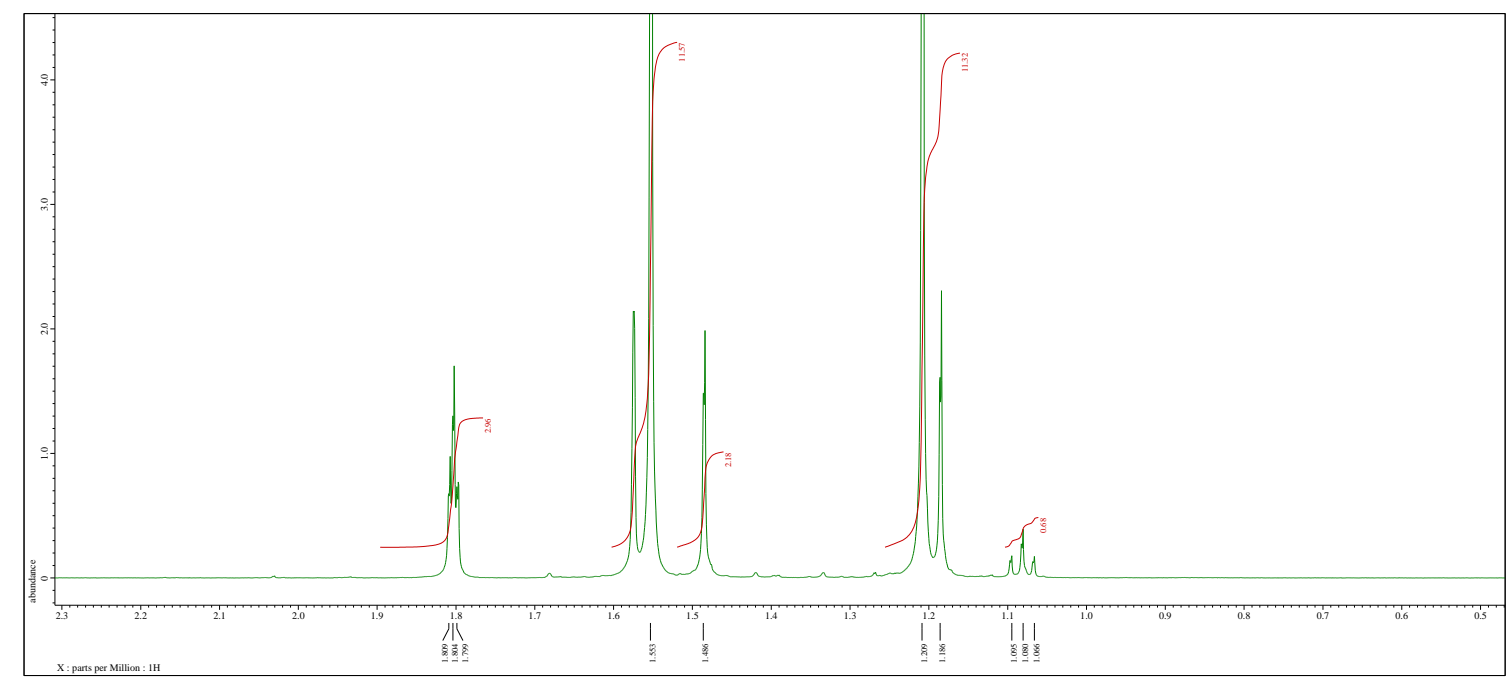


(R)-N-(tert-Butyl(2-oxobutyl)(oxo)- $\lambda^{6}$-sulfanylidene)pivalamide (2p)

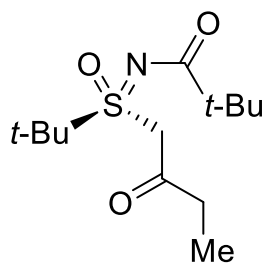

${ }^{1} \mathrm{H} \mathrm{NMR}\left(500 \mathrm{MHz}, \mathrm{CDCl}_{3}\right)$
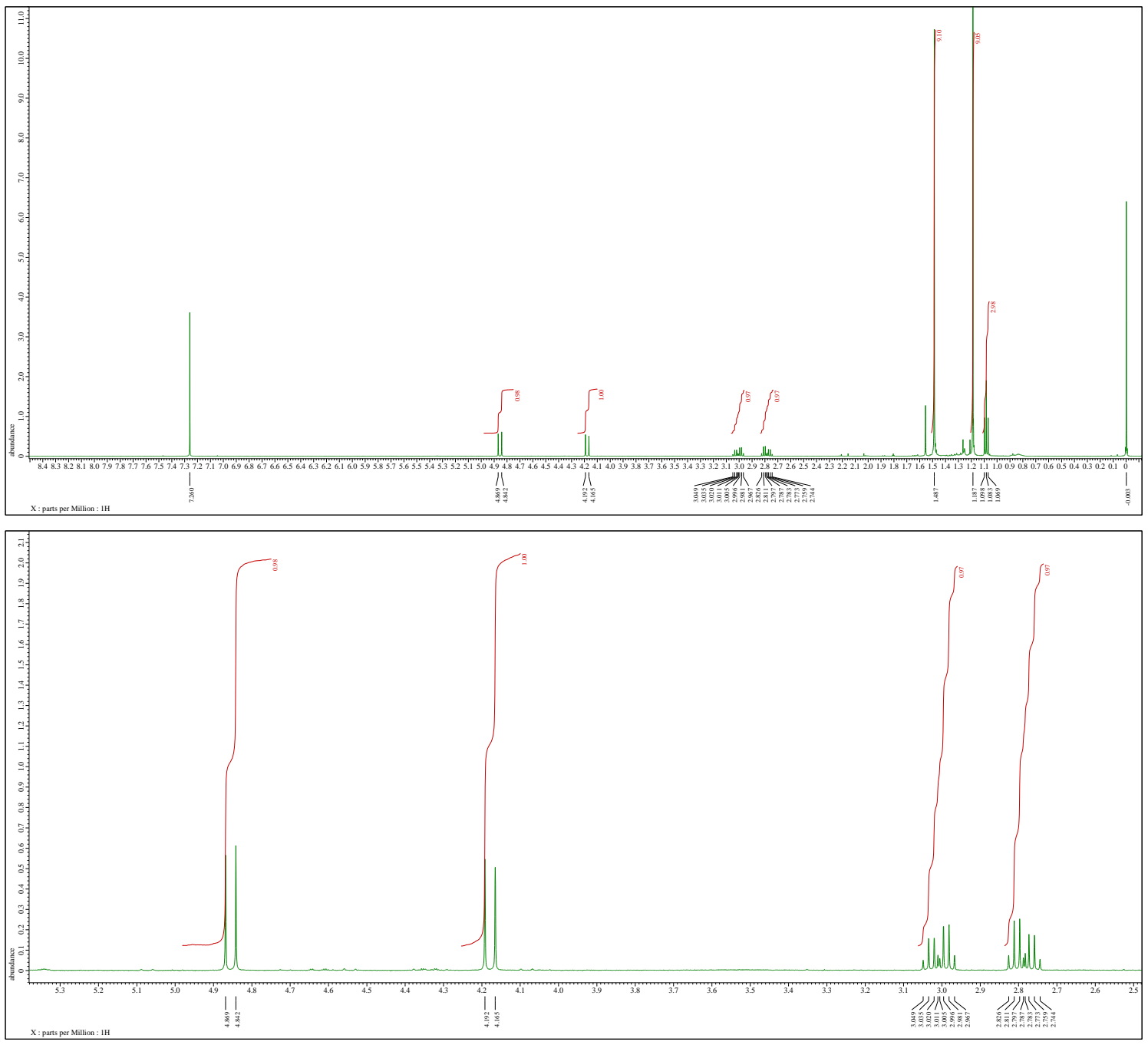


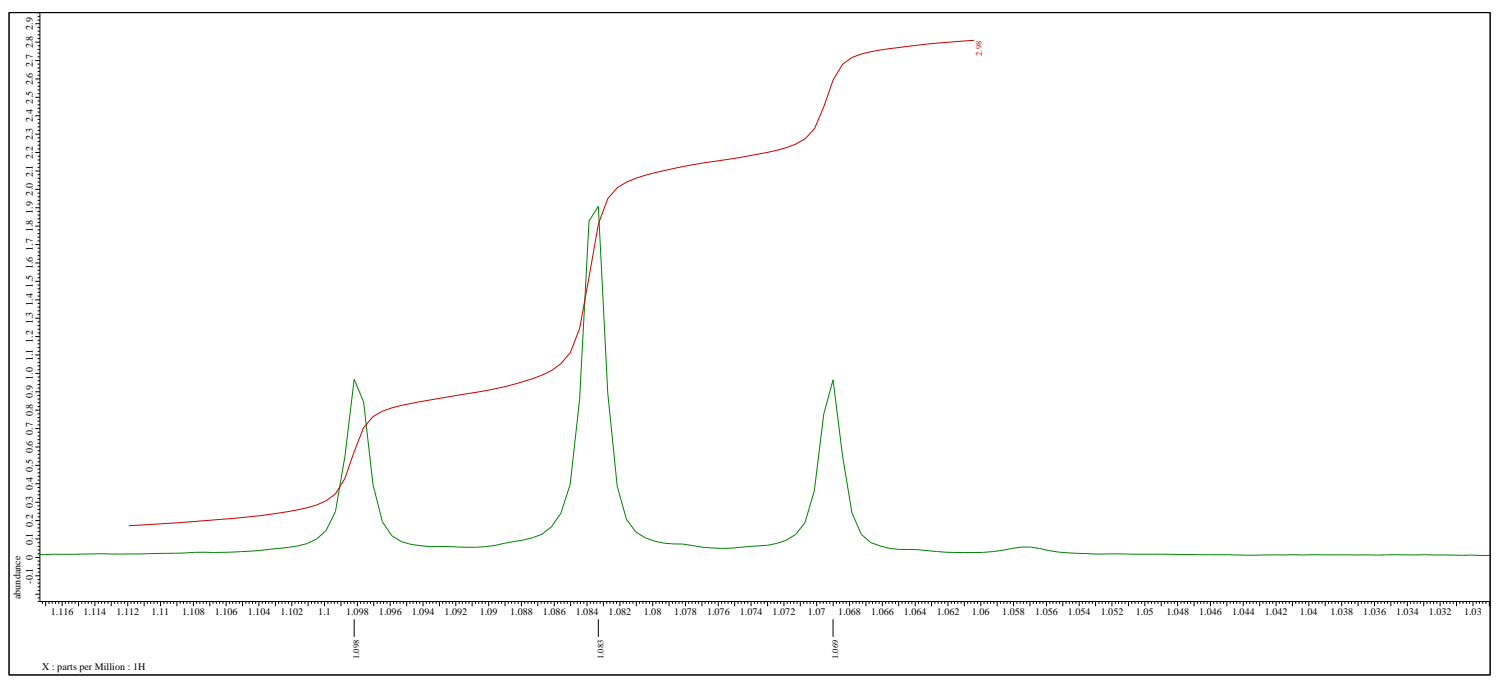

${ }^{13} \mathrm{C}\left\{{ }^{1} \mathrm{H}\right\}$ NMR $\left(125 \mathrm{MHz}, \mathrm{CDCl}_{3}\right)$

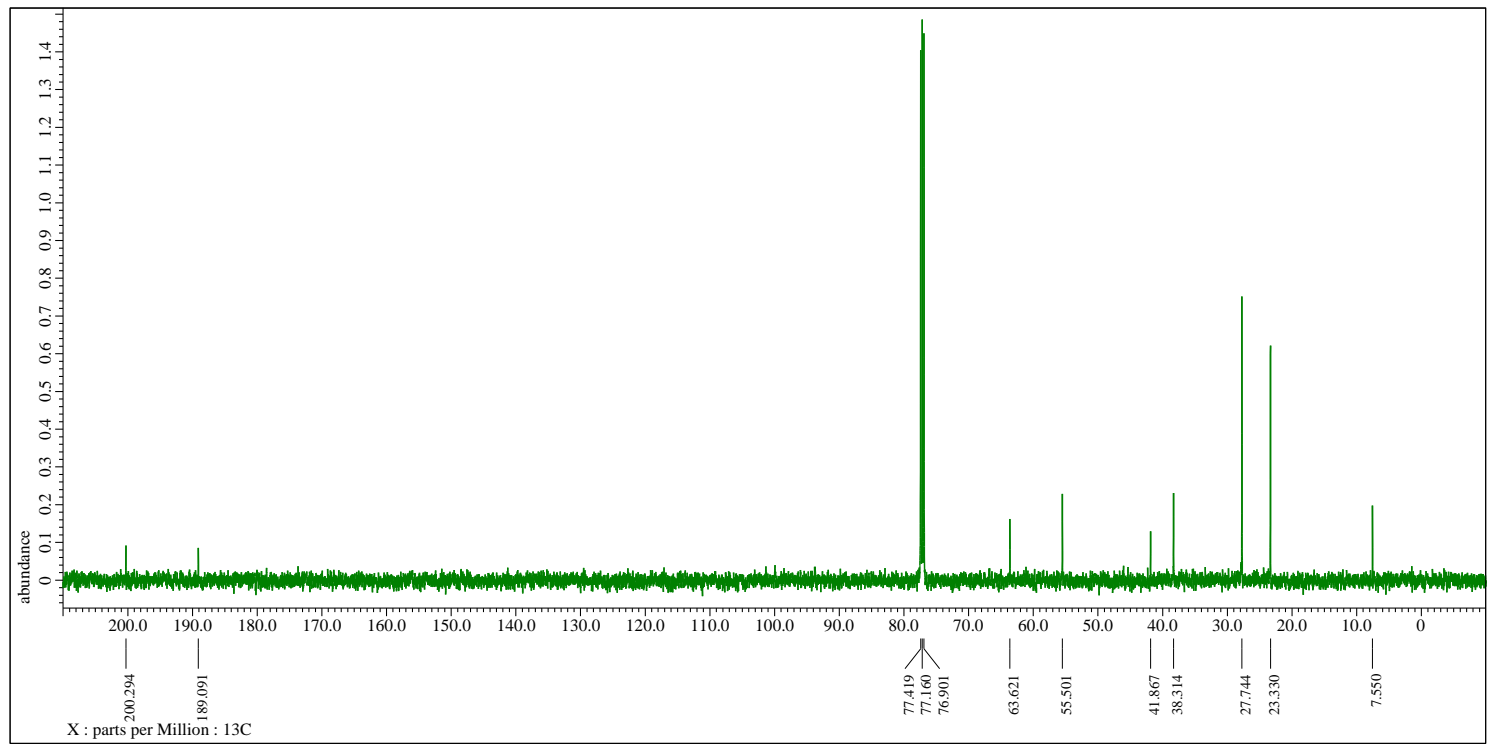


(R)- $N$-(tert-Butyl(2-oxo-3-phenylpropyl)(oxo)- $\lambda^{6}$-sulfanylidene)pivalamide (2q)

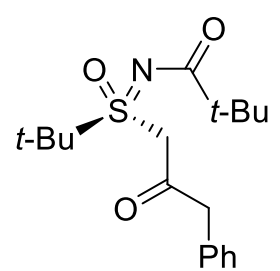

${ }^{1} \mathrm{H}$ NMR $\left(500 \mathrm{MHz}, \mathrm{CDCl}_{3}\right)$
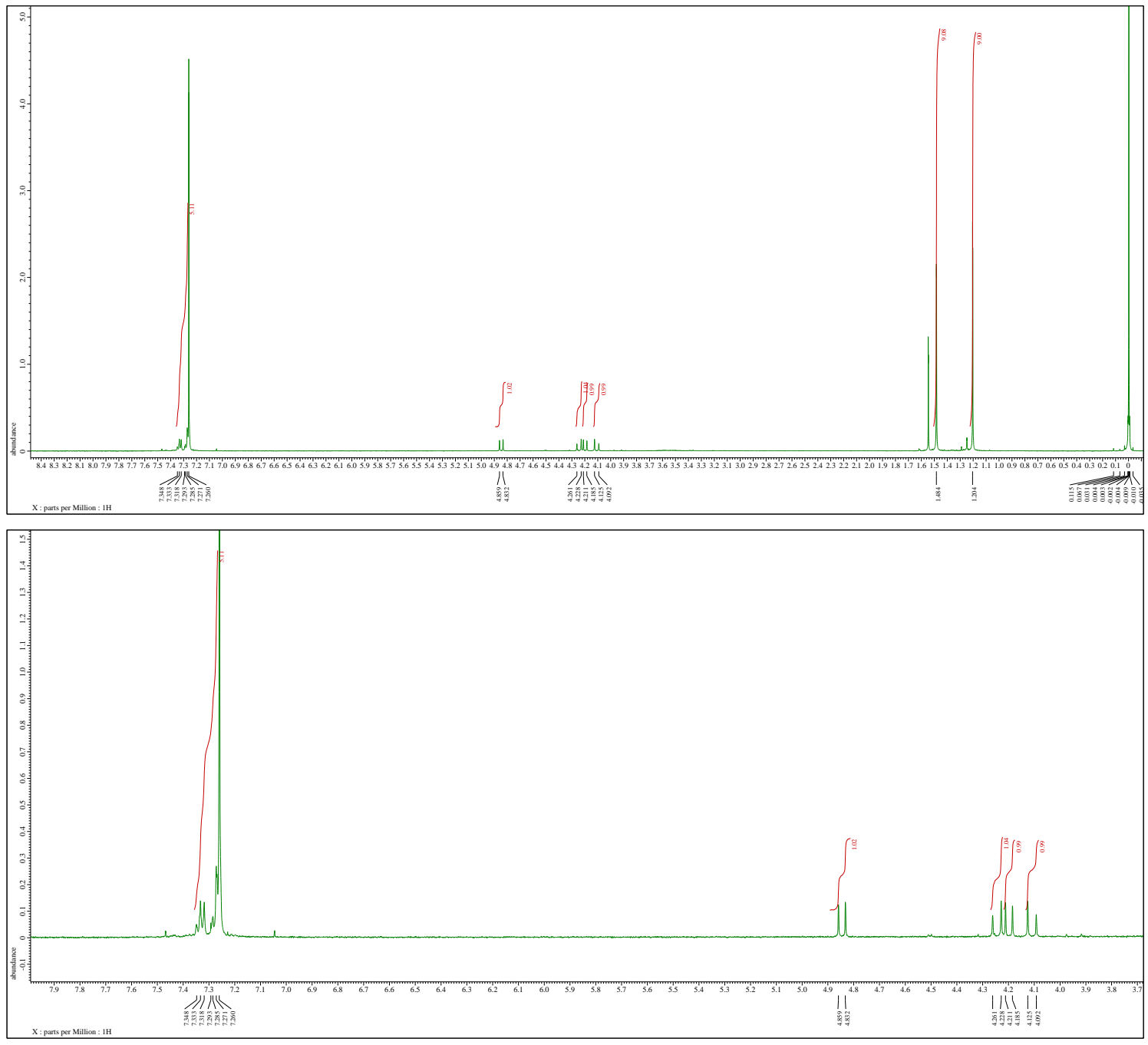
${ }^{13} \mathrm{C}\left\{{ }^{1} \mathrm{H}\right\}$ NMR $\left(125 \mathrm{MHz}, \mathrm{CD}_{3} \mathrm{CN}\right)$

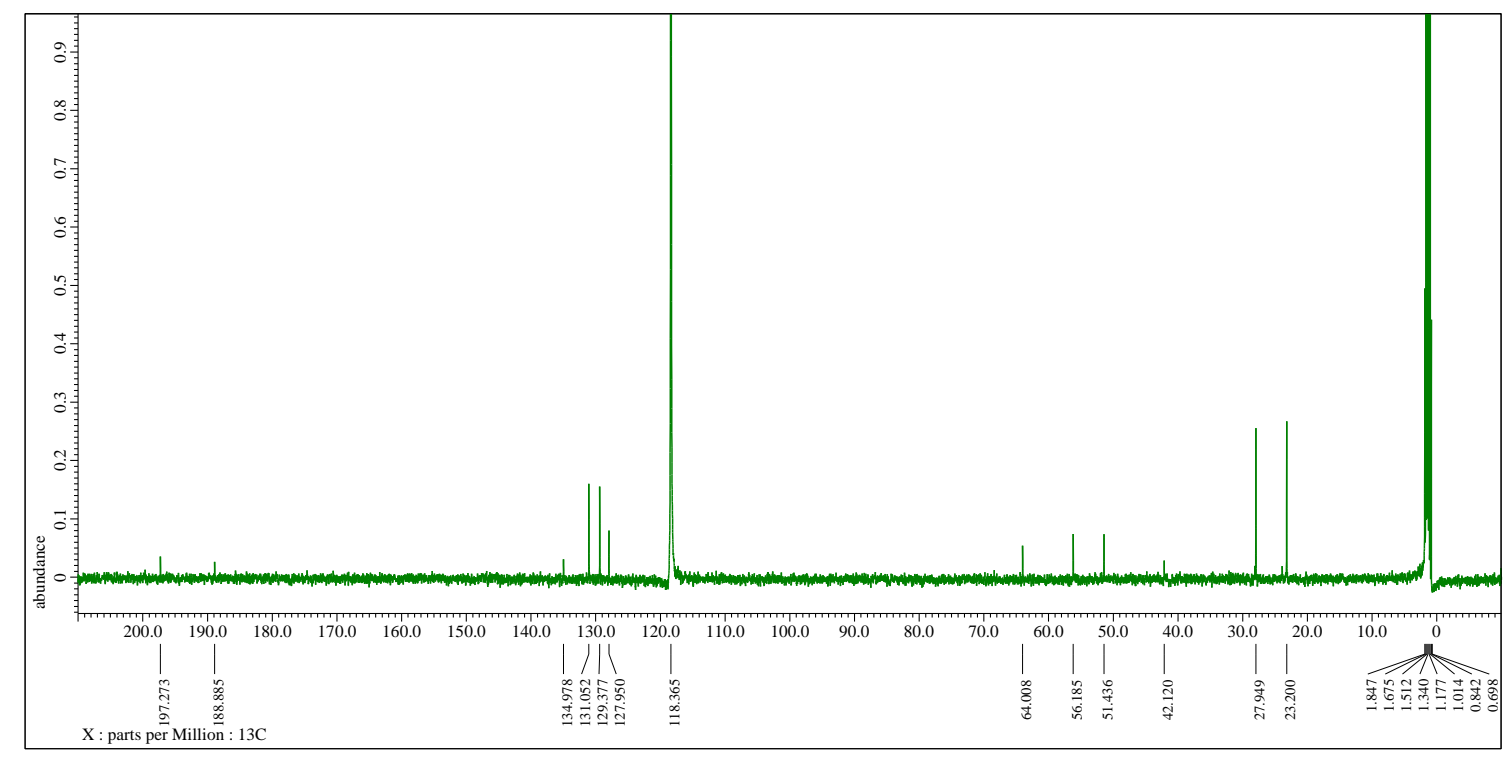


$(R, E)-N$-(tert-Butyl(oxo)(prop-1-en-1-yl)- $\lambda^{6}$-sulfanylidene)pivalamide (6j)

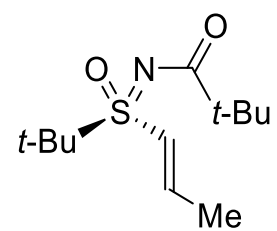

${ }^{1} \mathrm{H}$ NMR $\left(500 \mathrm{MHz}, \mathrm{CDCl}_{3}\right)$
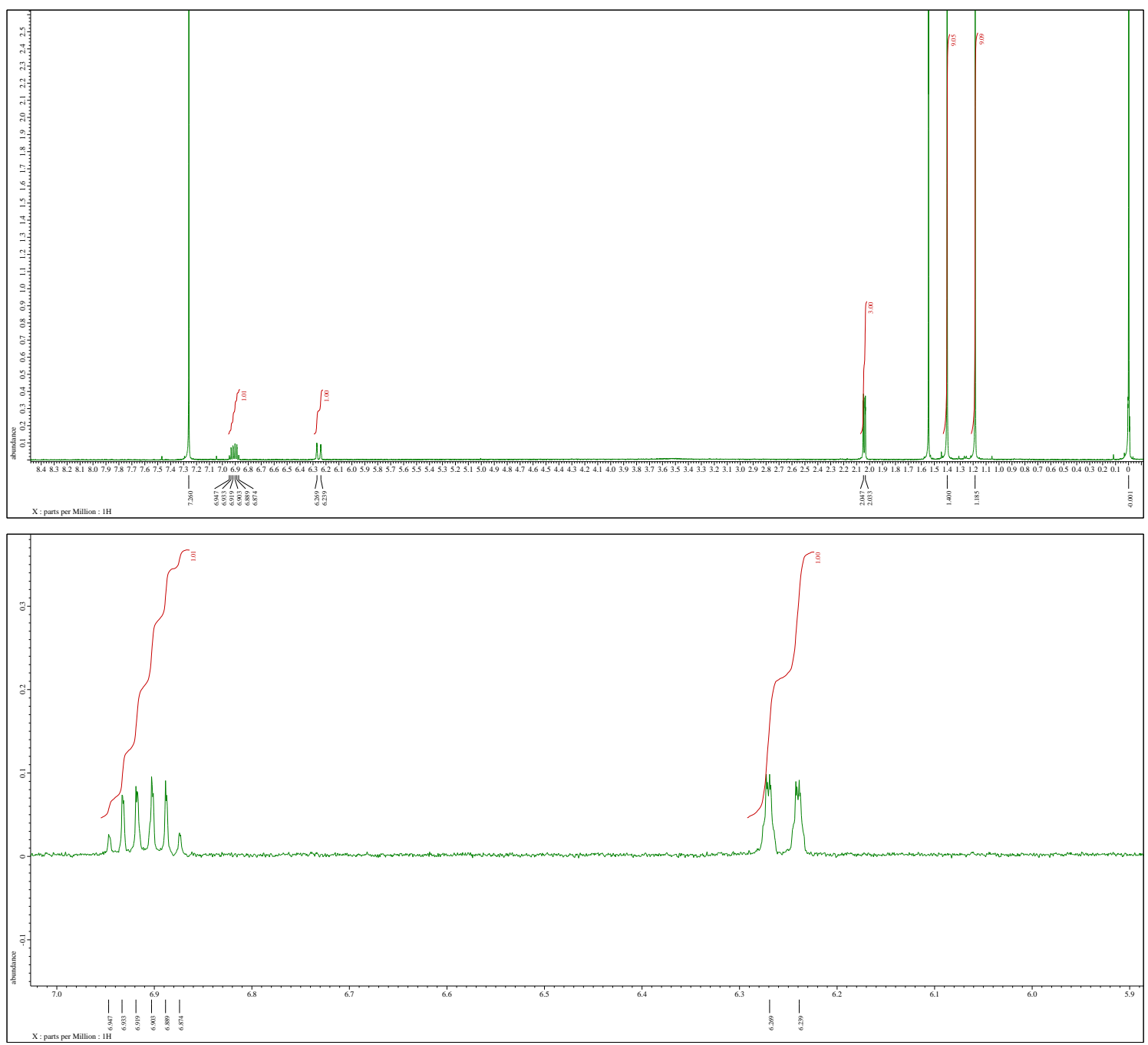


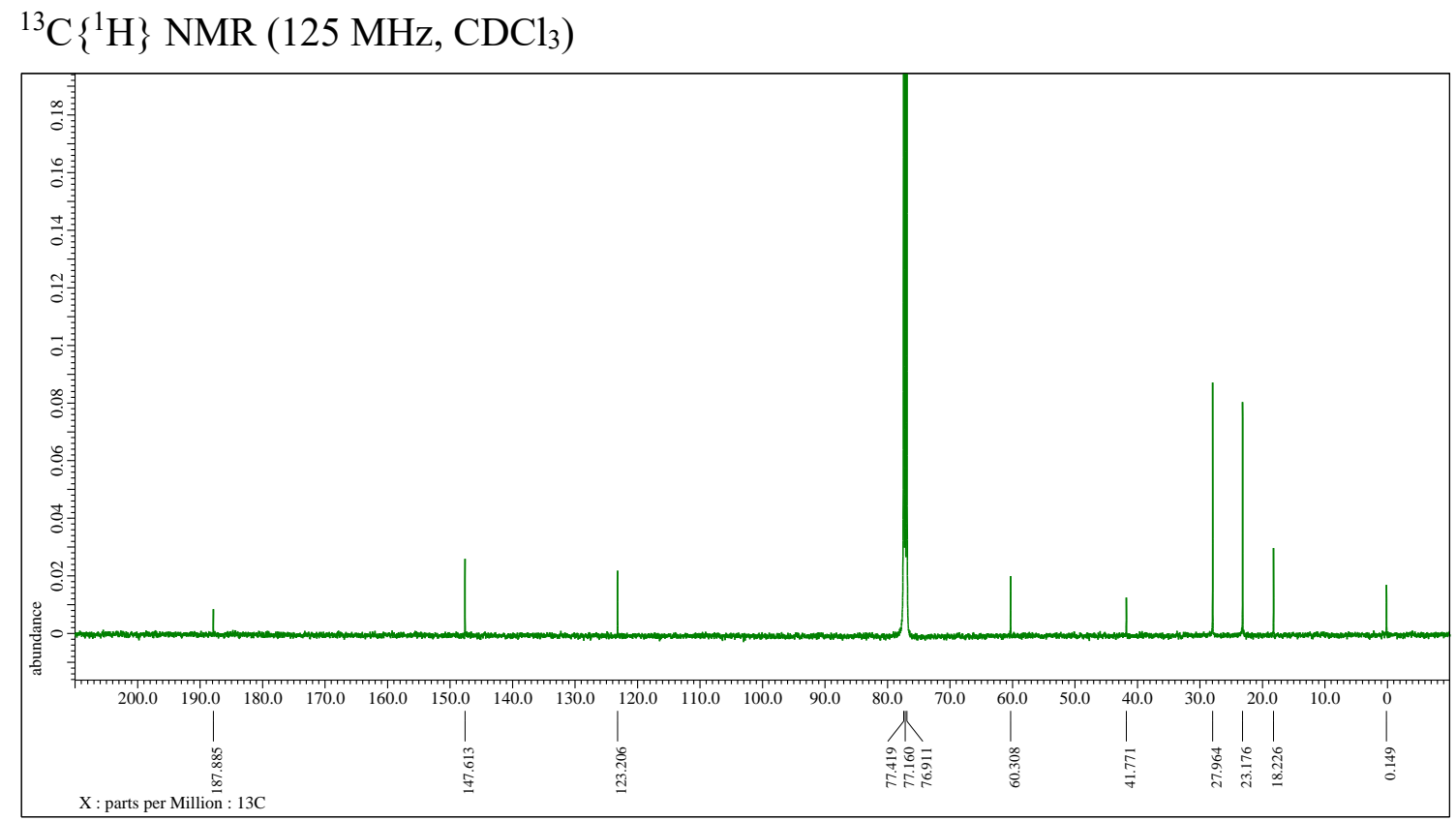


(R)-N-(tert-Butyl(oxo)(2-methylprop-1-en-1-yl)- $\lambda^{6}$-sulfanylidene)pivalamide (6m)

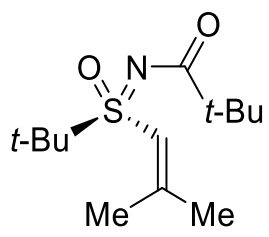

${ }^{1} \mathrm{H}$ NMR $\left(500 \mathrm{MHz}, \mathrm{CDCl}_{3}\right)$
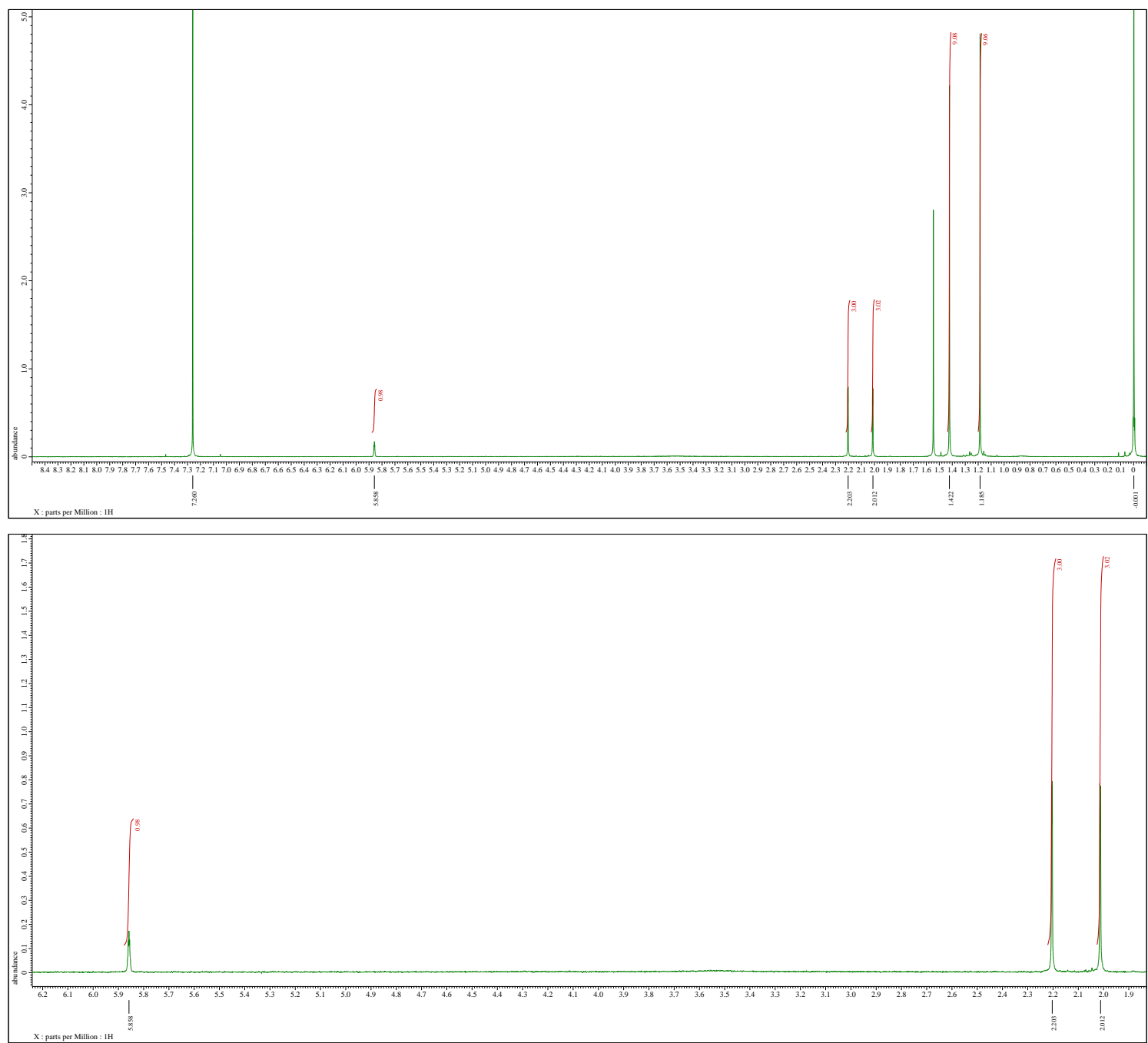
${ }^{13} \mathrm{C}\left\{{ }^{1} \mathrm{H}\right\}$ NMR $\left(125 \mathrm{MHz}, \mathrm{CDCl}_{3}\right)$

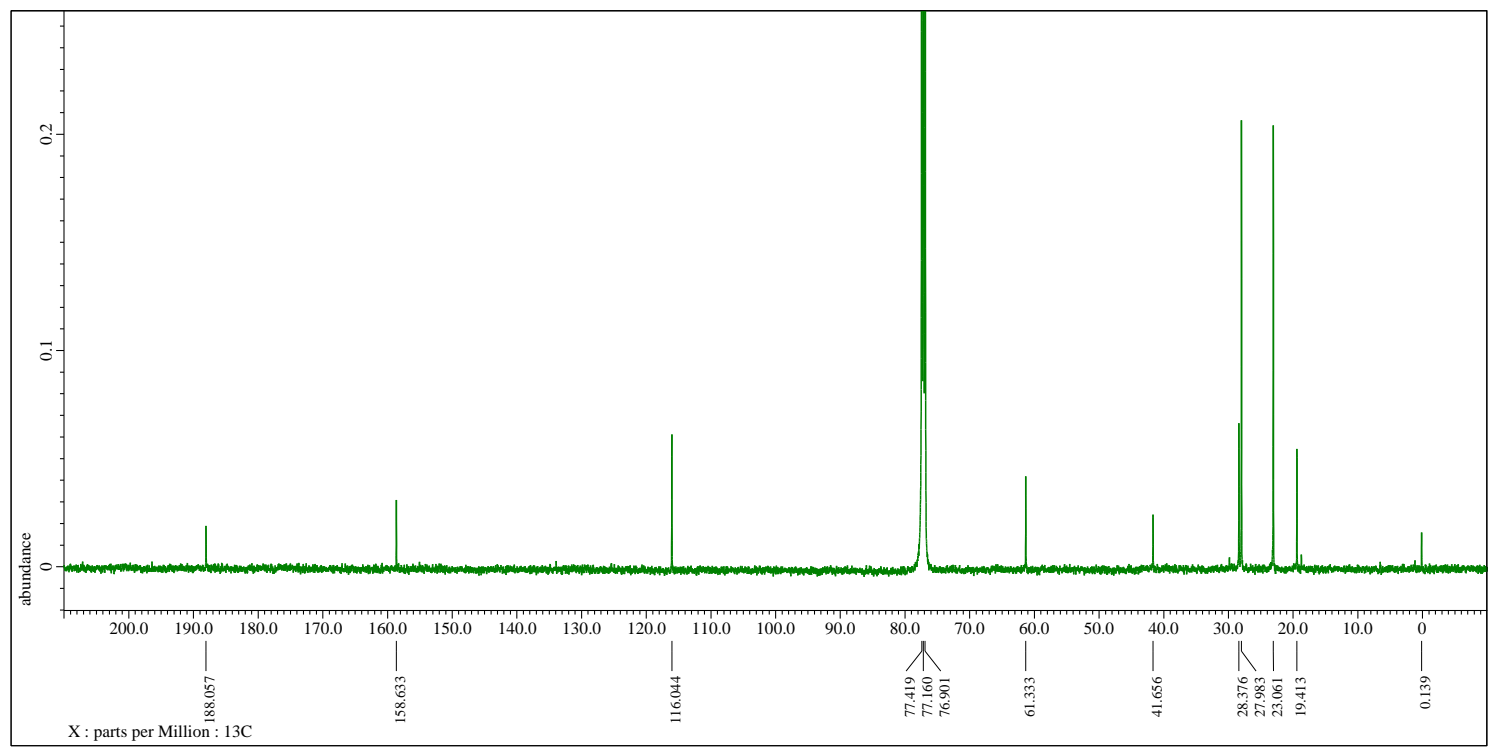




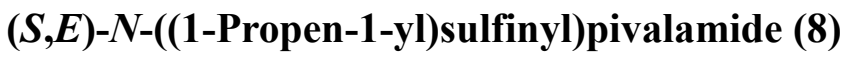<smiles>CC=CS(=O)NC(=O)C(C)(C)C</smiles>

${ }^{1} \mathrm{H} \mathrm{NMR}\left(500 \mathrm{MHz}, \mathrm{CDCl}_{3}\right)$

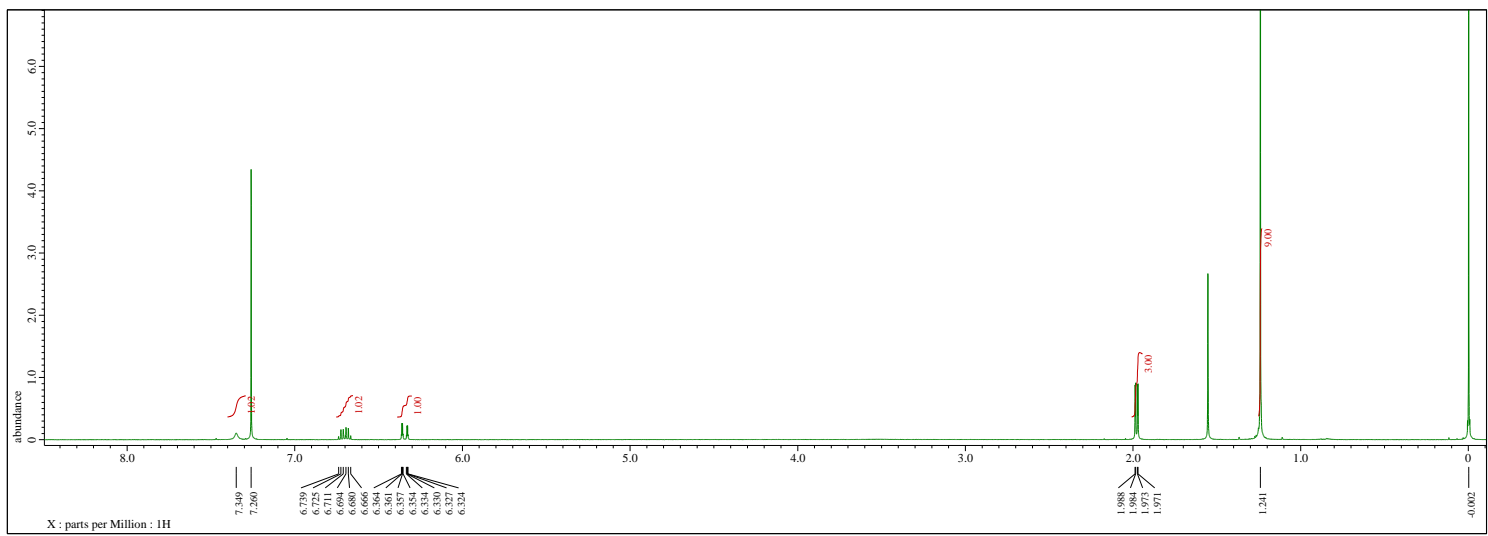

${ }^{13} \mathrm{C}\left\{{ }^{1} \mathrm{H}\right\} \mathrm{NMR}\left(125 \mathrm{MHz}, \mathrm{CDCl}_{3}\right)$

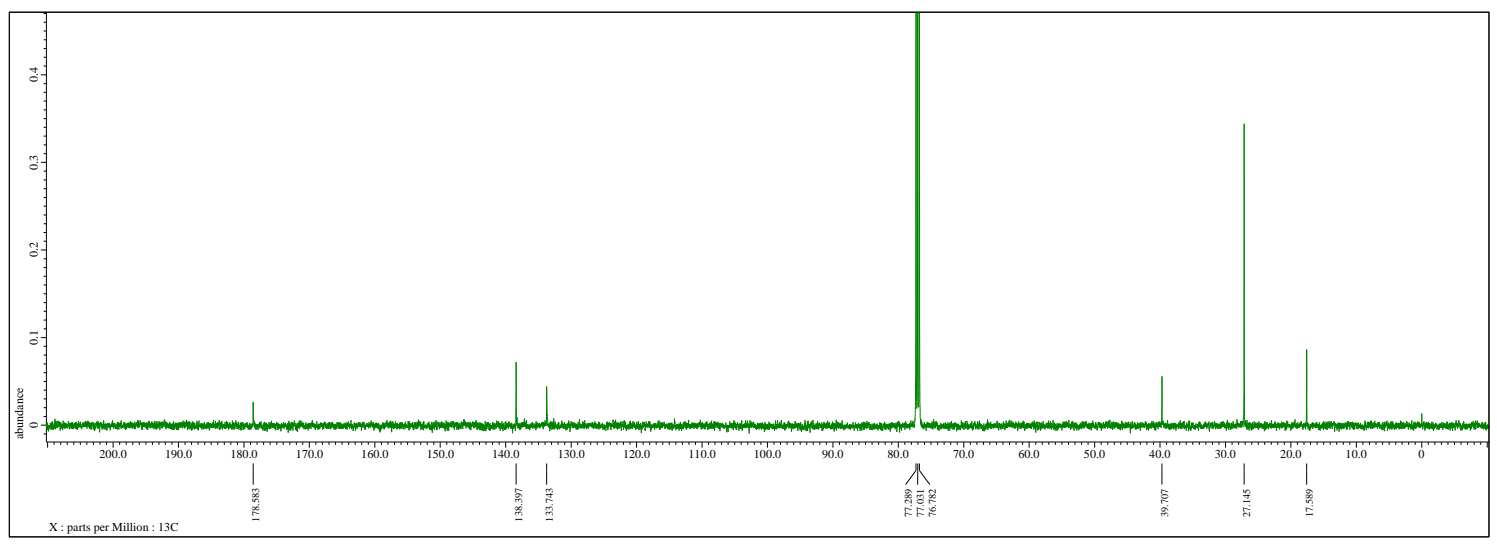


(S)-N-(Butyl(ethyl)(oxo)- $\lambda^{6}$-sulfanylidene)pivalamide (9)

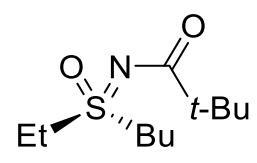

${ }^{1} \mathrm{H}$ NMR $\left(500 \mathrm{MHz}, \mathrm{CDCl}_{3}\right)$

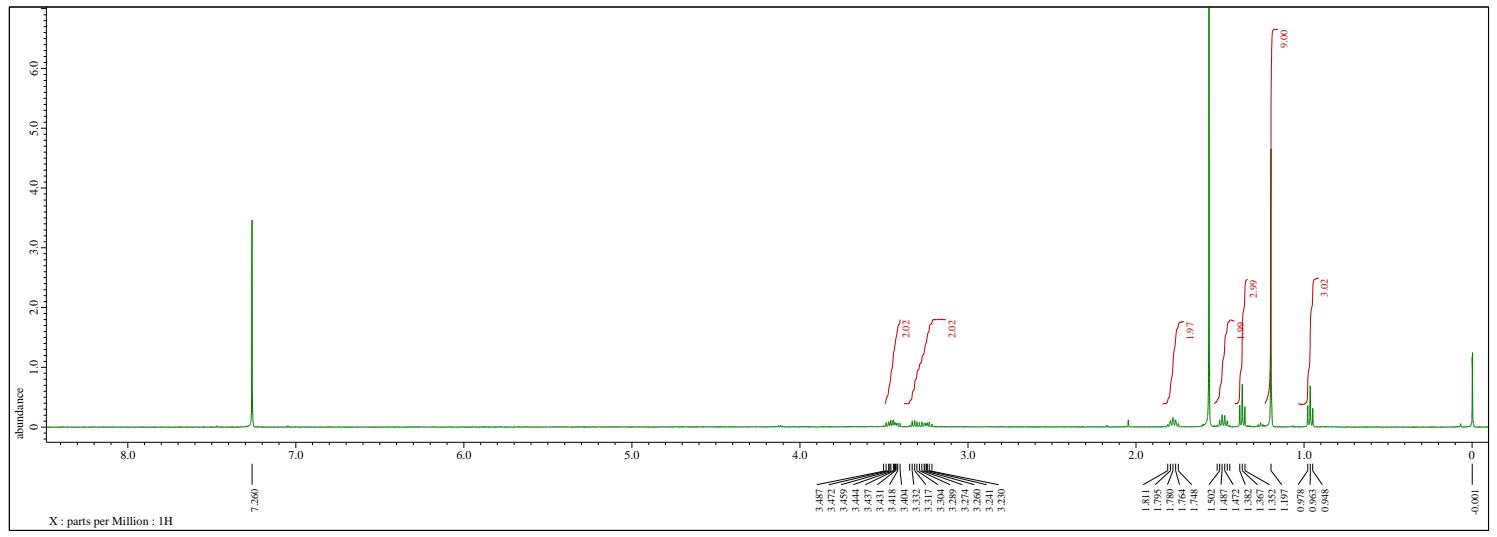


(S)-N-(p-tolylsufinyl)pivalamide (10)

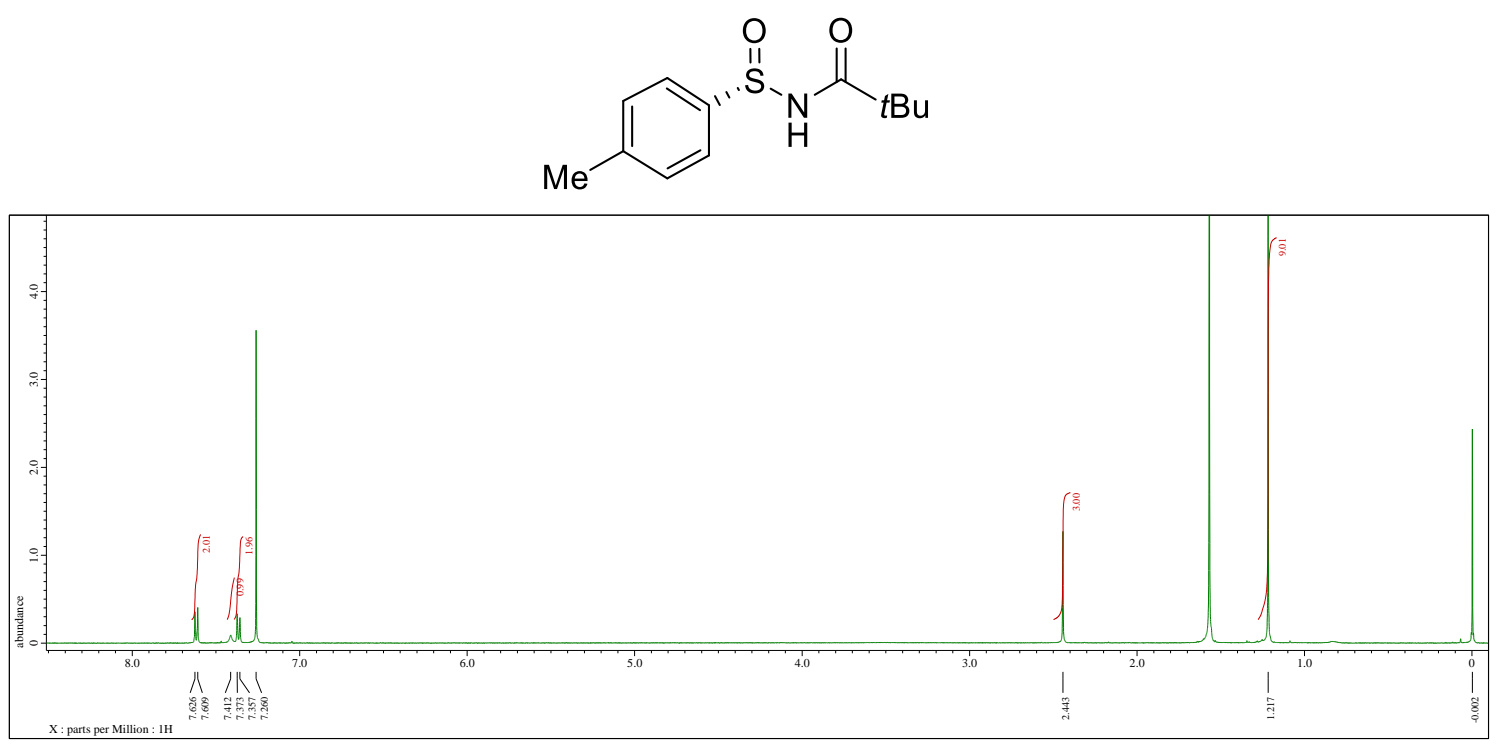


(S)-N-(Ethyl(oxo)(p-tolyl)- $\lambda^{6}$-sulfanylidene)pivalamide (11)

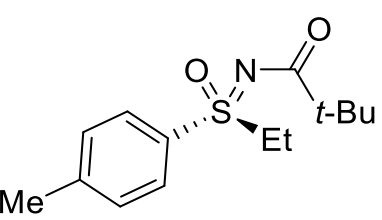

${ }^{1} \mathrm{H}$ NMR $\left(500 \mathrm{MHz}, \mathrm{CDCl}_{3}\right)$

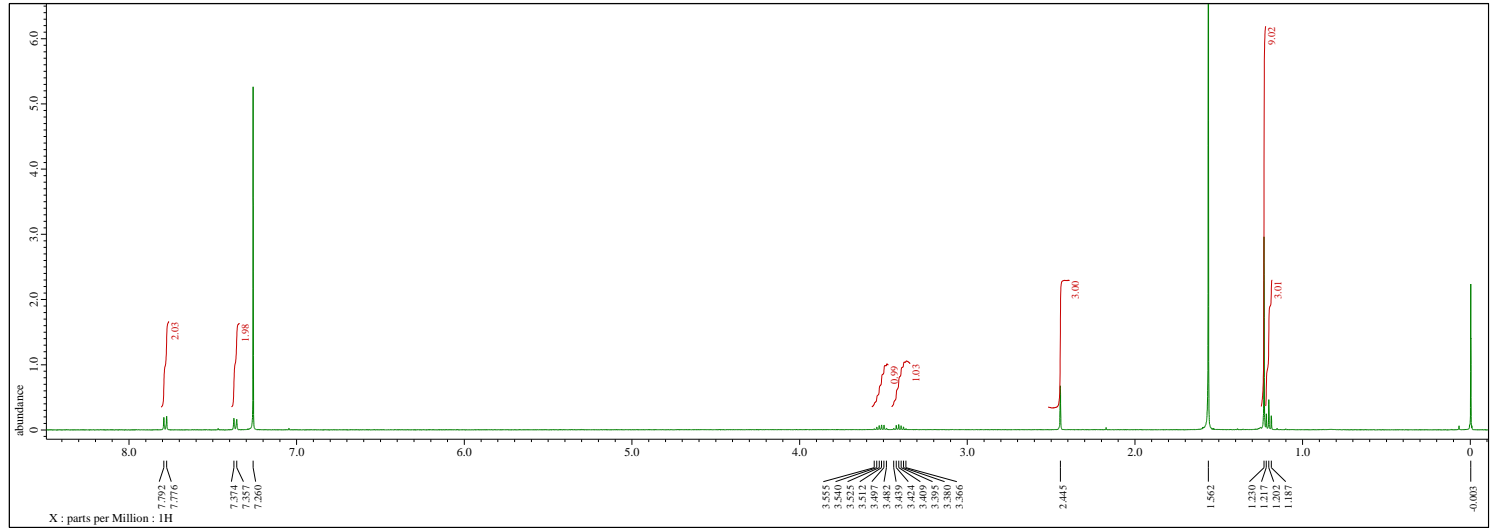


${ }^{1} \mathrm{H}-\mathrm{NMR}$ spectra of NMR experiment
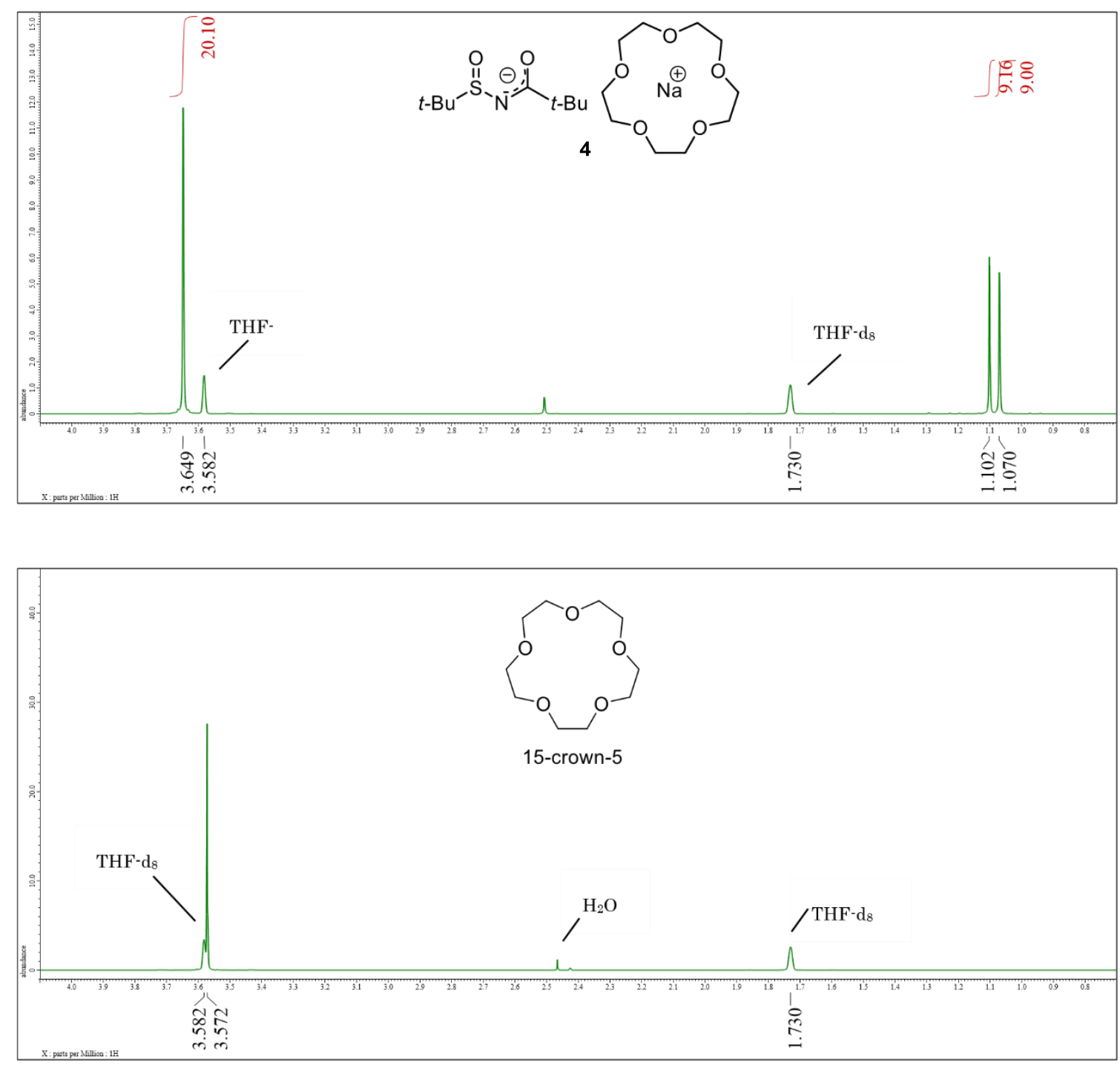

Figure S1. ${ }^{1} \mathrm{H}-\mathrm{NMR}$ spectra of $\mathbf{4}$ and 15 -crown-5 in THF-d $\mathrm{d}_{8}$ at room temperature. 


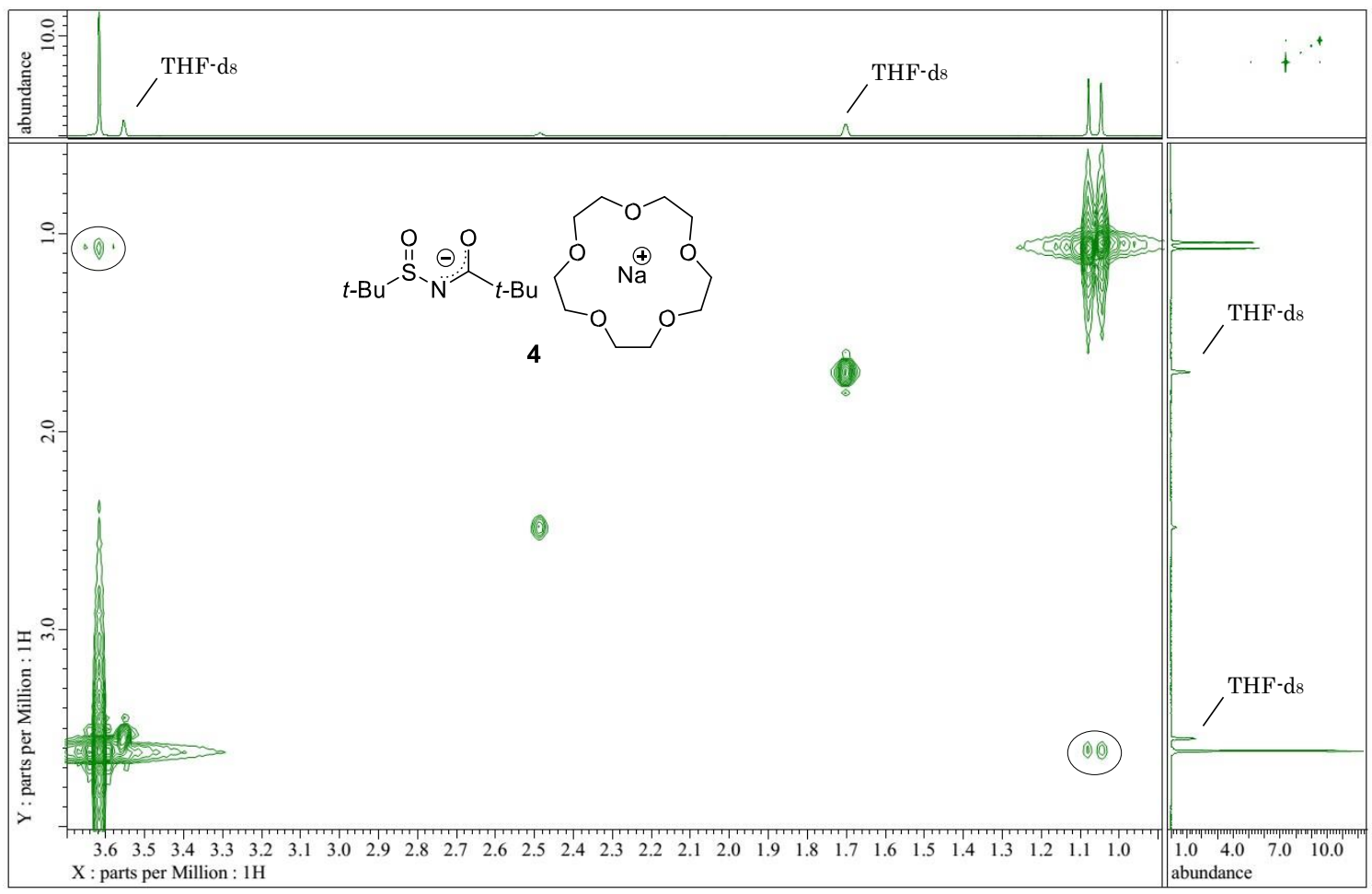

Figure S2. NOESY spectrum of $\mathbf{4}$ in THF-d 8 at room temperature.

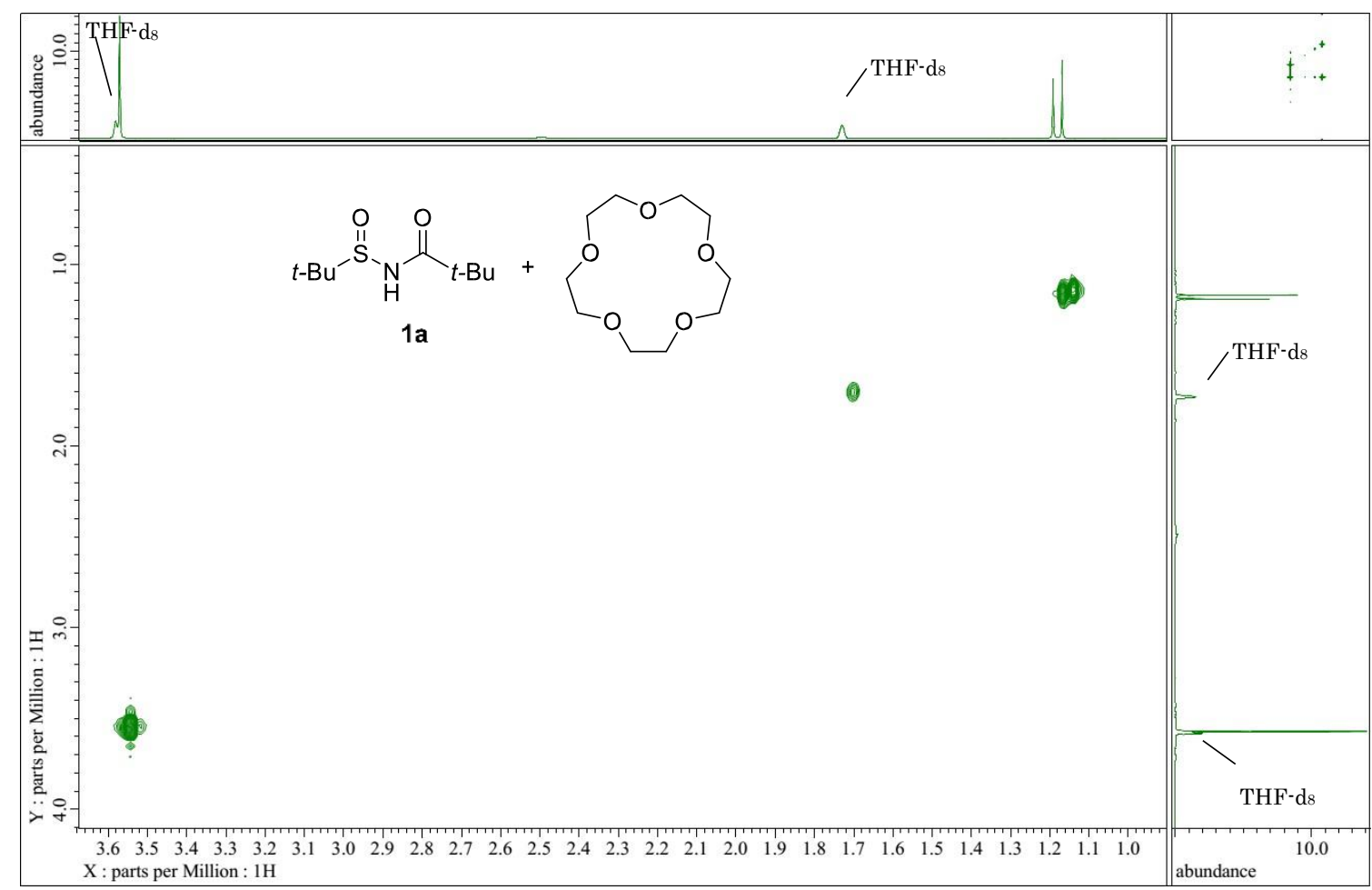

Figure S3. NOESY spectrum of $1: 1$ mixture of sulfinamide 1a and 15-crown-5 in THF$\mathrm{d}_{8}$ at room temperature. 
HPLC charts of mechanistic investigation for racemization

(rac)-N-(tert-Butylsulfinyl)pivalamide ((rac)-1a)<smiles>CC(C)(C)C(=O)NS(=O)C(C)(C)C</smiles>

(rac)-1a

HPLC analysis: Daicel CHIRALPAK IC-3, hexane $/ \mathrm{PrOH}=3 / 1$, flow rate $0.75 \mathrm{~mL} / \mathrm{min}$, $\lambda=208 \mathrm{~nm}$, retention time: $14.7 \mathrm{~min}$ (major) and $26.0 \mathrm{~min}$ (minor).

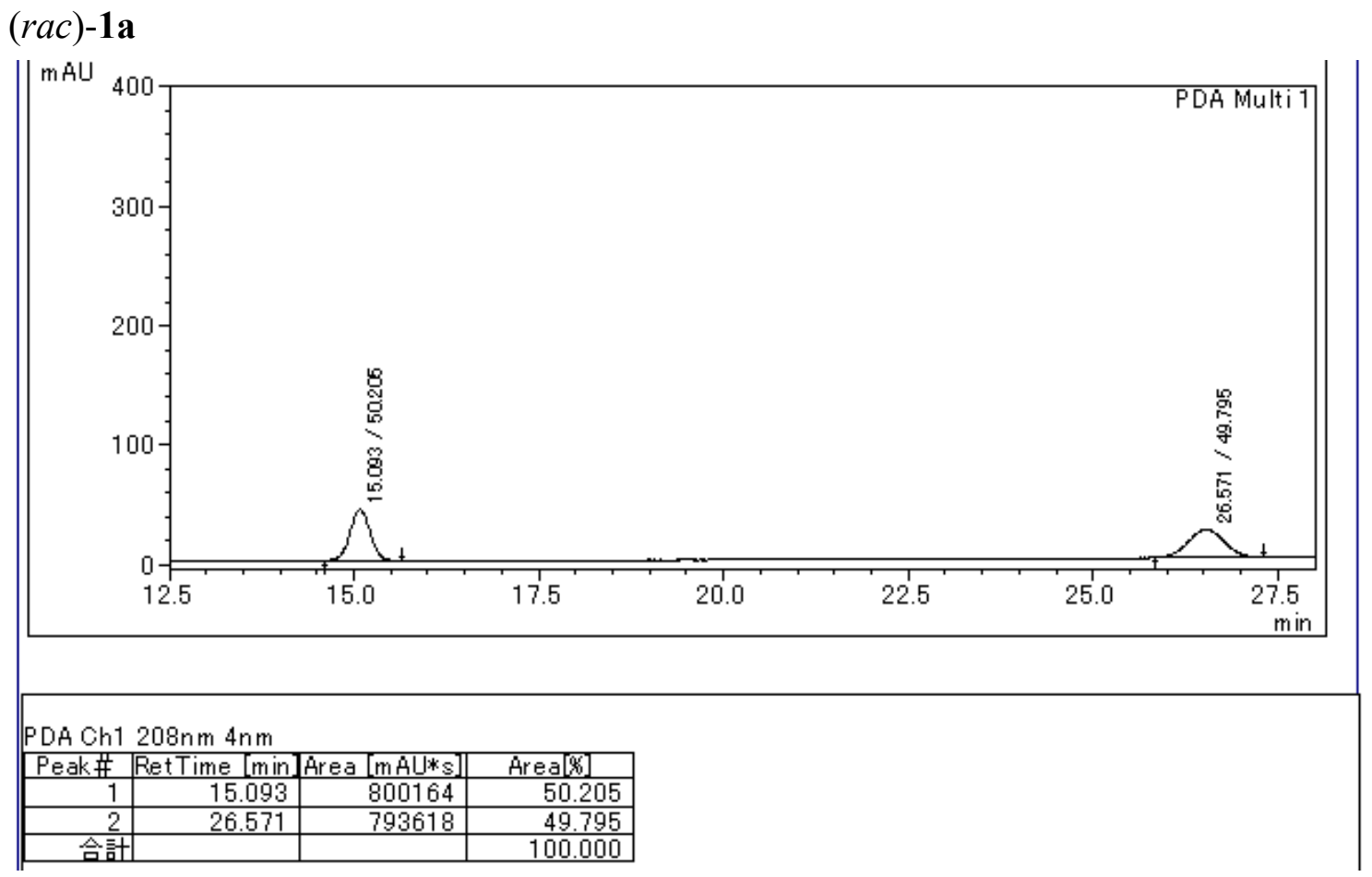


(R)-1a (After the reaction shown in Scheme 2)

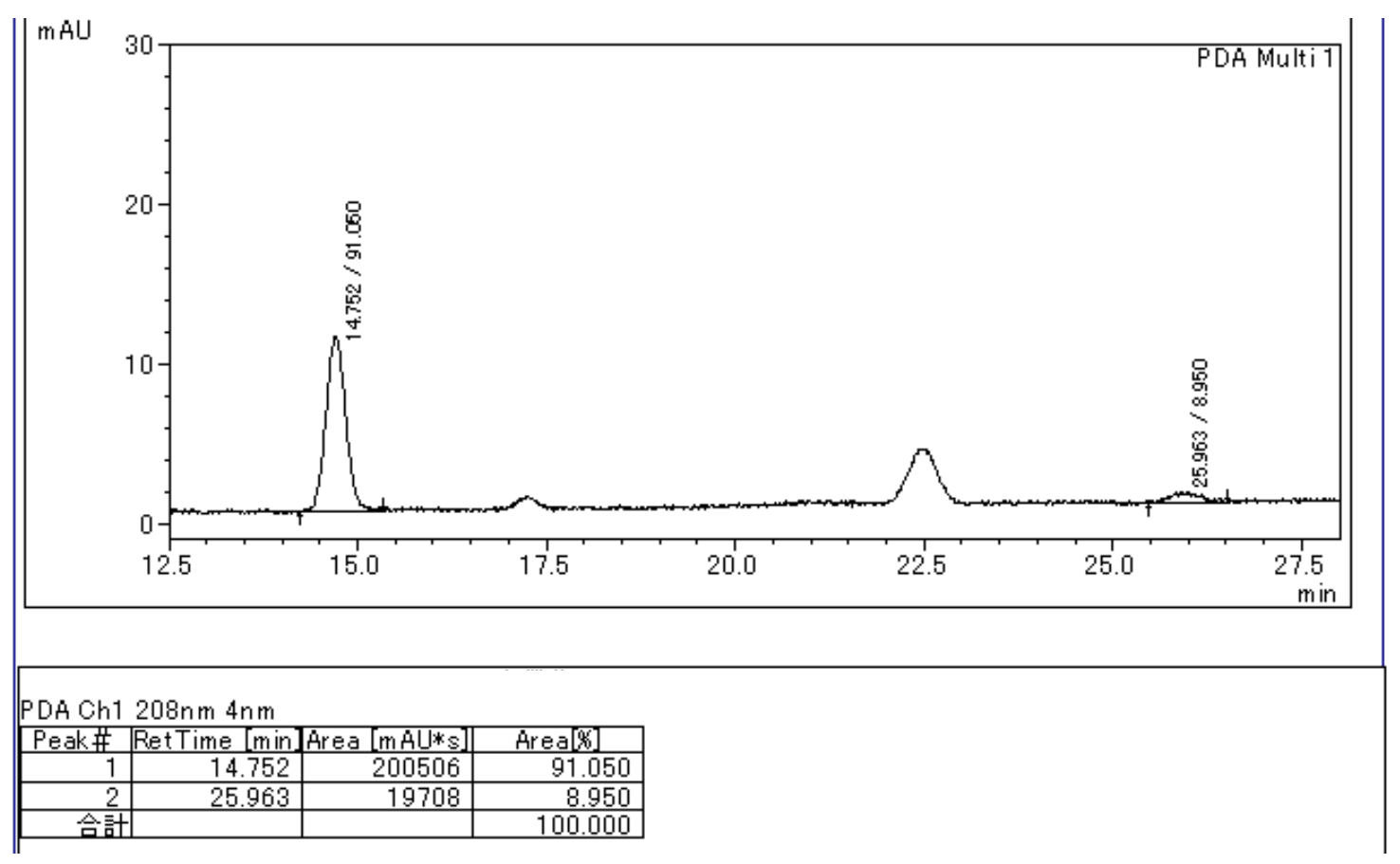


(rac)-N-(tert-Butyl(ethoxy)- $\lambda^{4}$-sulfanylidene)pivalamide ((rac)-5)

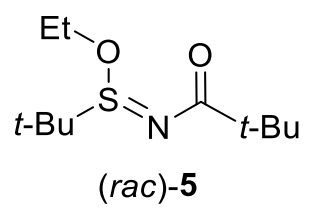

HPLC analysis: Daicel CHIRALPAK IG, hexane/2-propanol $=10 / 1$, flow rate 0.75 $\mathrm{mL} / \mathrm{min}, \lambda=254 \mathrm{~nm}$, retention time: $5.8 \mathrm{~min}$ (minor) and $6.3 \mathrm{~min}$ (major).

(rac)-5

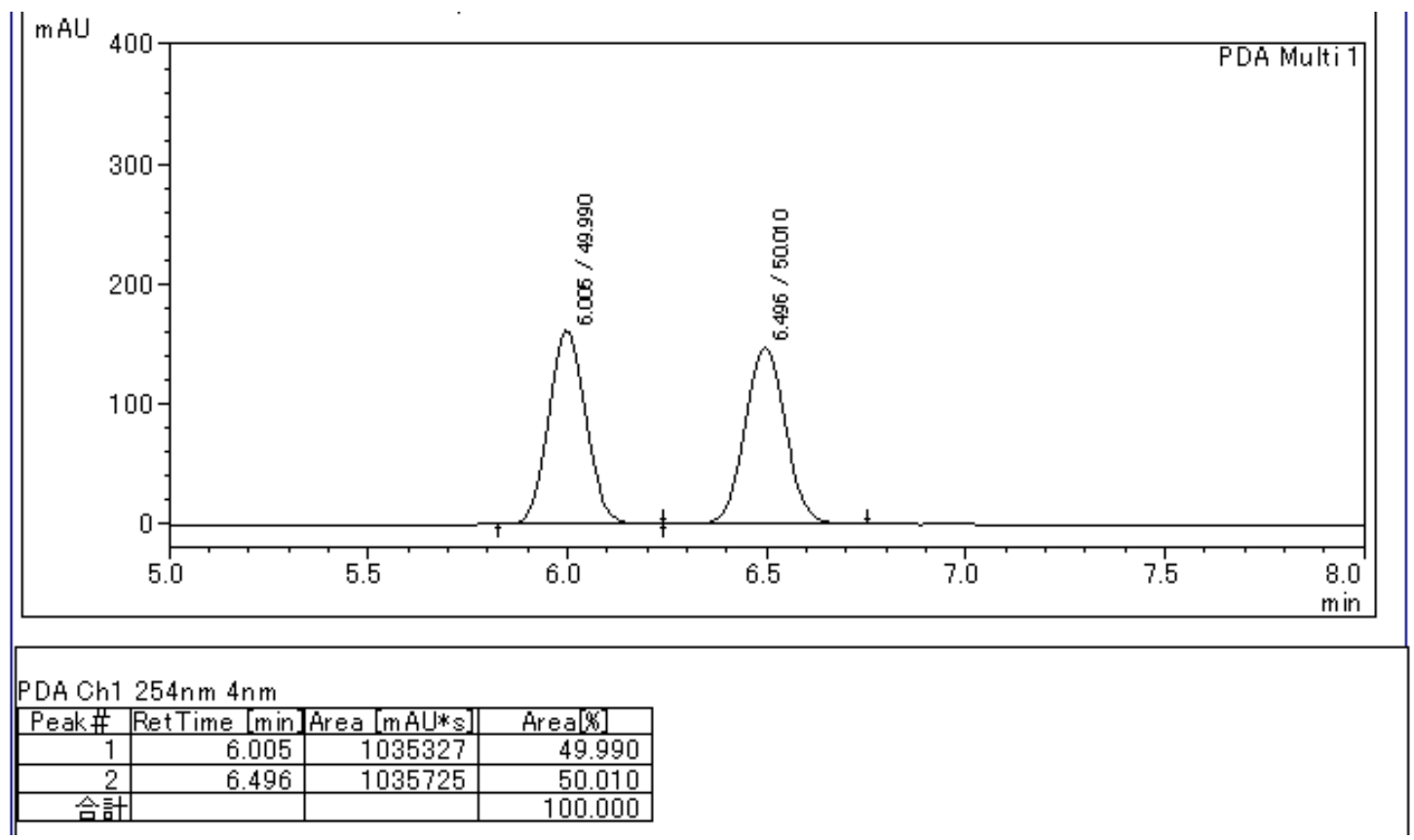


(S)-5 (Before the reaction shown in Scheme 2)

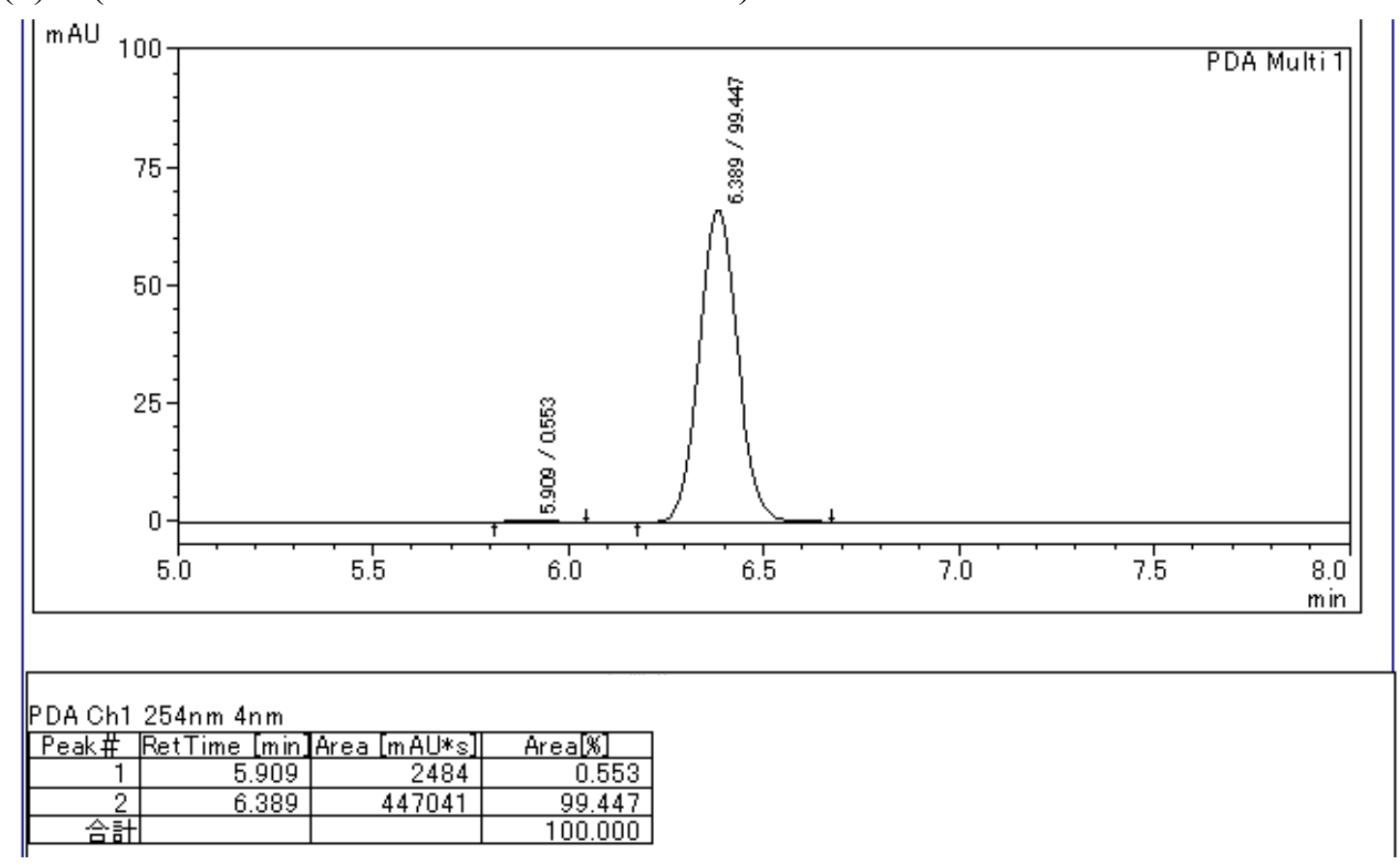

(S)-5 (After the reaction shown in Scheme 2)

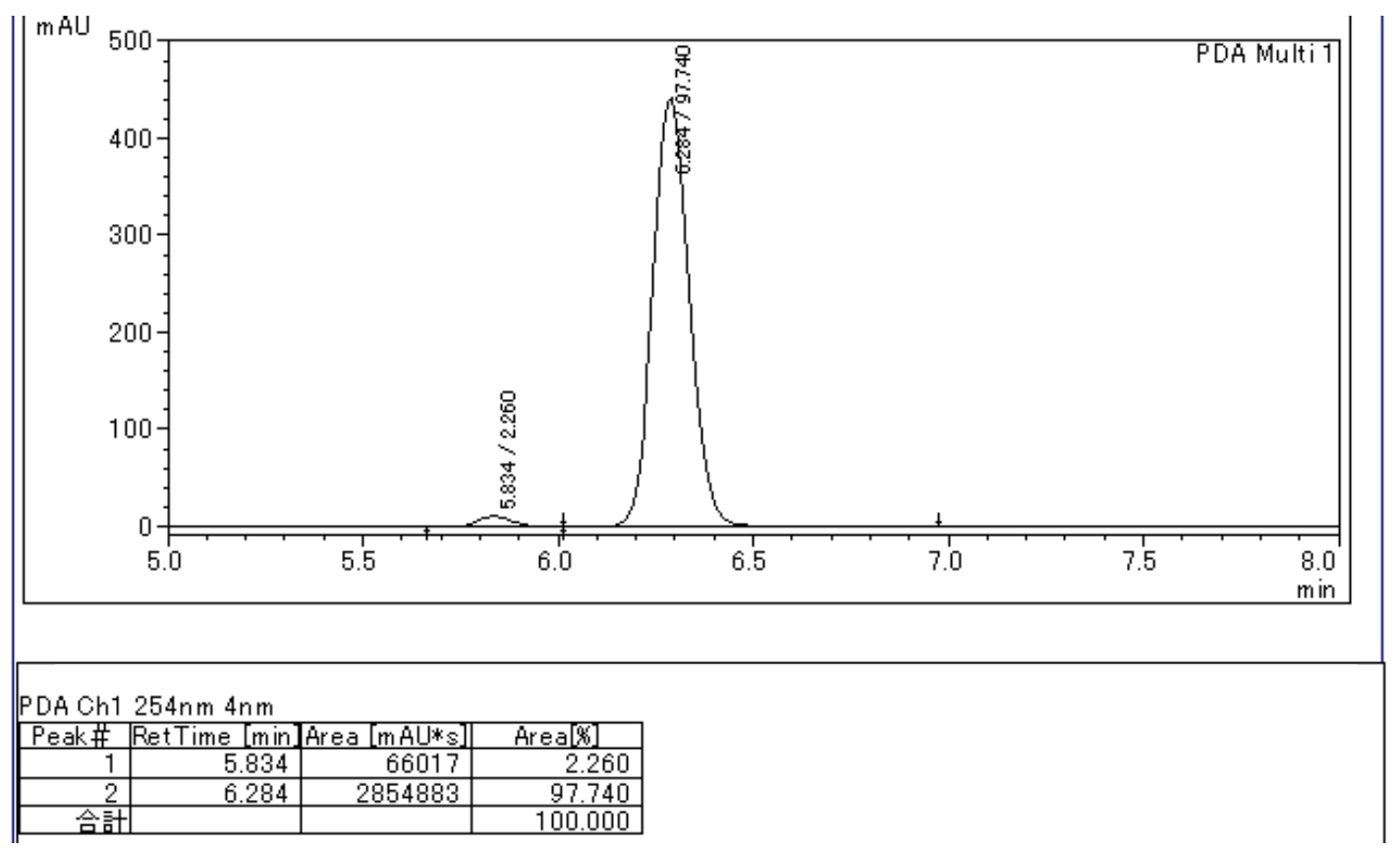


<smiles>CC(C)(C)C(=O)NS(=O)C(C)(C)C</smiles>

$(S)-1 \mathbf{a}(>99 \%$ ee $)$

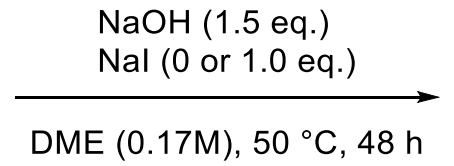

$t-\mathrm{Bu}^{\prime \prime} \mathrm{S}_{\mathrm{H}}^{\mathrm{O}} \mathrm{N}_{t-\mathrm{Bu}}^{\mathrm{O}}$

$(S)-1 \mathbf{a}(>99 \%$ ee $)$

(rac)-N-(tert-Butylsulfinyl)pivalamide ((rac)-1a)<smiles>CC(C)(C)C(=O)NS(=O)C(C)(C)C</smiles>

$($ rac)-1a

HPLC analysis: Daicel CHIRALPAK IC, hexane $/ \mathrm{PrOH}=1 / 1$, flow rate $0.75 \mathrm{~mL} / \mathrm{min}, \lambda$ $=208 \mathrm{~nm}$, retention time: $8.2 \mathrm{~min}$ and $12.0 \mathrm{~min}$.

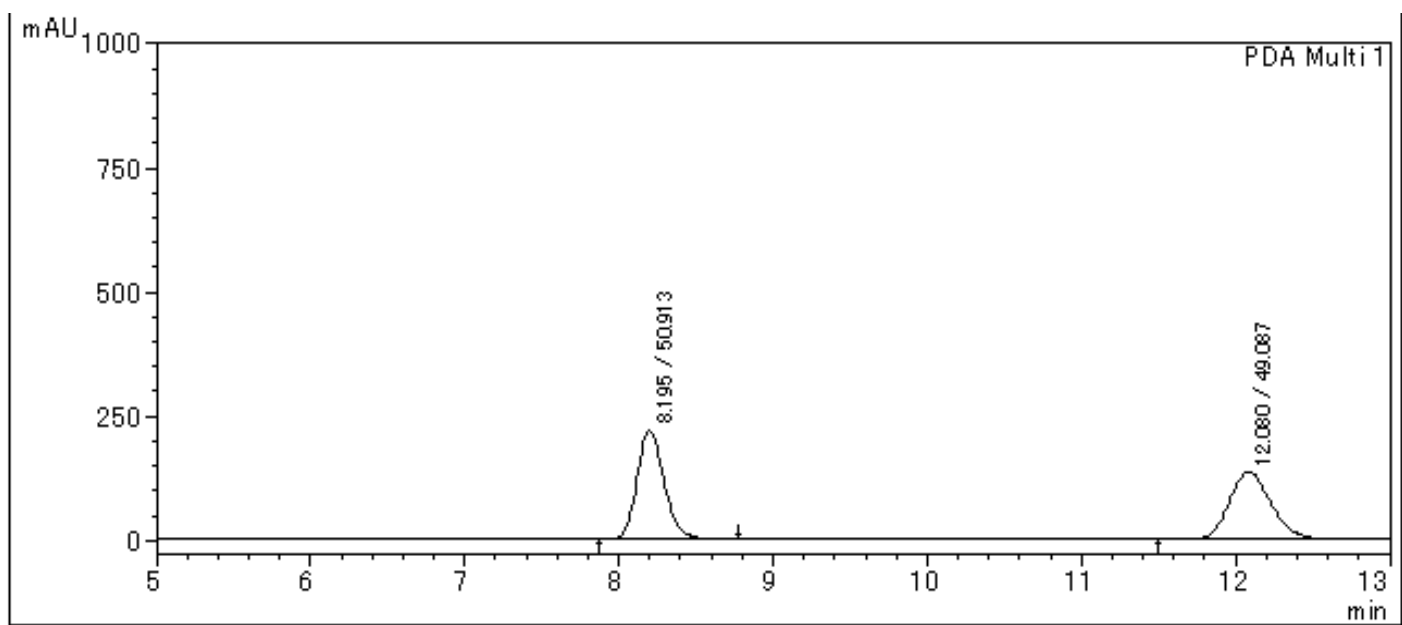

〈ビーグボート〉

\begin{tabular}{|c|c|c|c|c|}
\hline & & & & \\
\hline E゚ーל\# & 保持時間 & 面皘 & 面䞍夥 & \\
\hline 1 & 8.195 & 2659678 & 50.913 & \\
\hline 2 & 12.080 & 2564239 & 49.087 & \\
\hline 合計 & & 5223917 & 100.000 & \\
\hline
\end{tabular}


(S)-1a (After the reaction without $\mathrm{NaI}$ )

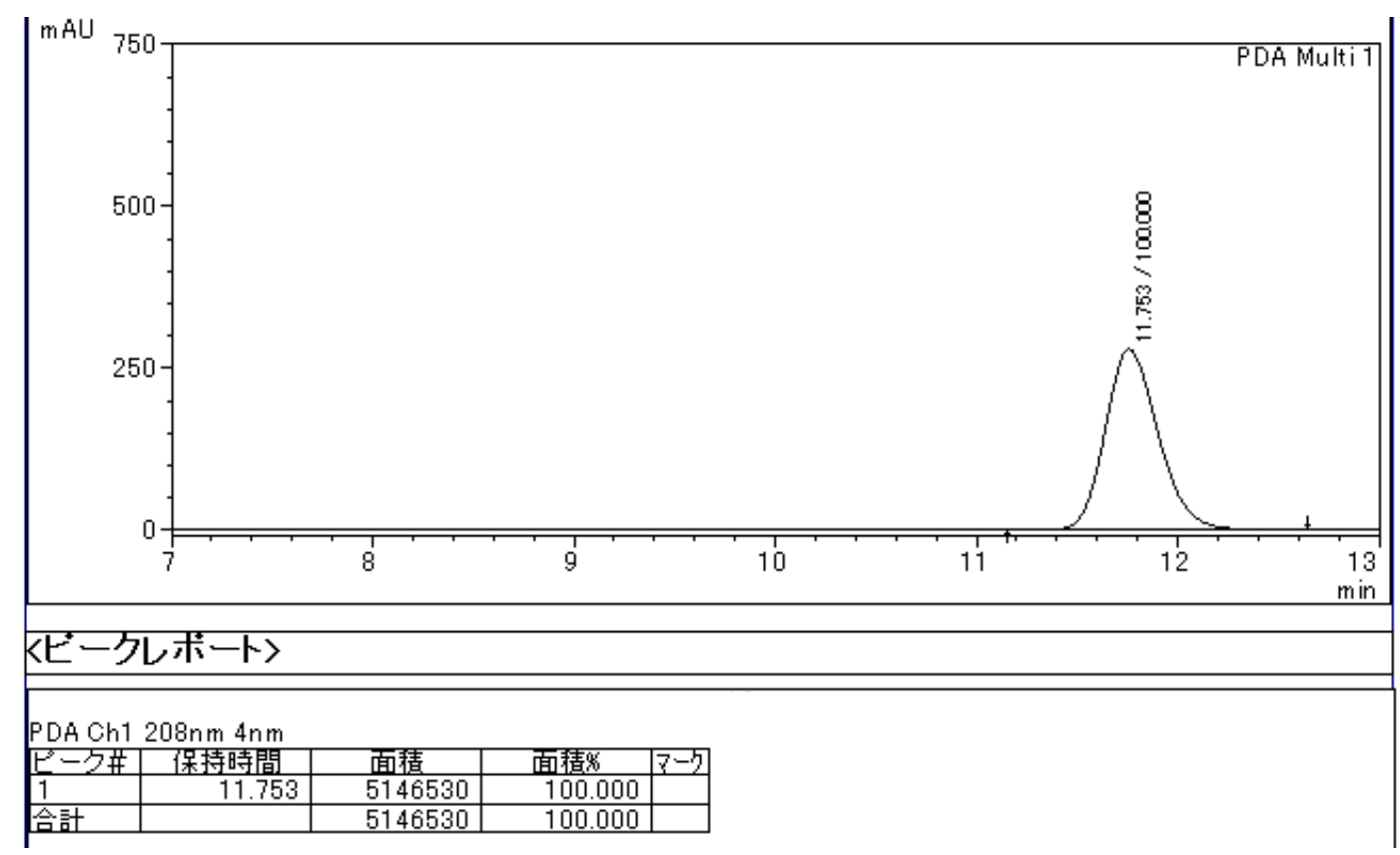

(S)-1a (After the reaction with $\mathrm{NaI}$ )

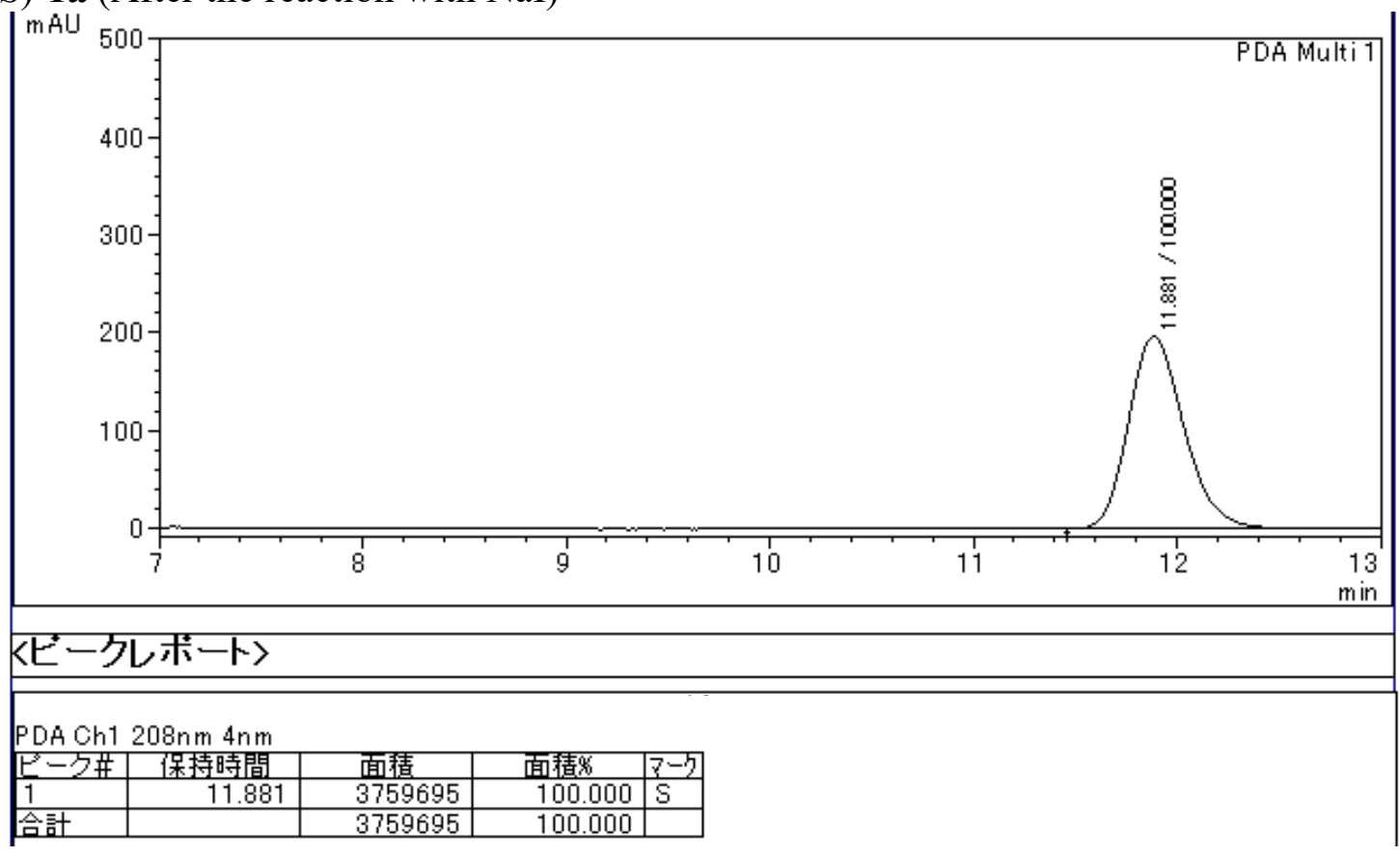


<smiles>CCS(=O)(=O)NC(=O)C(C)(C)C</smiles>

$(S)-2 \mathbf{a}(>99 \%$ ee $)$

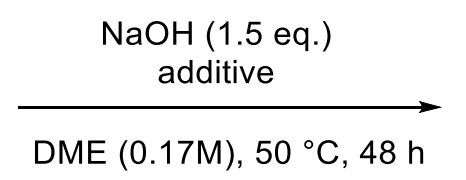

without additive

Et-I (5.0 eq.)

Nal (1.0 eq.)

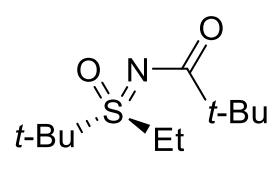

$(S)-2 \mathbf{a}(>99 \%$ ee $)$

$(>99 \%$ ee $)$

$(>99 \%$ ee $)$

(rac)- $N$-(tert-Butyl(ethyl)(oxo)- $\lambda^{6}$-sulfanylidene)pivalamide ((rac)-2a)<smiles>CC[R15](C)(C)C(=O)N=S(=O)(CC)C(C)(C)C</smiles>

HPLC analysis: Daicel CHIRALPAK IC, hexane $/ \mathrm{PrOH}=1 / 1$, flow rate $0.75 \mathrm{~mL} / \mathrm{min}, \lambda$ $=210 \mathrm{~nm}$, retention time: $8.2 \mathrm{~min}$ (minor) and $9.7 \mathrm{~min}$ (major).

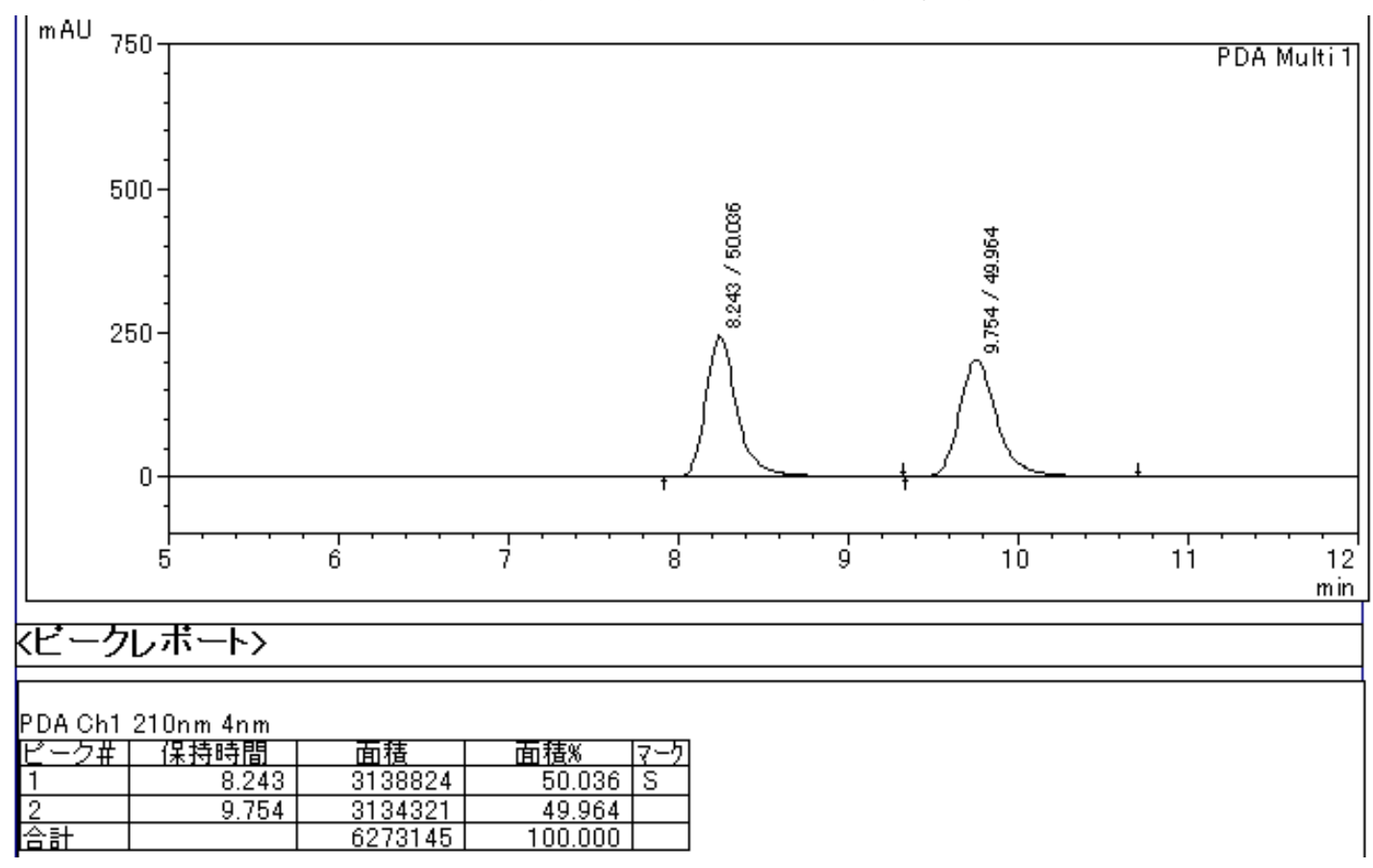


(S)-2a (After the reaction without additive)

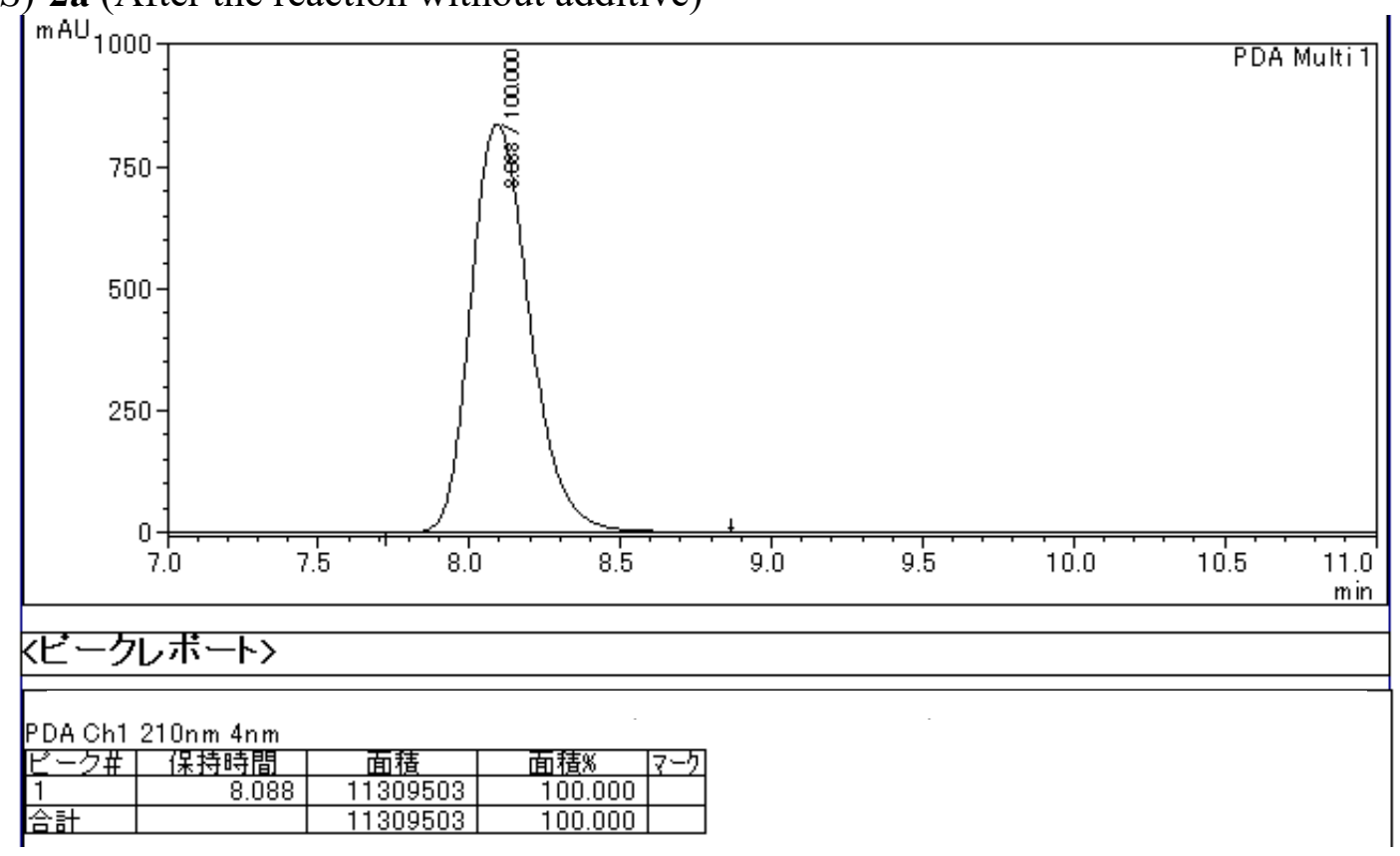

(S)-2a (After the reaction with Et-I)

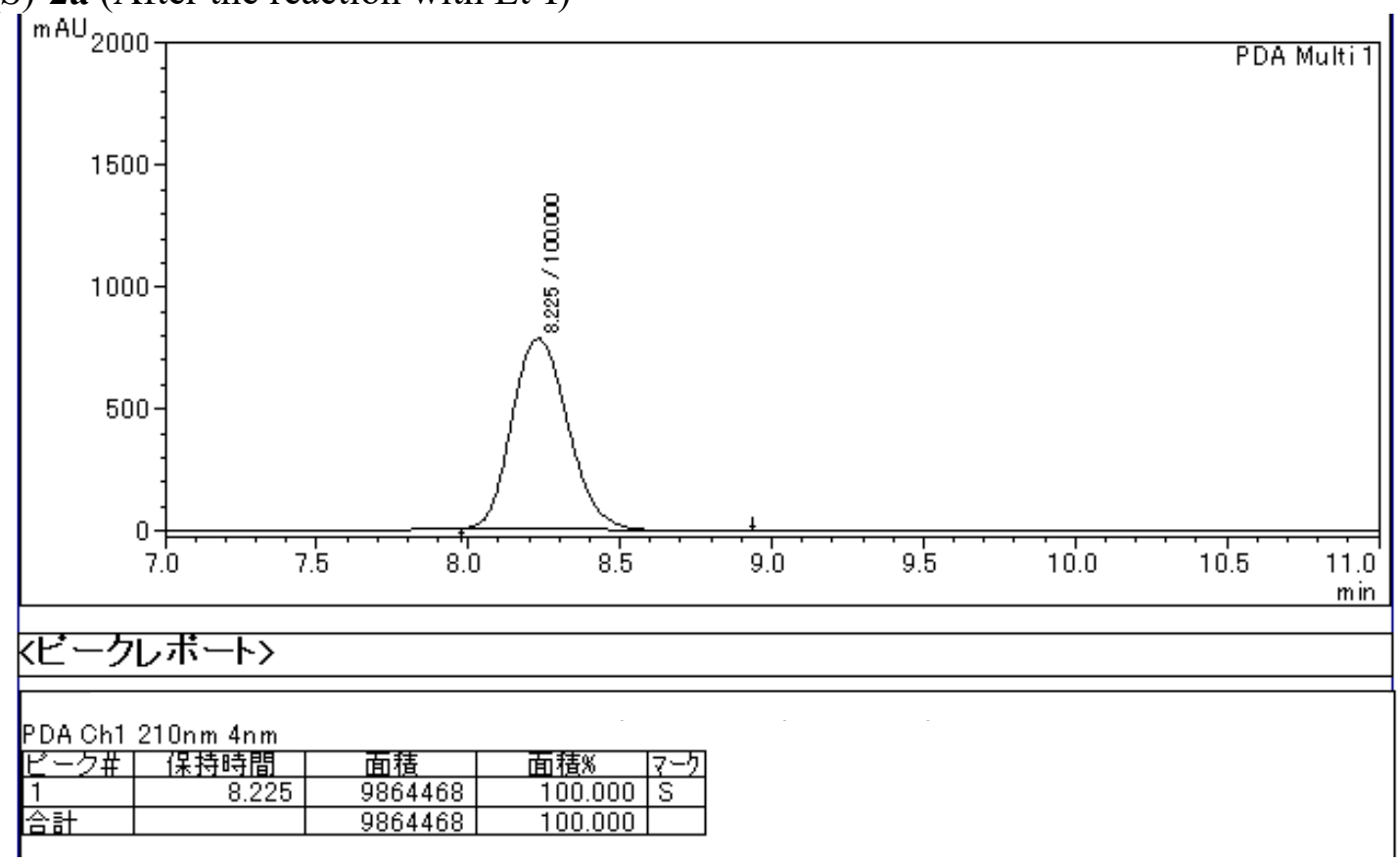


(S)-2a (After the reaction with $\mathrm{NaI}$ )

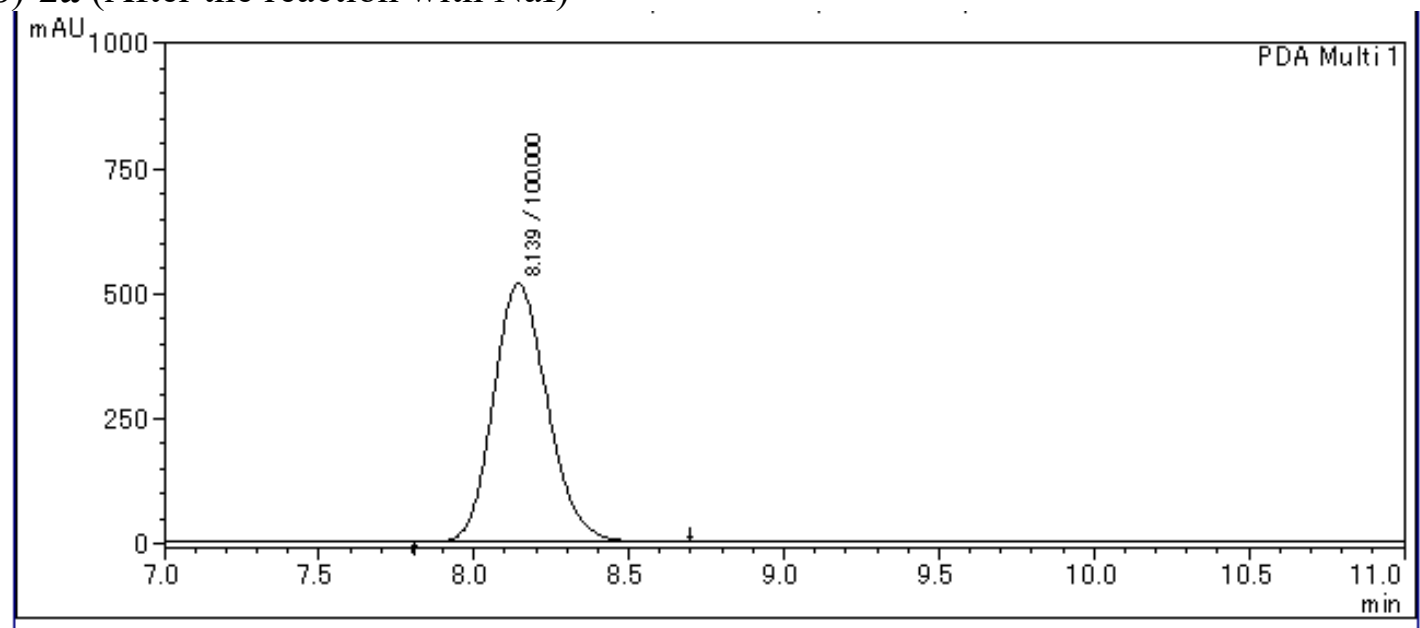

kピーグボート〉

PDA Ch1 $210 \mathrm{~nm} 4 \mathrm{~nm}$

\begin{tabular}{|c|c|c|c|c|}
\hline$E^{2}-2 \#$ & 保持時間 & 面皘 & 面啨夥 & $7-3$ \\
\hline 1 & 8.139 & 6242100 & 100.000 & \\
\hline 合計 & & 6242100 & 100.000 & \\
\hline
\end{tabular}




\section{HPLC charts of compounds}

(R)-N-(tert-Butylsulfinyl)pivalamide (1a)<smiles>CC(C)(C)C(=O)NS(=O)C(C)(C)C</smiles>

HPLC analysis: Daicel CHIRALPAK IC, hexane $/ \mathrm{PrOH}=1 / 1$, flow rate $0.75 \mathrm{~mL} / \mathrm{min}, \lambda$ $=208 \mathrm{~nm}$, retention time: $8.2 \mathrm{~min}$ (major) and $12.0 \mathrm{~min}$ (minor).

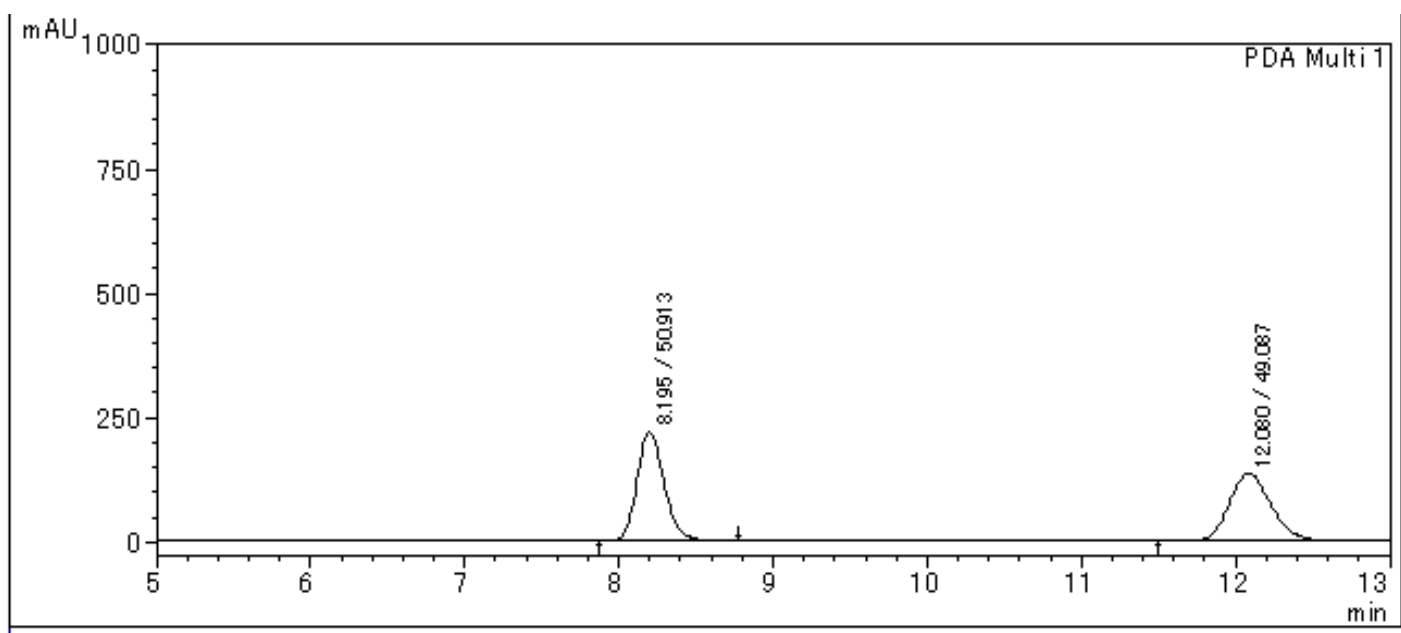

\section{〈ビークしボート〉}

PDÁ Ch1 208nm 4nm

\begin{tabular}{|c|c|c|c|c|}
\hline Éーク\# & 保持時間 & 面埥 & 面猜独 & $7-5$ \\
\hline 1 & 8.195 & 2659678 & 50.913 & \\
\hline 2 & 12.080 & 2564239 & 49.087 & \\
\hline 合計 & & 5223917 & 100.000 & \\
\hline
\end{tabular}

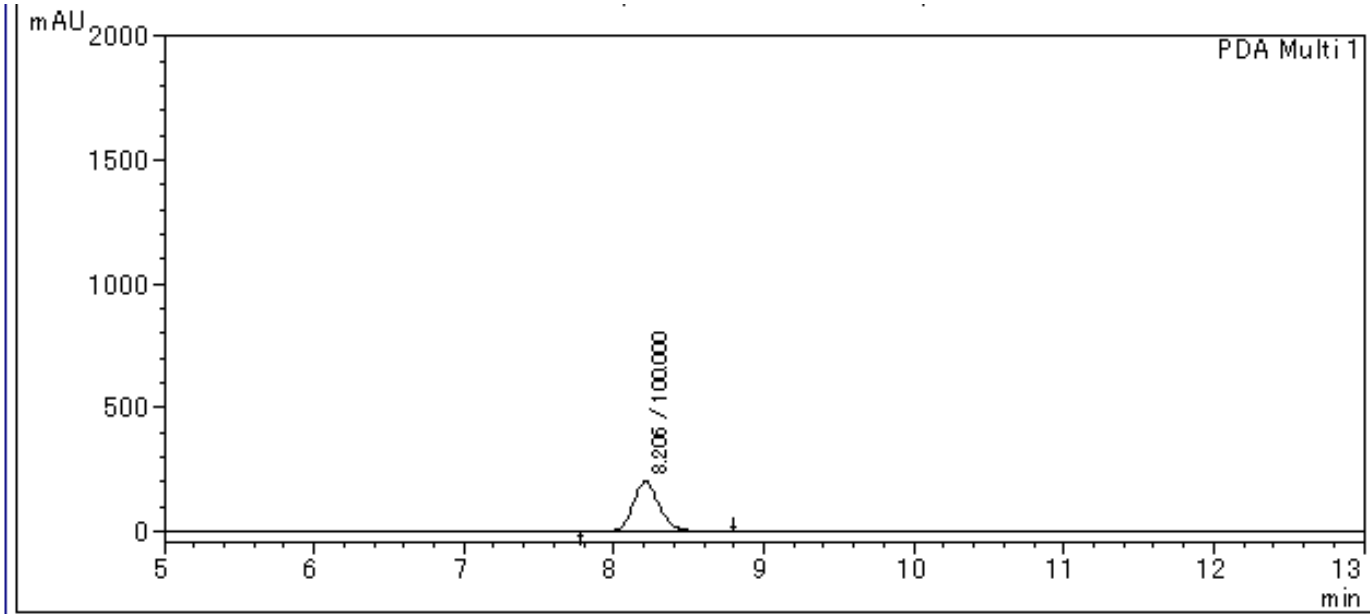

PDA Ch1 208nm 4nm

Peak\# RetTime [min]Area [mAU*s] Area $[\mathrm{X}]$

\begin{tabular}{|r|r|r|r|}
\hline Peak\# & RetTime [m in & Area [mAU*s] & AreaD, \\
\hline 1 & 8.206 & 2388929 & 100.000 \\
\hline 合計 & & & 100.000 \\
\hline
\end{tabular}


(R)- $N$-(tert-Butyl(ethyl)(oxo)- $\lambda^{6}$-sulfanylidene)pivalamide (2a)<smiles>CC[Sb](=O)(CC(C)(C)C)NC(=O)C(C)(C)C</smiles>

HPLC analysis: Daicel CHIRALPAK IC, hexane $/ \mathrm{PrOH}=1 / 1$, flow rate $0.75 \mathrm{~mL} / \mathrm{min}, \lambda$ $=210 \mathrm{~nm}$, retention time: $8.2 \mathrm{~min}$ (minor) and $9.7 \mathrm{~min}$ (major).
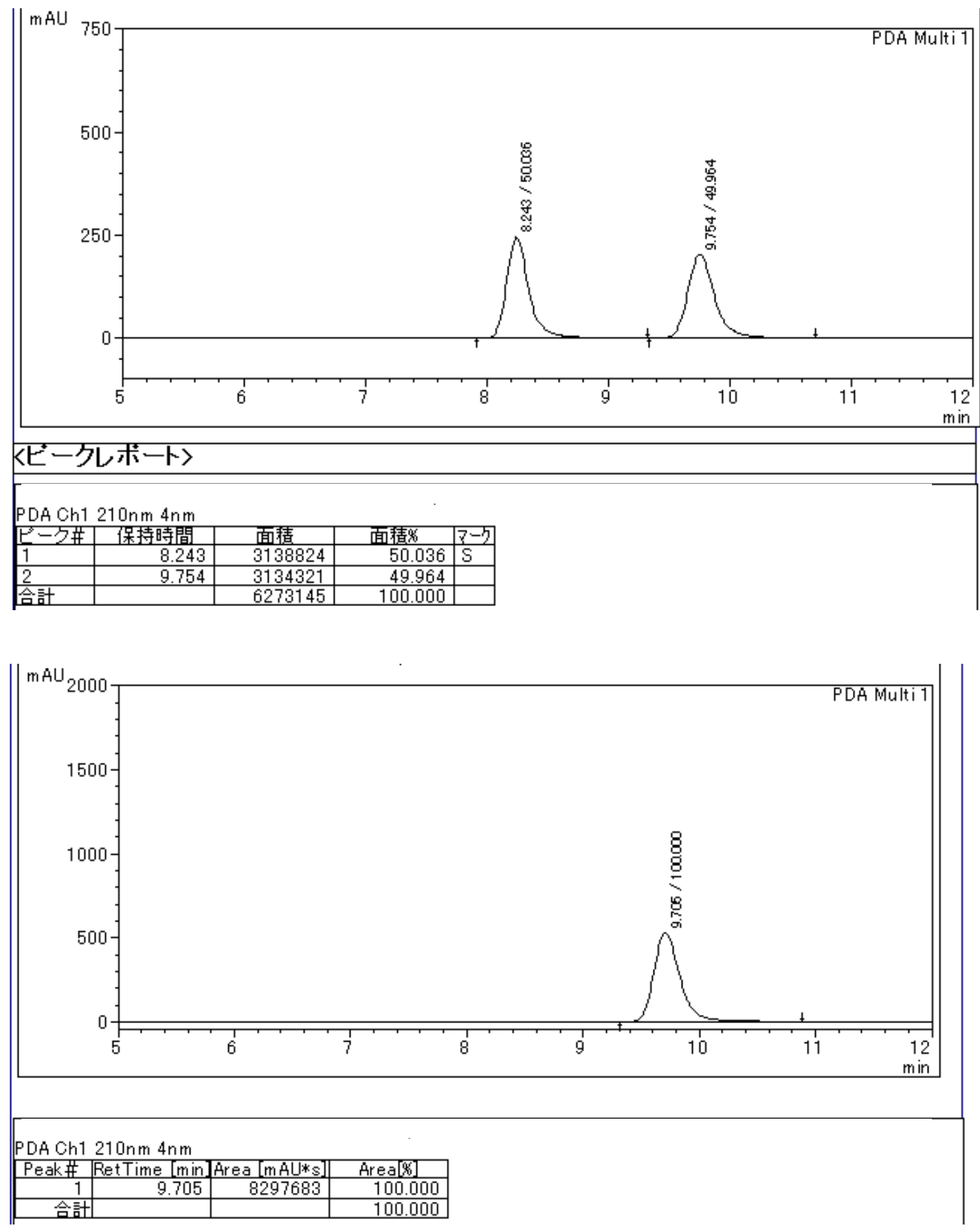


\section{(S)- $N$-(tert-Butyl(ethyl)(oxo)- $\lambda^{6}$-sulfanylidene)pivalamide (2a)}<smiles>CC[S@](=O)(CC(C)(C)C)NC(=O)C(C)(C)C</smiles>

HPLC analysis: Daicel CHIRALPAK IC, hexane/ $i \mathrm{PrOH}=1 / 1$, flow rate $0.75 \mathrm{~mL} / \mathrm{min}$, $\lambda=210 \mathrm{~nm}$, retention time: $8.1 \mathrm{~min}$ (major) and $9.6 \mathrm{~min}$ (minor).

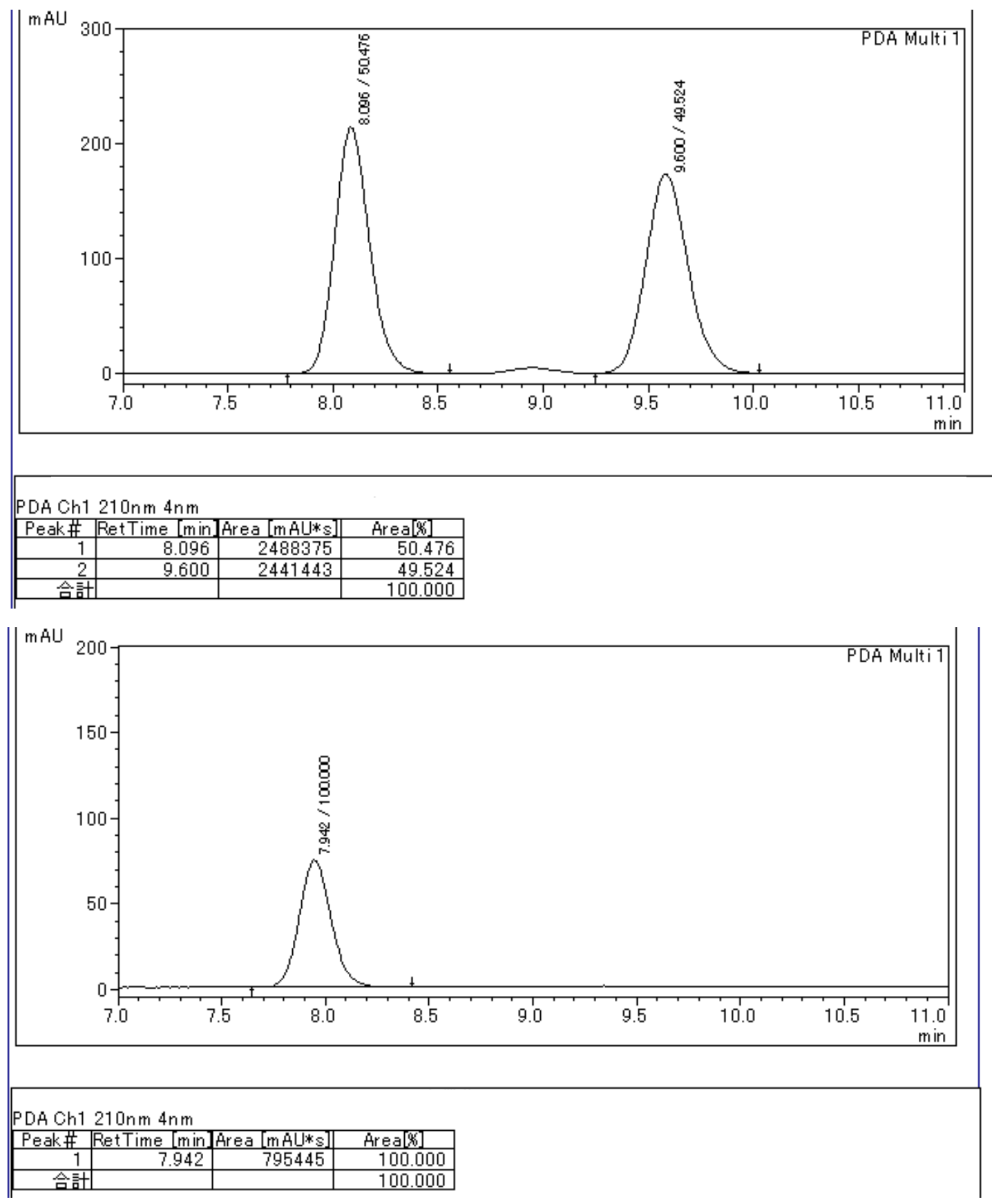


10 mmol scale reaction

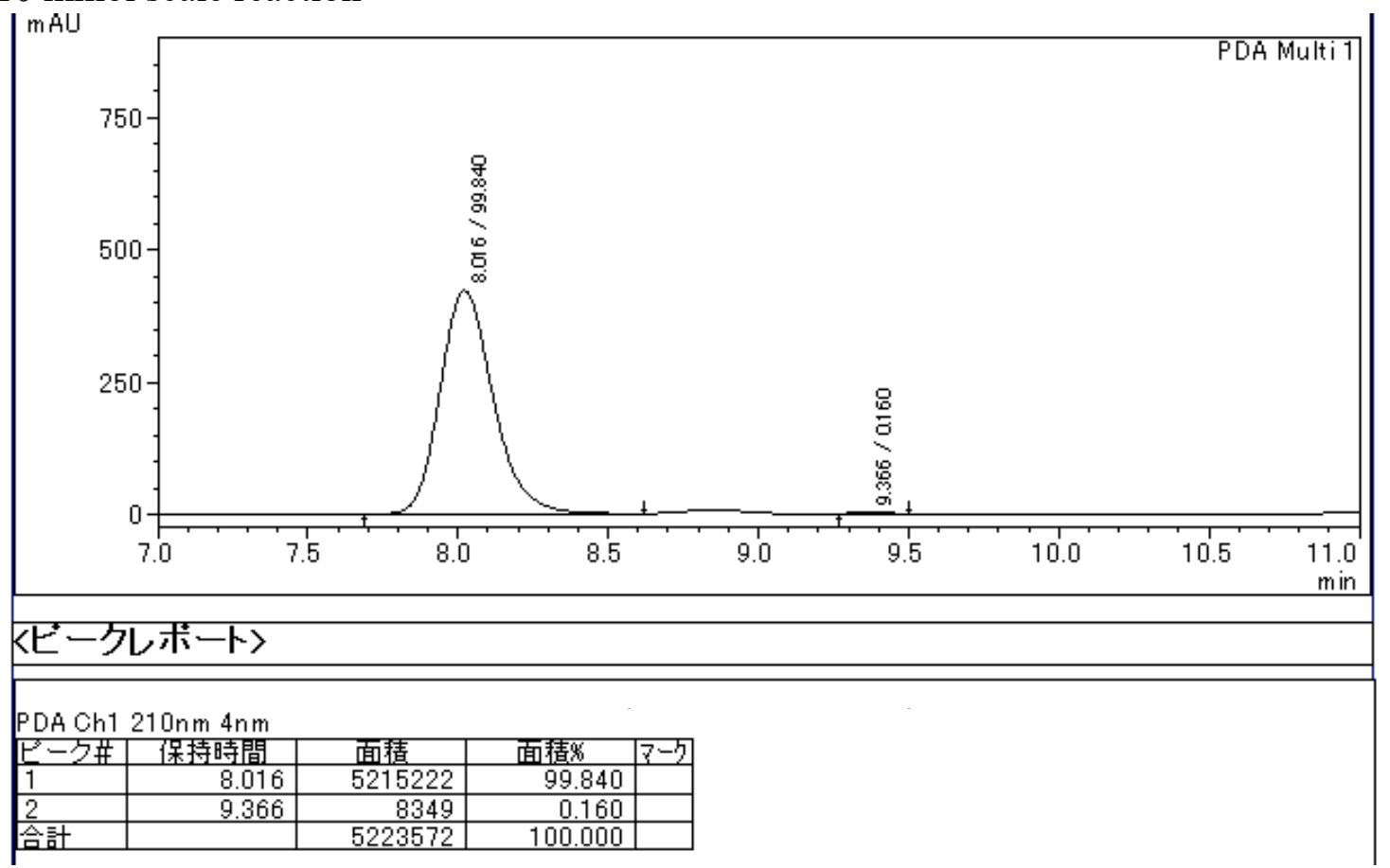


(R)-N-(tert-Butyl(methyl)(oxo)- $\lambda^{6}$-sulfanylidene)pivalamide (2b)<smiles>CC(C)(C)C(=O)N[S@](C)(=O)C(C)(C)C</smiles>

HPLC analysis: Daicel CHIRALPAK IC, hexane $/ i \mathrm{PrOH}=1 / 1$, flow rate $0.75 \mathrm{~mL} / \mathrm{min}, \lambda$ $=210 \mathrm{~nm}$, retention time: $9.8 \mathrm{~min}$ (minor) and $14.9 \mathrm{~min}$ (major).

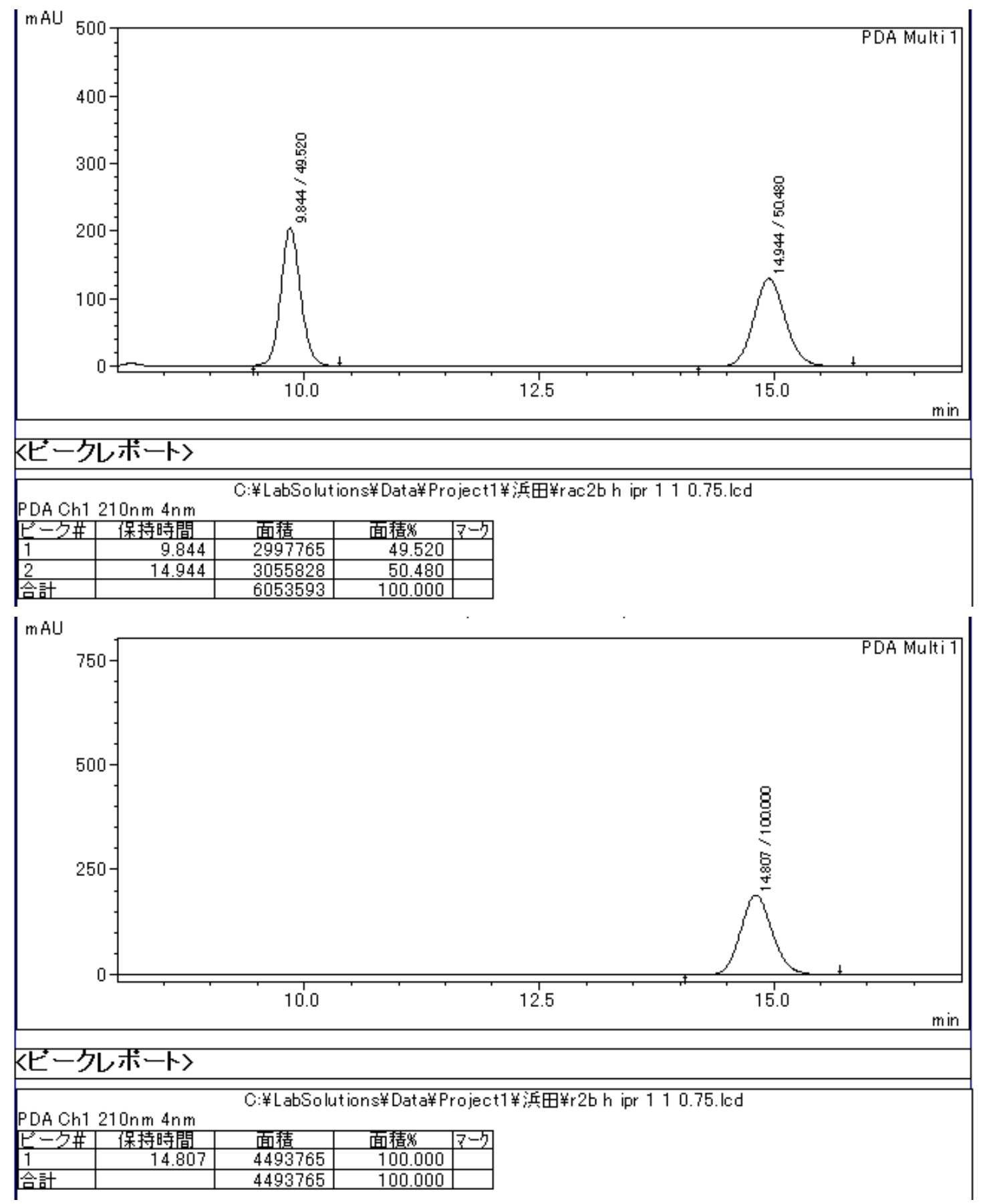




\section{(R)-N-(tert-Butyl(butyl)(oxo)- $\lambda^{6}$-sulfanylidene)pivalamide (2c)}<smiles>CC(C)(C)C(=O)N[Sb](=O)(Br)(C(C)(C)C)C(C)(C)C</smiles>

HPLC analysis: Daicel CHIRALPAK IC, hexane $/ \mathrm{PrOH}=1 / 1$, flow rate $0.75 \mathrm{~mL} / \mathrm{min}$, $\lambda=210 \mathrm{~nm}$, retention time: $6.6 \mathrm{~min}$ (minor) and $7.2 \mathrm{~min}$ (major).

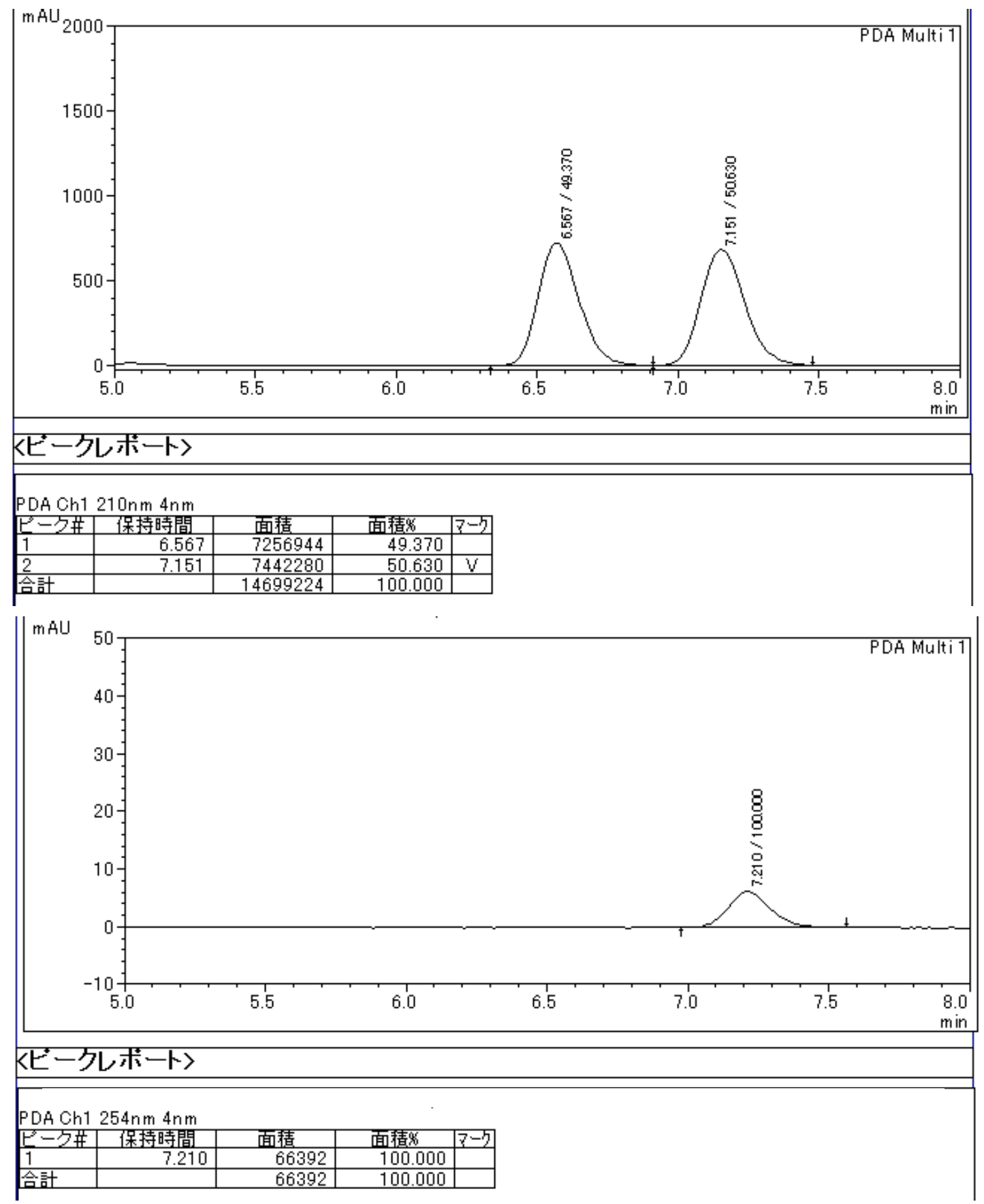


$(R)-N$-(tert-Buyl(hexyl)(oxo)- $\lambda^{6}$-sulfanylidene)pivalamide (2d)<smiles></smiles>

HPLC analysis: Daicel CHIRALPAK IC, hexane $/ \mathrm{PrOH}=3 / 1$, flow rate $0.75 \mathrm{~mL} / \mathrm{min}$, $\lambda=210 \mathrm{~nm}$, retention time: $9.2 \mathrm{~min}$ (minor) and $9.8 \mathrm{~min}$ (major).

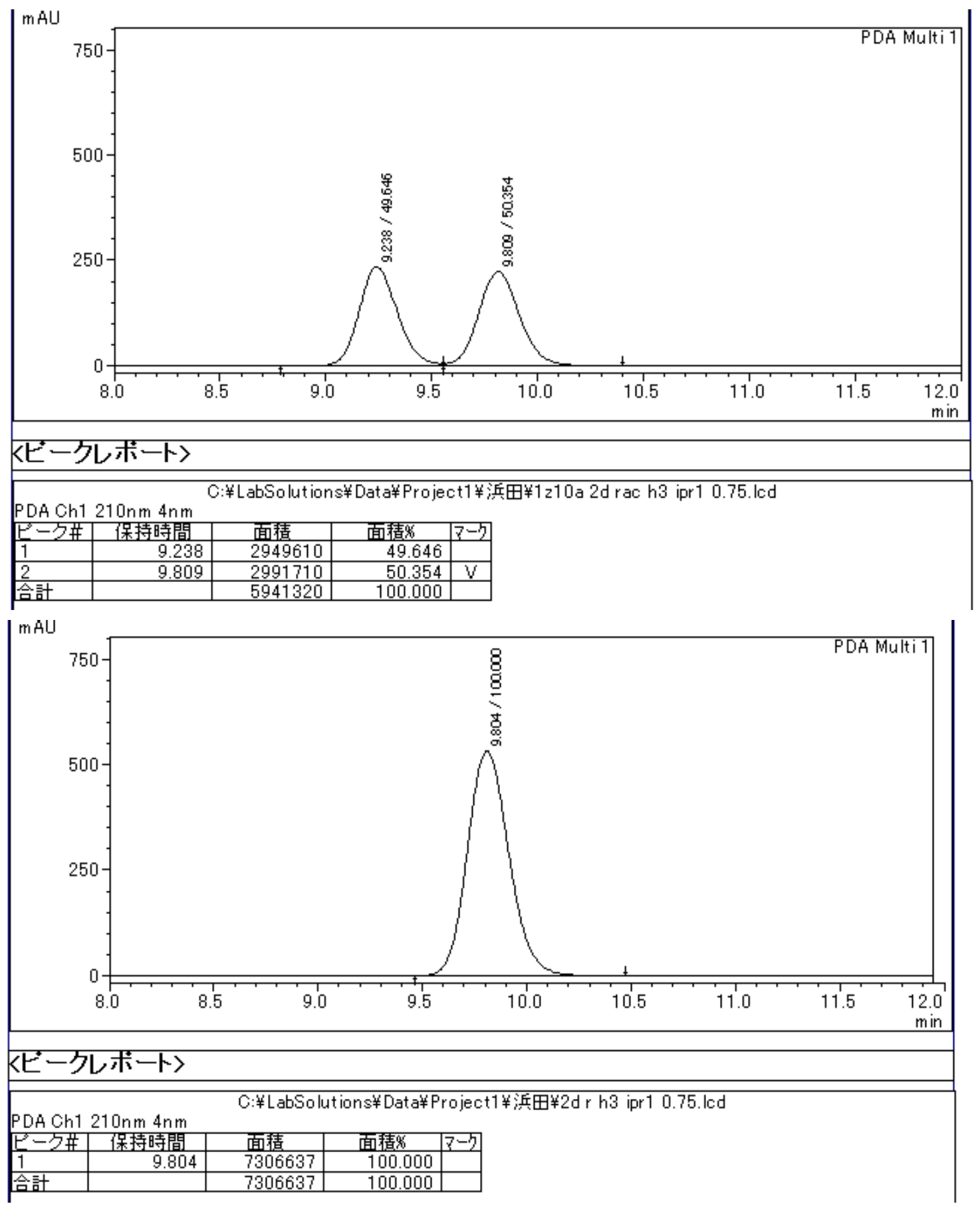


(R)-N-(tert-Butyl(isobutyl)(oxo)- $\lambda^{6}$-sulfanylidene)pivalamide (2e)<smiles>CC(C)(C)[13C](=O)N[13C](=O)[13C](C)(C)[13CH3]</smiles>

HPLC analysis: Daicel CHIRALPAK IC-3, hexane $/ \mathrm{PrOH}=50 / 1$, flow rate 0.75 $\mathrm{mL} / \mathrm{min}, \lambda=210 \mathrm{~nm}$, retention time: $53.4 \mathrm{~min}$ (minor) and $56.9 \mathrm{~min}$ (major).

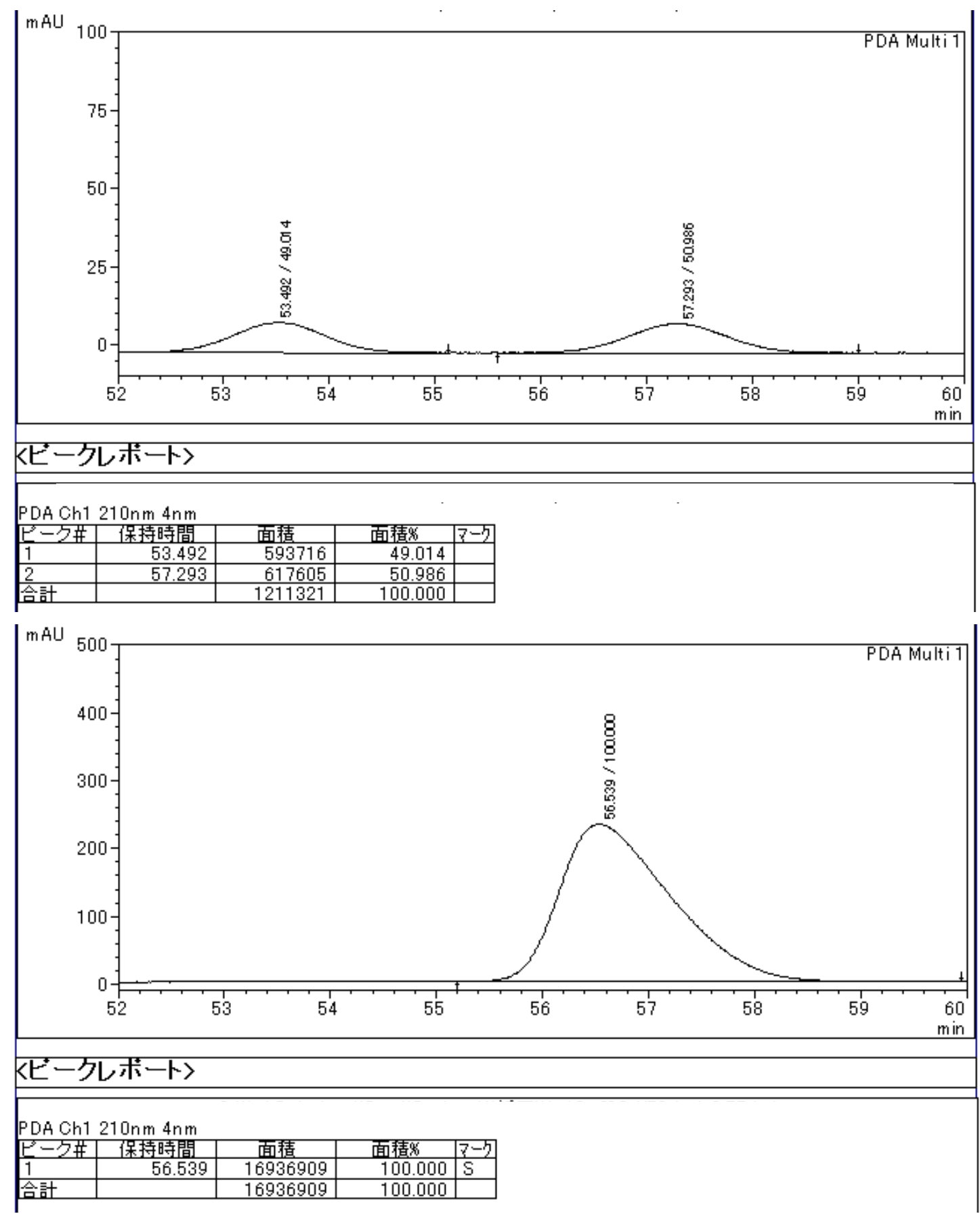


(R)- $N$-(tert-Butyl(oxo)(3-((triisopropylsilyl)oxy)propyl)- $\lambda^{6}$ sulfanylidene)pivalamide (2f) $(99 \%$ ee)

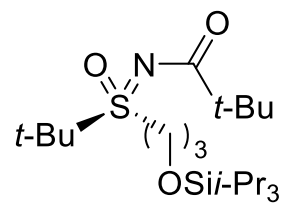

HPLC analysis: Daicel CHIRALPAK IC, hexane $/ \mathrm{PrOH}=3 / 1$, flow rate $0.75 \mathrm{~mL} / \mathrm{min}$, $\lambda=210 \mathrm{~nm}$, retention time: $7.5 \mathrm{~min}$ (minor) and $8.3 \mathrm{~min}$ (major).

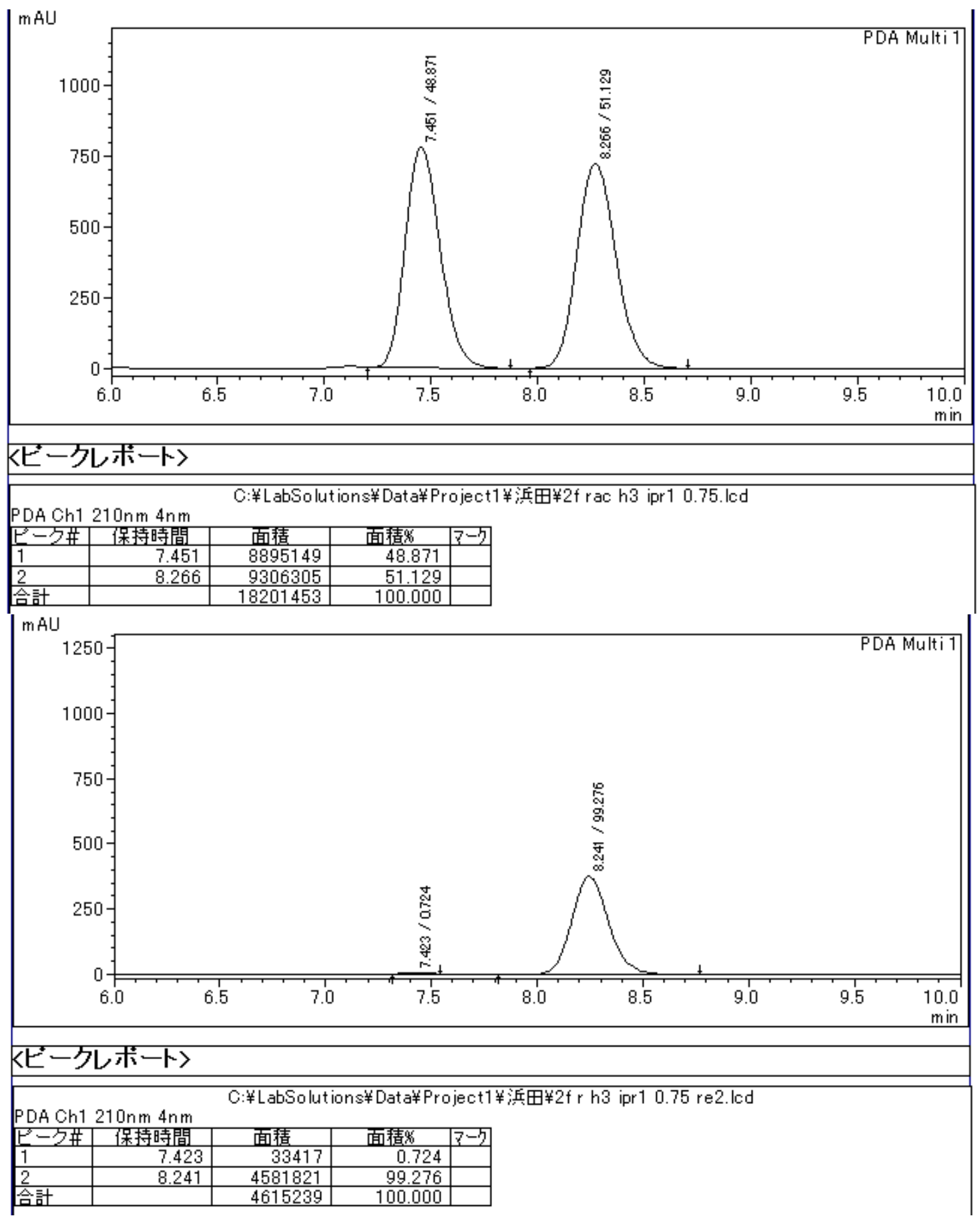


(R)- $N$-((4-(1,3-Dioxolan-2-yl)butyl)(tert-butyl)(oxo)- $\lambda^{6}$-sulfanylidene)pivalamide (2g)

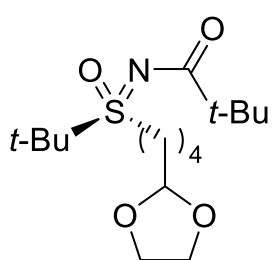

HPLC analysis: Daicel CHIRALPAK IC, hexane $/ \mathrm{PrOH}=1 / 1$, flow rate $0.75 \mathrm{~mL} / \mathrm{min}$, $\lambda=210 \mathrm{~nm}$, retention time: $11.9 \mathrm{~min}$ (major) and $12.9 \mathrm{~min}$ (minor).

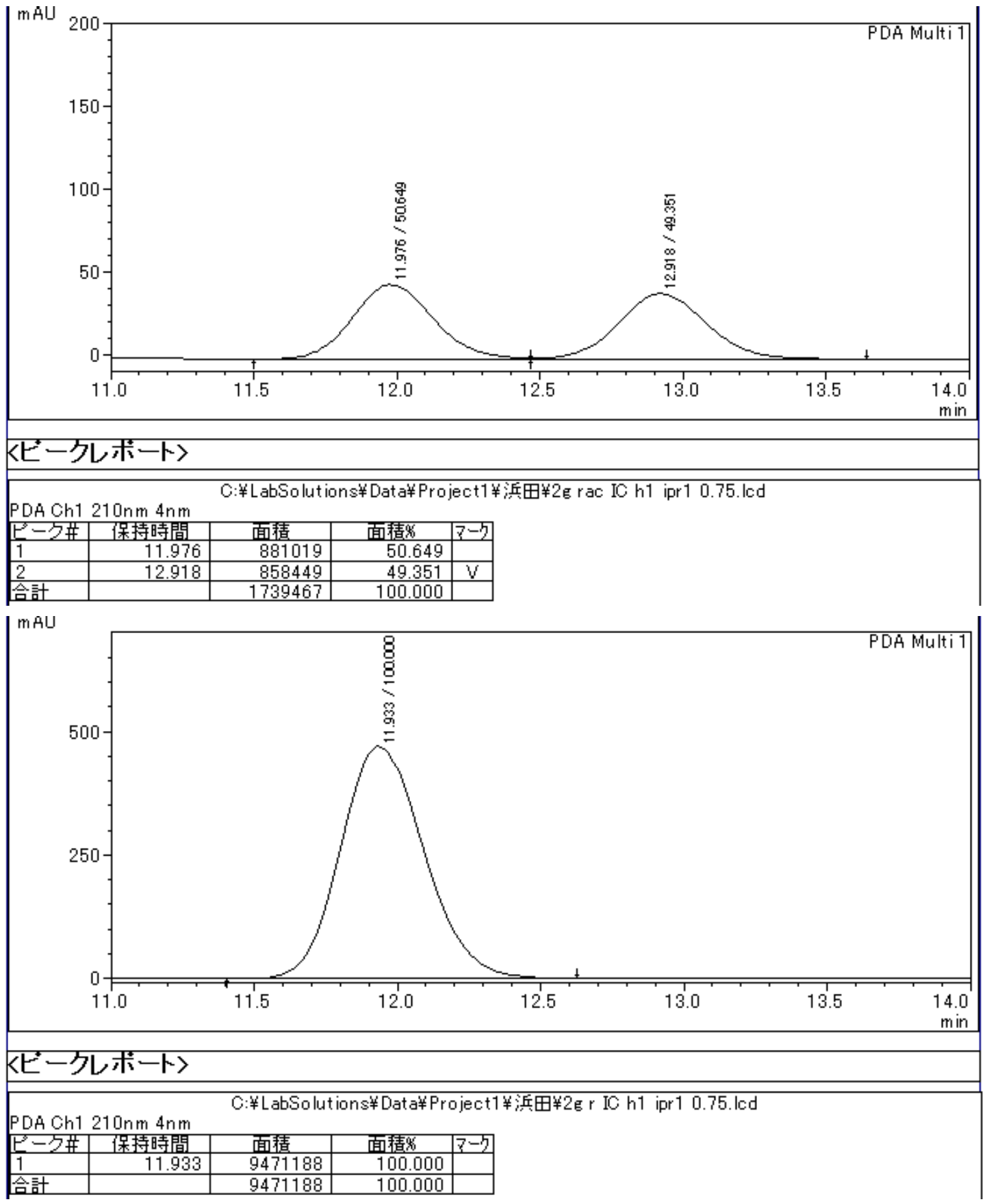


(R)-N-(tert-Butyl(5-iodopentyl)(oxo)- $\lambda^{6}$-sulfanylidene)pivalamide (2h)

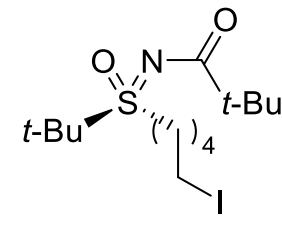

HPLC analysis: Daicel CHIRALPAK IC, hexane $/ \mathrm{PrOH}=10 / 1$, flow rate 0.75 $\mathrm{mL} / \mathrm{min}, \lambda=210 \mathrm{~nm}$, retention time: $28.5 \mathrm{~min}$ (minor) and $31.4 \mathrm{~min}$ (major).
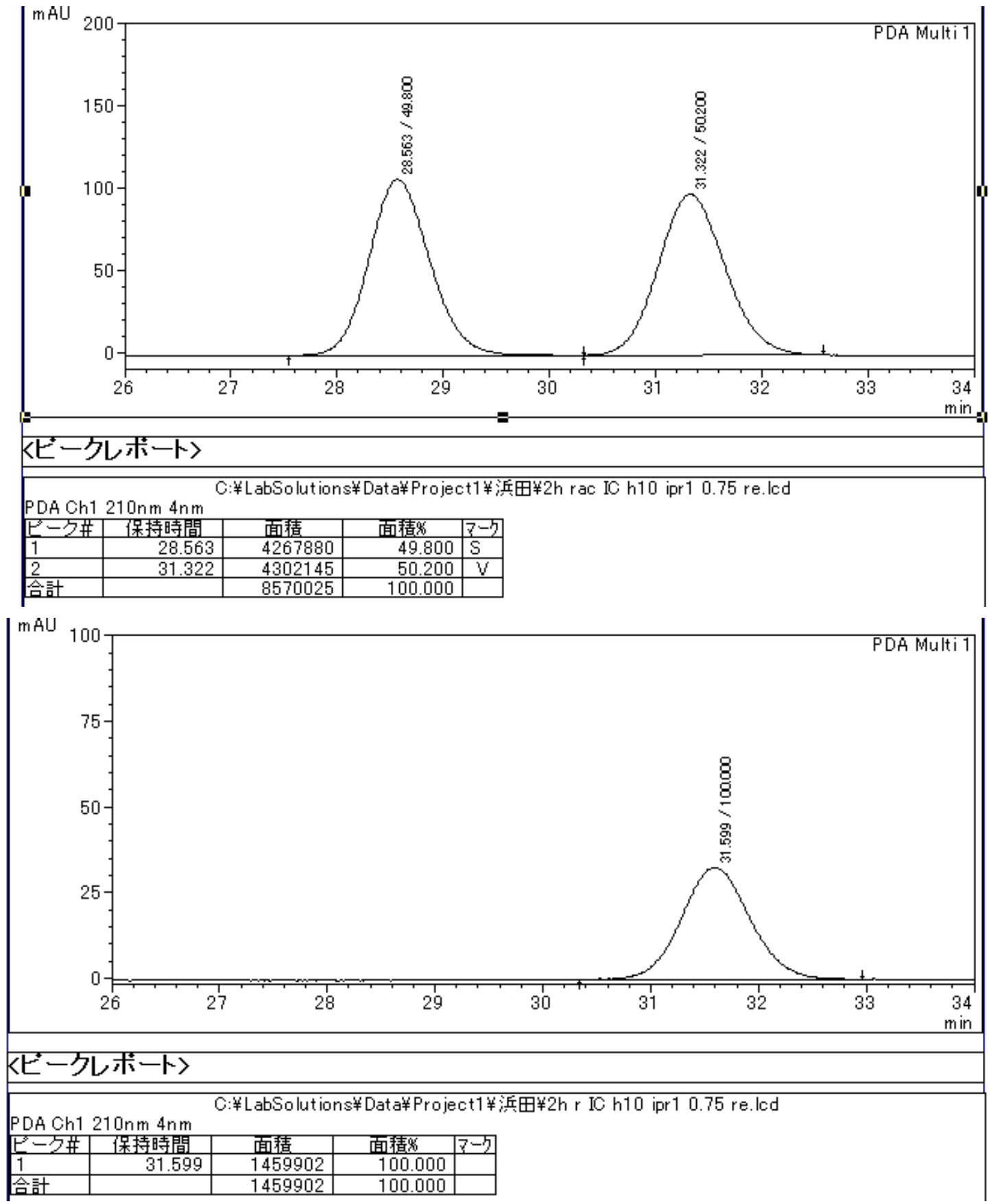
(R)- $N$-(Benzyl(tert-butyl)(oxo)- $\lambda^{6}$-sulfanylidene)pivalamide (2i)<smiles>CC(C)(C)C(=O)N[Sb](=O)(Br)(Br)C(C)(C)C</smiles>

HPLC analysis: Daicel CHIRALPAK IC-3, hexane $/ \mathrm{PrOH}=15 / 1$, flow rate 0.75 $\mathrm{mL} / \mathrm{min}, \lambda=210 \mathrm{~nm}$, retention time: $105.0 \mathrm{~min}$ (major) and $119.1 \mathrm{~min}$ (minor).

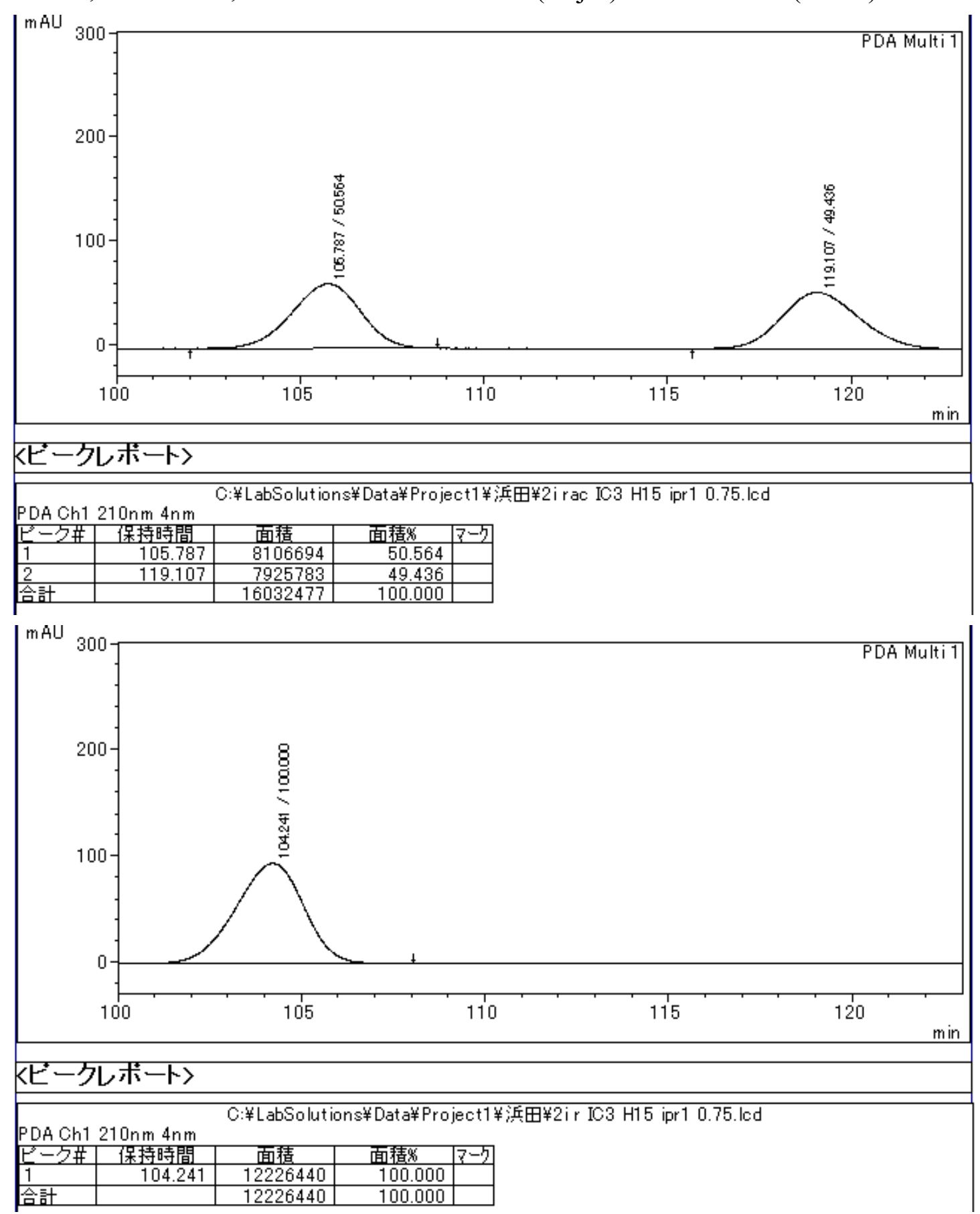


(R)-N-(tert-Butyl(allyl)(oxo)- $\lambda^{6}$-sulfanylidene)pivalamide (2j)<smiles>C=CC[SH](=O)(CC(C)(C)C)NC(=O)C(C)(C)C</smiles>

HPLC analysis: Daicel CHIRALPAK IC, hexane $/ \mathrm{PrOH}=1 / 1$, flow rate $0.75 \mathrm{~mL} / \mathrm{min}$, $\lambda=210 \mathrm{~nm}$, retention time: $8.5 \mathrm{~min}$ (minor) and $10.7 \mathrm{~min}$ (major).
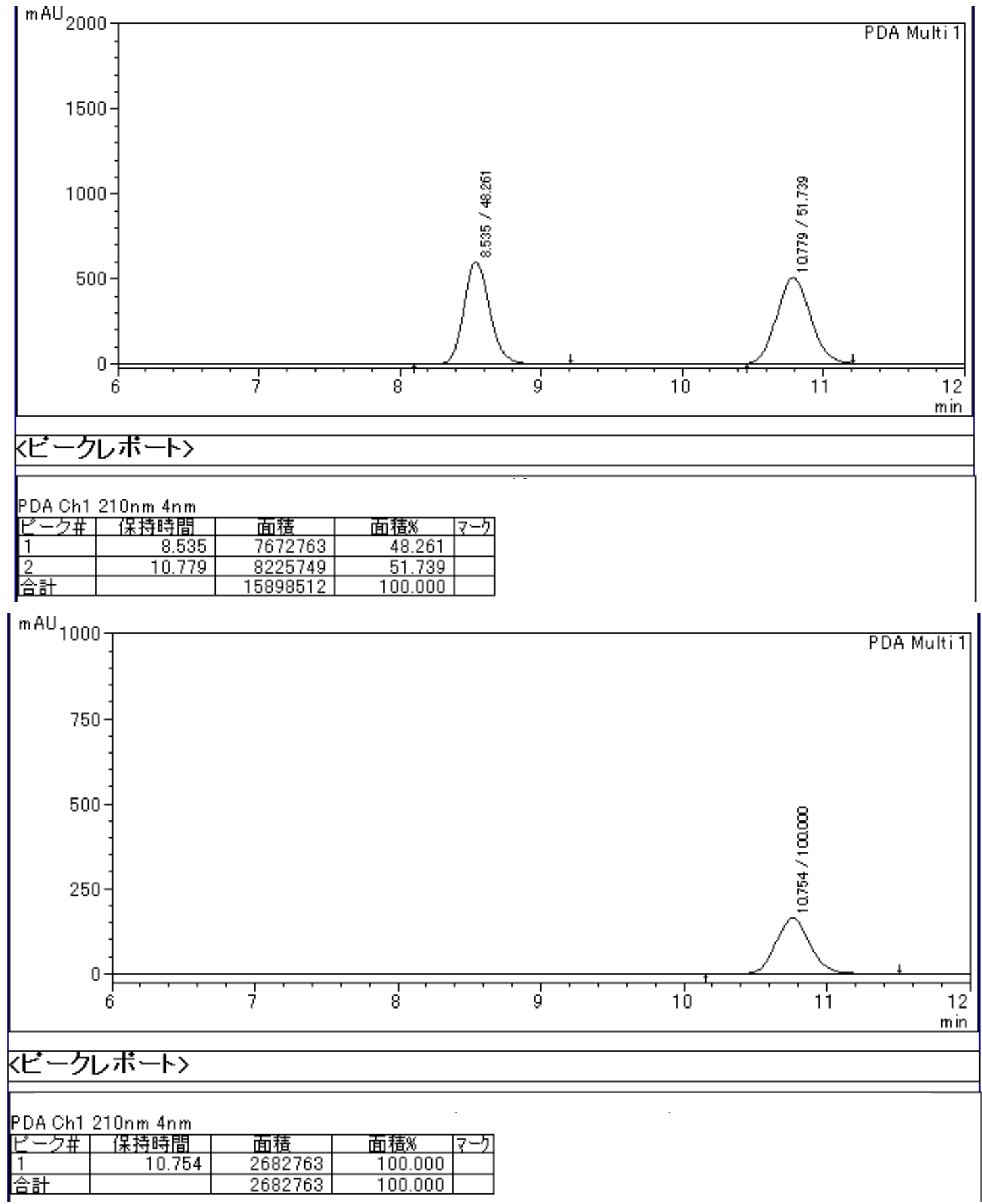
$(R, E)-N$-(But-2-en-1-yl(tert-butyl)(oxo)- $\lambda^{6}$-sulfanylidene)pivalamide (2k)<smiles>CC=CCS(=O)(=O)NC(=O)C(C)(C)C</smiles>

(E)-isomer

HPLC analysis: Daicel CHIRALPAK IC-3, hexane $/ \mathrm{iPrOH}=3 / 1$, flow rate 0.75 $\mathrm{mL} / \mathrm{min}, \lambda=210 \mathrm{~nm}$, retention time: $18.5 \mathrm{~min}$ (minor) and $22.5 \mathrm{~min}$ (major).

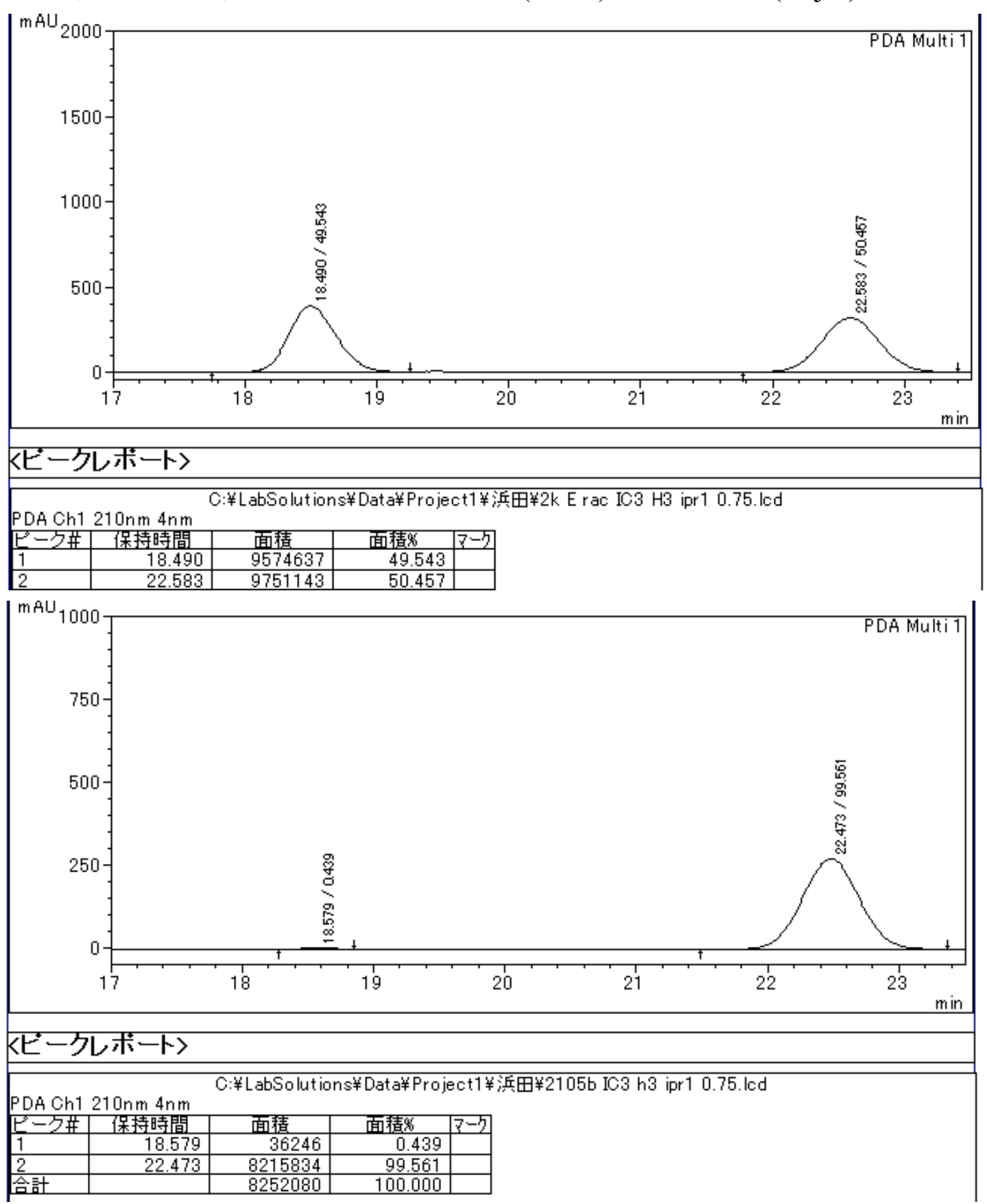


(Z)-isomer

HPLC analysis: Daicel CHIRALPAK IC-3, hexane $/ \mathrm{PrOH}=3 / 1$, flow rate 0.75

$\mathrm{mL} / \mathrm{min}, \lambda=210 \mathrm{~nm}$, retention time: $19.3 \mathrm{~min}$ (minor) and $23.7 \mathrm{~min}$ (major).

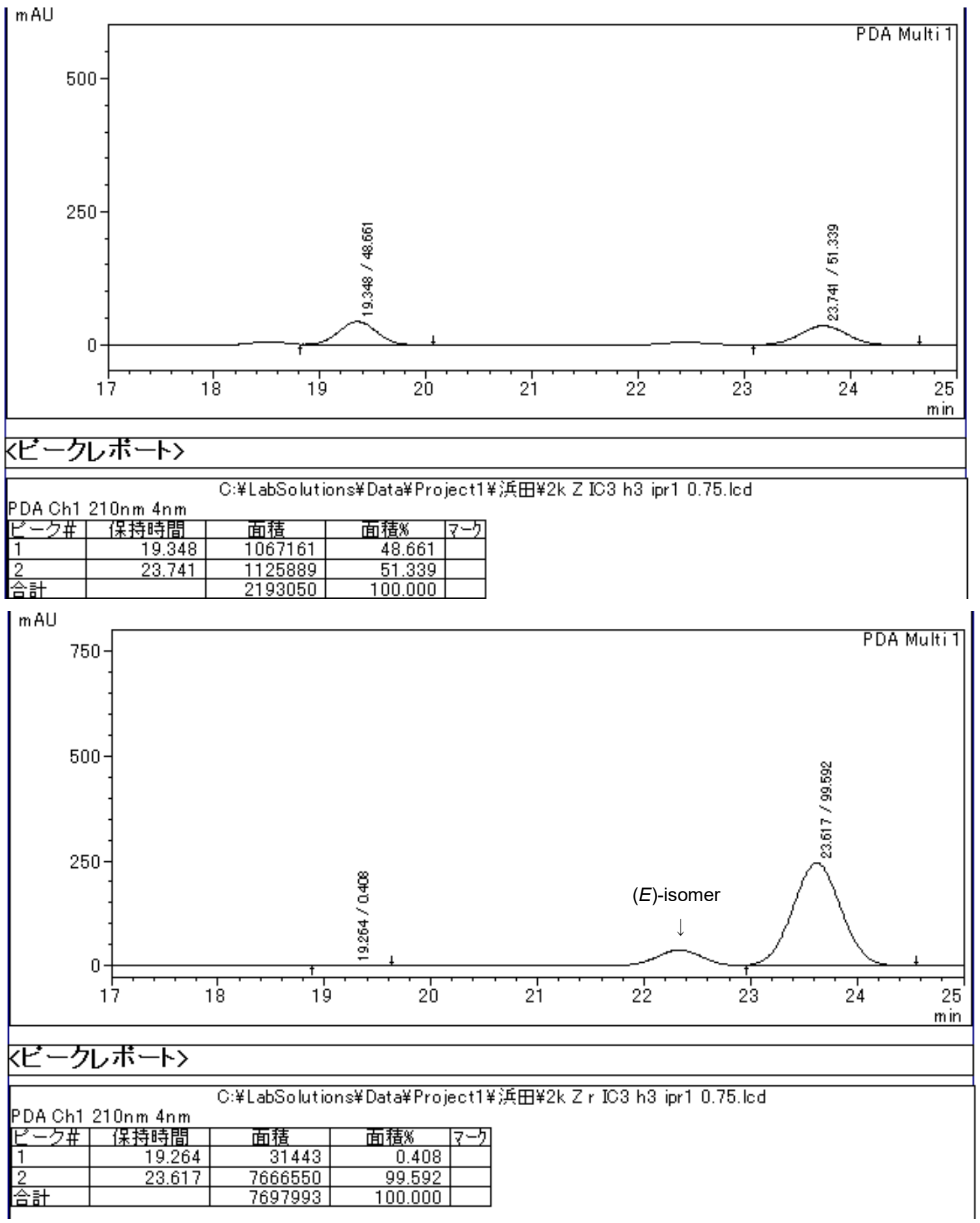


$(R, E)-N$-(3-Phenylprop-2-en-1-yl(tert-butyl)(oxo)- $-\lambda^{6}$-sulfanylidene)pivalamide (2I)<smiles>CC(C)(C)[Mg][SH](=O)(C/C=C/c1ccccc1)NC(=O)C(C)(C)C</smiles>

HPLC analysis: Daicel CHIRALPAK IC-3, hexane $/ \mathrm{iPrOH}=5 / 1$, flow rate 0.75 $\mathrm{mL} / \mathrm{min}, \lambda=210 \mathrm{~nm}$, retention time: $39.7 \mathrm{~min}$ (major) and $44.6 \mathrm{~min}$ (minor).

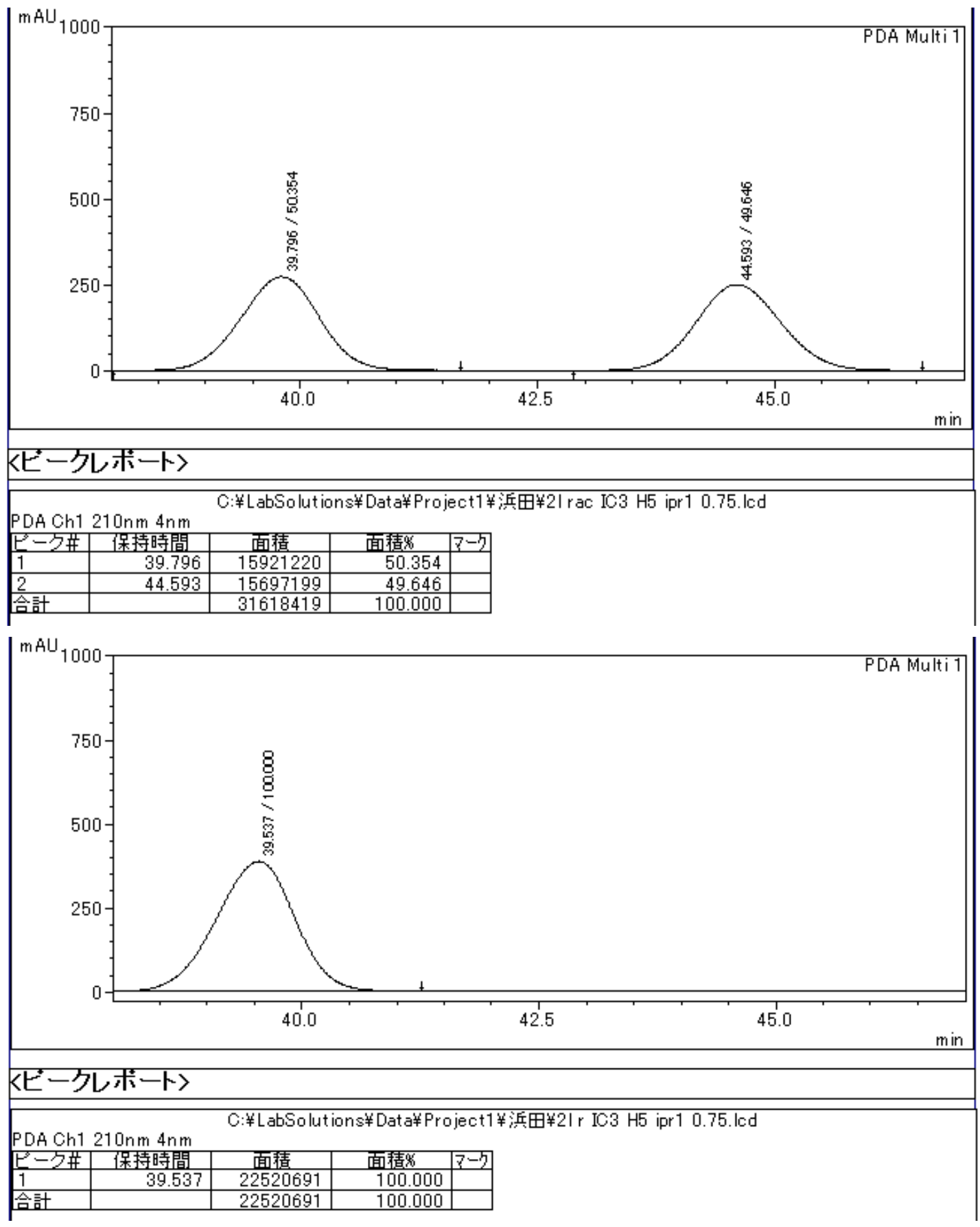


(R)- $N$-(2-Methylprop-2-en-1-yl(tert-butyl)(oxo)- $\lambda^{6}$-sulfanylidene)pivalamide (2m)<smiles>C=C(C)CS(=O)(=O)NC(=O)C(C)(C)C</smiles>

HPLC analysis: Daicel CHIRALPAK IC, hexane $/ \mathrm{PrOH}=3 / 1$, flow rate $0.75 \mathrm{~mL} / \mathrm{min}$, $\lambda=210 \mathrm{~nm}$, retention time: $11.4 \mathrm{~min}$ (minor) and $17.0 \mathrm{~min}$ (major).
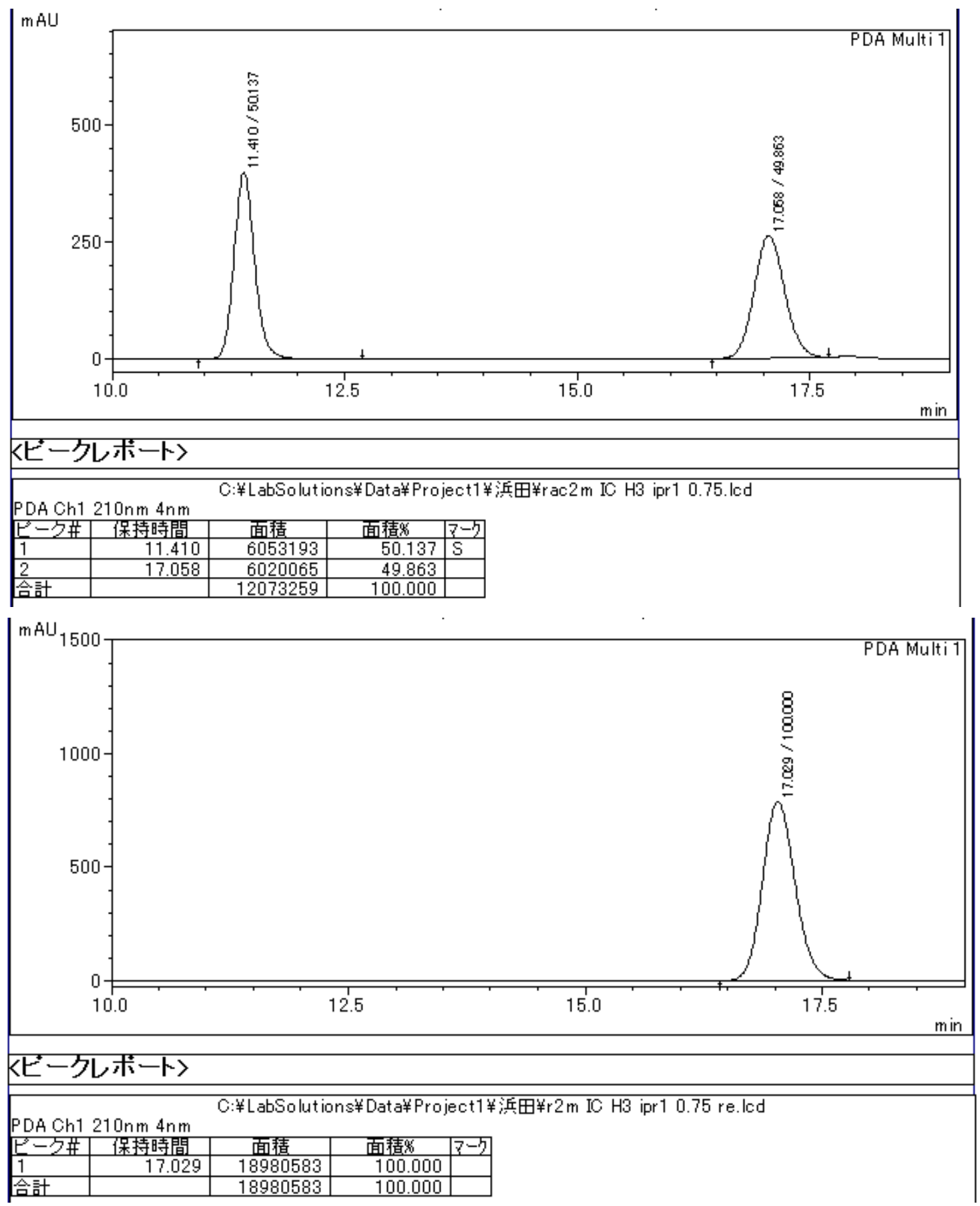
$(R, E)-N$-(2-Methylbut-2-en-1-yl(tert-butyl)(oxo)- $\lambda^{6}$-sulfanylidene)pivalamide (2n)<smiles>CC=C(C)CS(=O)(=O)NC(=O)C(C)(C)C</smiles>

HPLC analysis: Daicel CHIRALPAK IC-3, hexane $/ \mathrm{iPrOH}=2 / 1$, flow rate 0.75 $\mathrm{mL} / \mathrm{min}, \lambda=210 \mathrm{~nm}$, retention time: $11.8 \mathrm{~min}$ (minor) and $16.5 \mathrm{~min}$ (major).
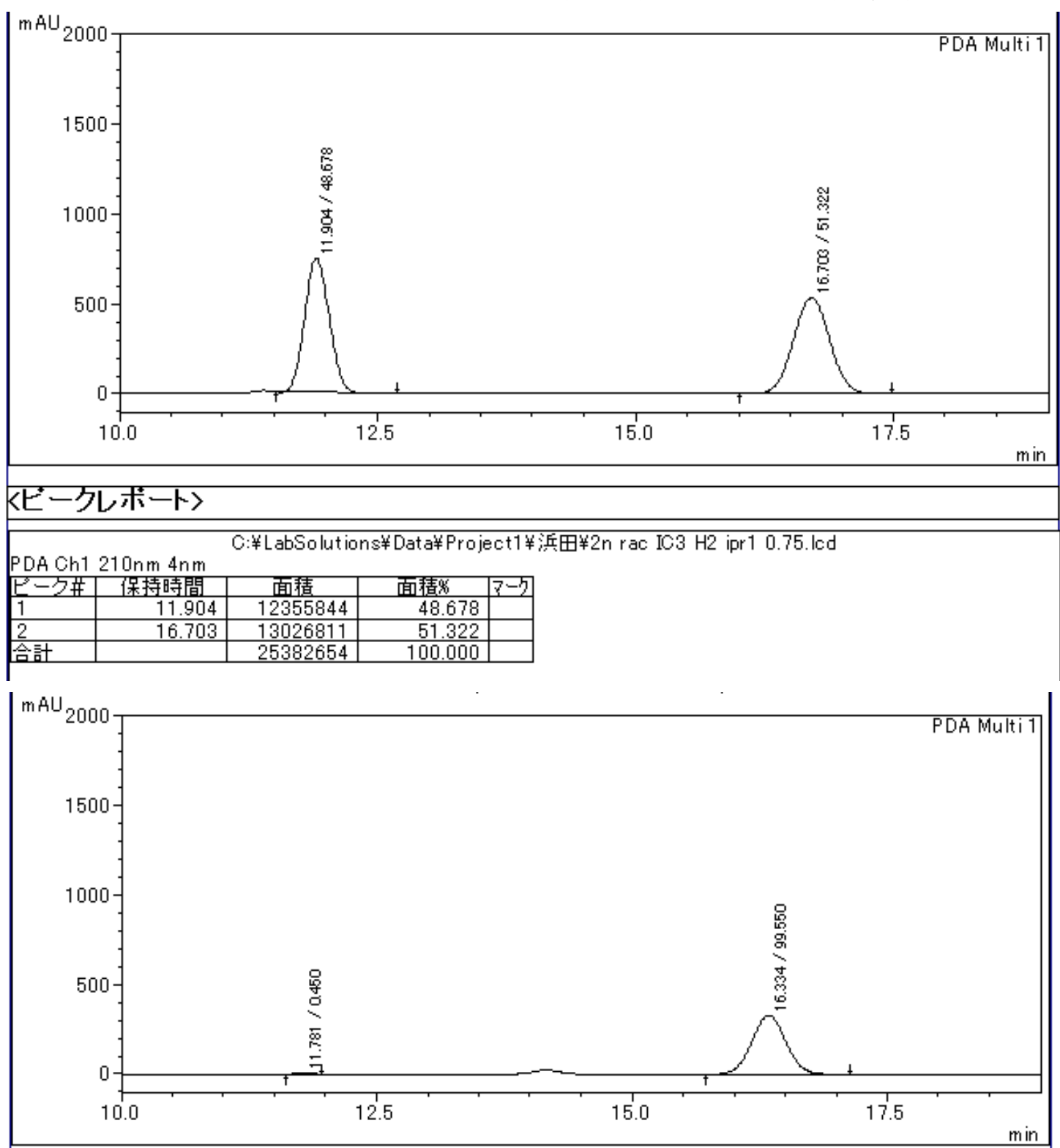

\begin{tabular}{|c|c|c|c|}
\hline 《ビーろ & しポート〉 & & \\
\hline PDA Ch1 & $210 \mathrm{~nm} 4 \mathrm{~nm}$ & :LabSoluti & 羊ata羊Project1 1 羊 \\
\hline$e^{2}-3 \#$ & 保持時間 & 面猜 & 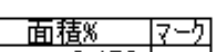 \\
\hline & 11.781 & $\frac{34873}{371012}$ & \begin{tabular}{|l|l|}
0.450 & \\
00550
\end{tabular} \\
\hline 会計 & & $\begin{array}{ll}712042 \\
7746915\end{array}$ & \begin{tabular}{r|r}
9900000 & \\
100.000 &
\end{tabular} \\
\hline
\end{tabular}


(R)- $N$-(But-2-yn-1-yl(tert-butyl)(oxo)- $\lambda^{6}$-sulfanylidene)pivalamide (2o)<smiles>CC#CCS(=O)(=NC(=O)C(C)(C)C)C(C)(C)C</smiles>

HPLC analysis: Daicel CHIRALPAK IC-3, hexane $/ i \mathrm{PrOH}=3 / 1$, flow rate 0.75 $\mathrm{mL} / \mathrm{min}, \lambda=210 \mathrm{~nm}$, retention time: $34.7 \mathrm{~min}$ (minor) and $56.8 \mathrm{~min}$ (major).
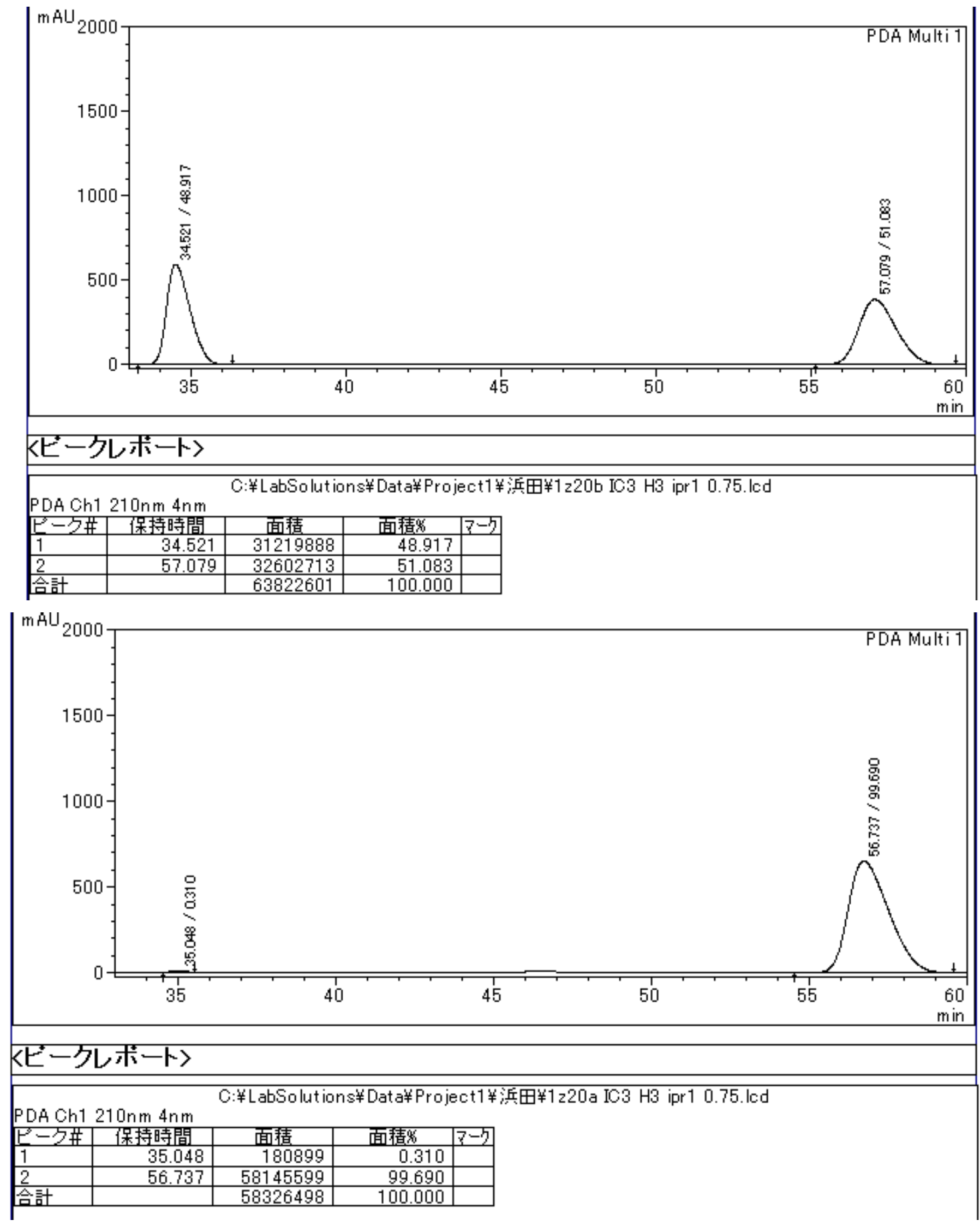
(R)-N-(tert-Butyl(2-oxobutyl)(oxo)- $\lambda^{6}$-sulfanylidene)pivalamide (2p)<smiles>CCC(=O)CS(=O)(=O)NC(=O)C(C)(C)C</smiles>

HPLC analysis: Daicel CHIRALPAK IC-3, hexane $/ \mathrm{PrOH}=3 / 1$, flow rate 0.75 $\mathrm{mL} / \mathrm{min}, \lambda=210 \mathrm{~nm}$, retention time: $8.4 \mathrm{~min}$ (major) and $9.0 \mathrm{~min}$ (minor).

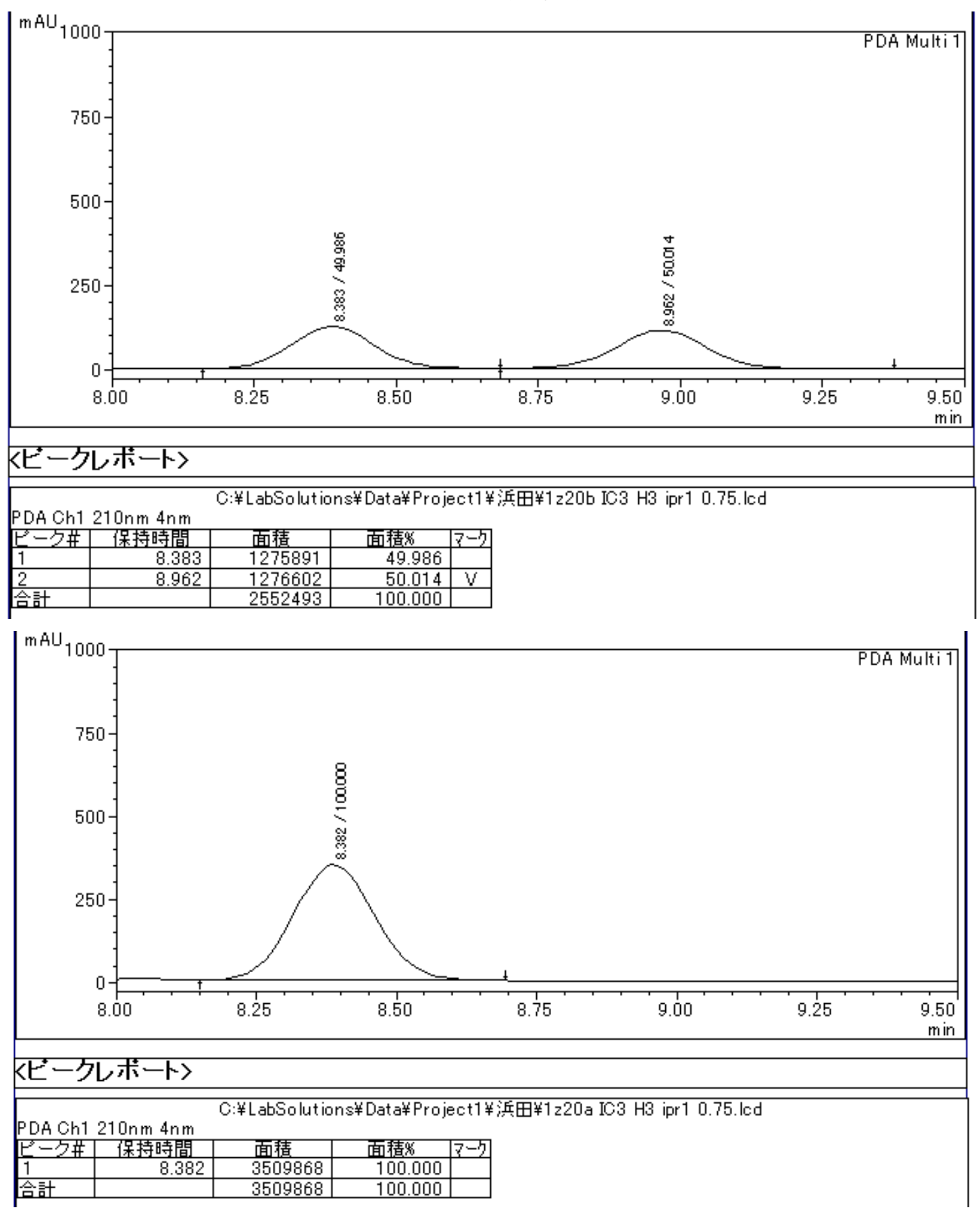


(R)- $N$-(tert-Butyl(2-oxo-3-phenylpropyl)(oxo)- $\lambda^{6}$-sulfanylidene)pivalamide (2q)<smiles>CC(C)(C)C(=O)NS(=O)(=O)CC(=O)Cc1ccccc1</smiles>

HPLC analysis: Daicel CHIRALPAK IC, hexane $/ \mathrm{PrOH}=15 / 1$, flow rate 0.75 $\mathrm{mL} / \mathrm{min}, \lambda=210 \mathrm{~nm}$, retention time: $19.7 \mathrm{~min}$ (minor) and $21.1 \mathrm{~min}$ (major).

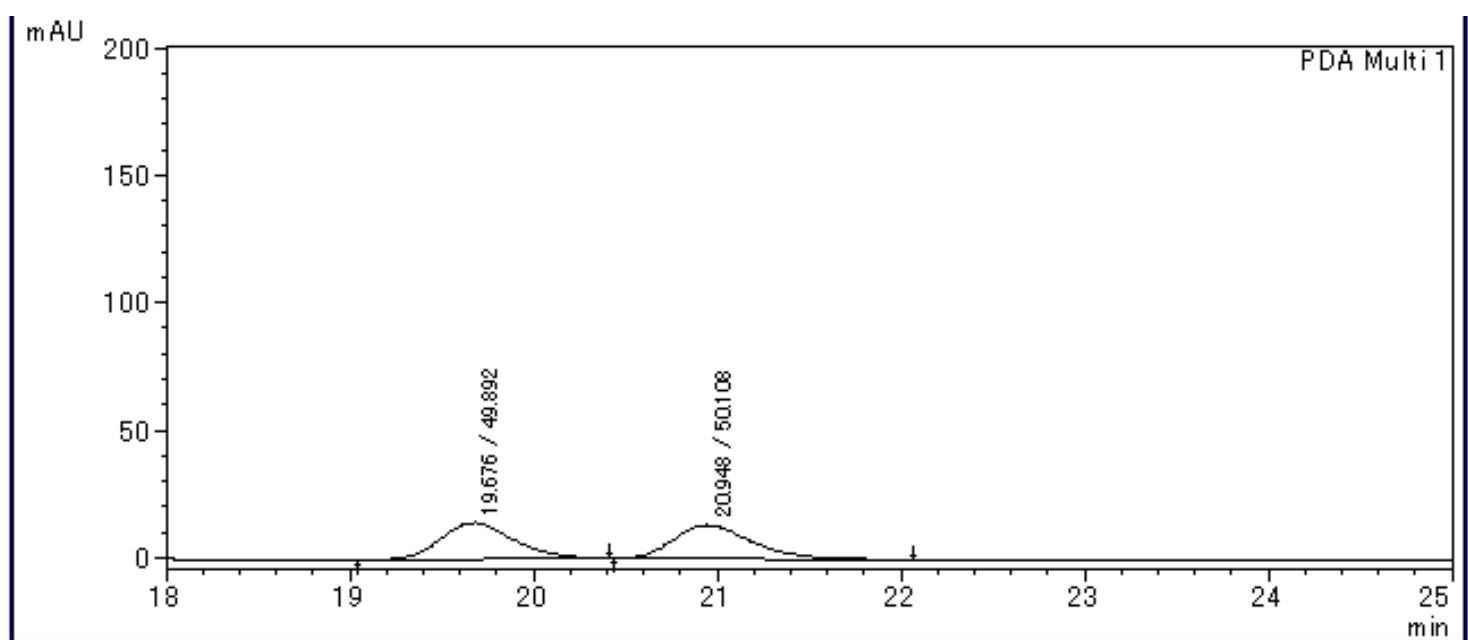

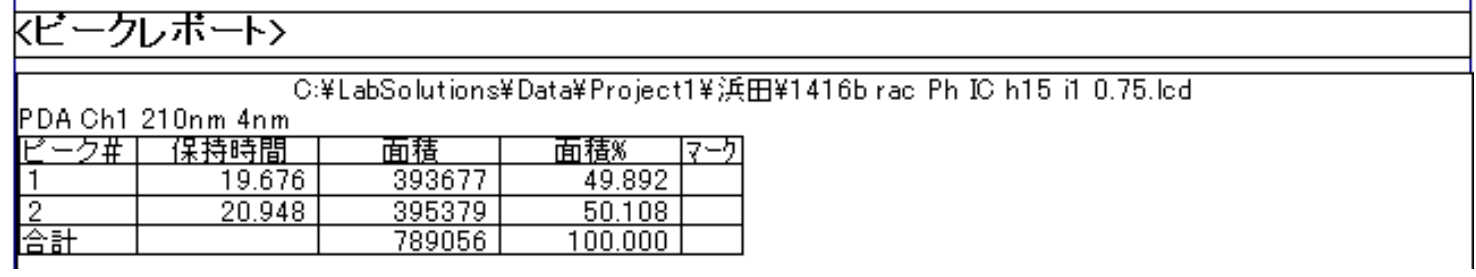

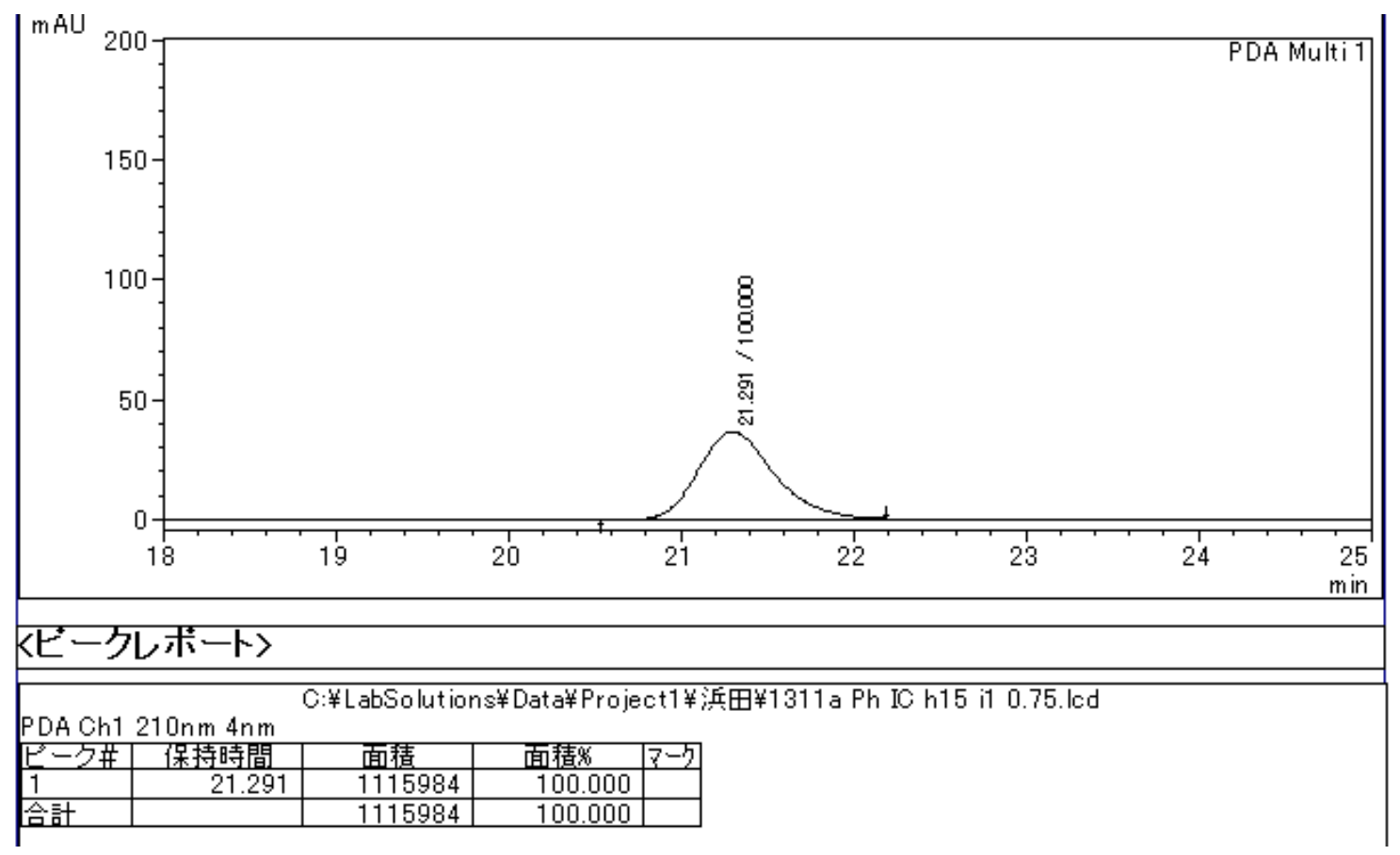


(S)-N-(tert-Butyl(ethoxy)- $\lambda^{4}$-sulfanylidene)pivalamide (5)<smiles>CCOS(=NC(=O)C(C)(C)C)C(C)(C)C</smiles>

HPLC analysis: Daicel CHIRALPAK IG, hexane $/ \mathrm{PrOH}=10 / 1$, flow rate 0.75 $\mathrm{mL} / \mathrm{min}, \lambda=254 \mathrm{~nm}$, retention time: $6.0 \mathrm{~min}$ (minor) and $6.5 \mathrm{~min}$ (major).

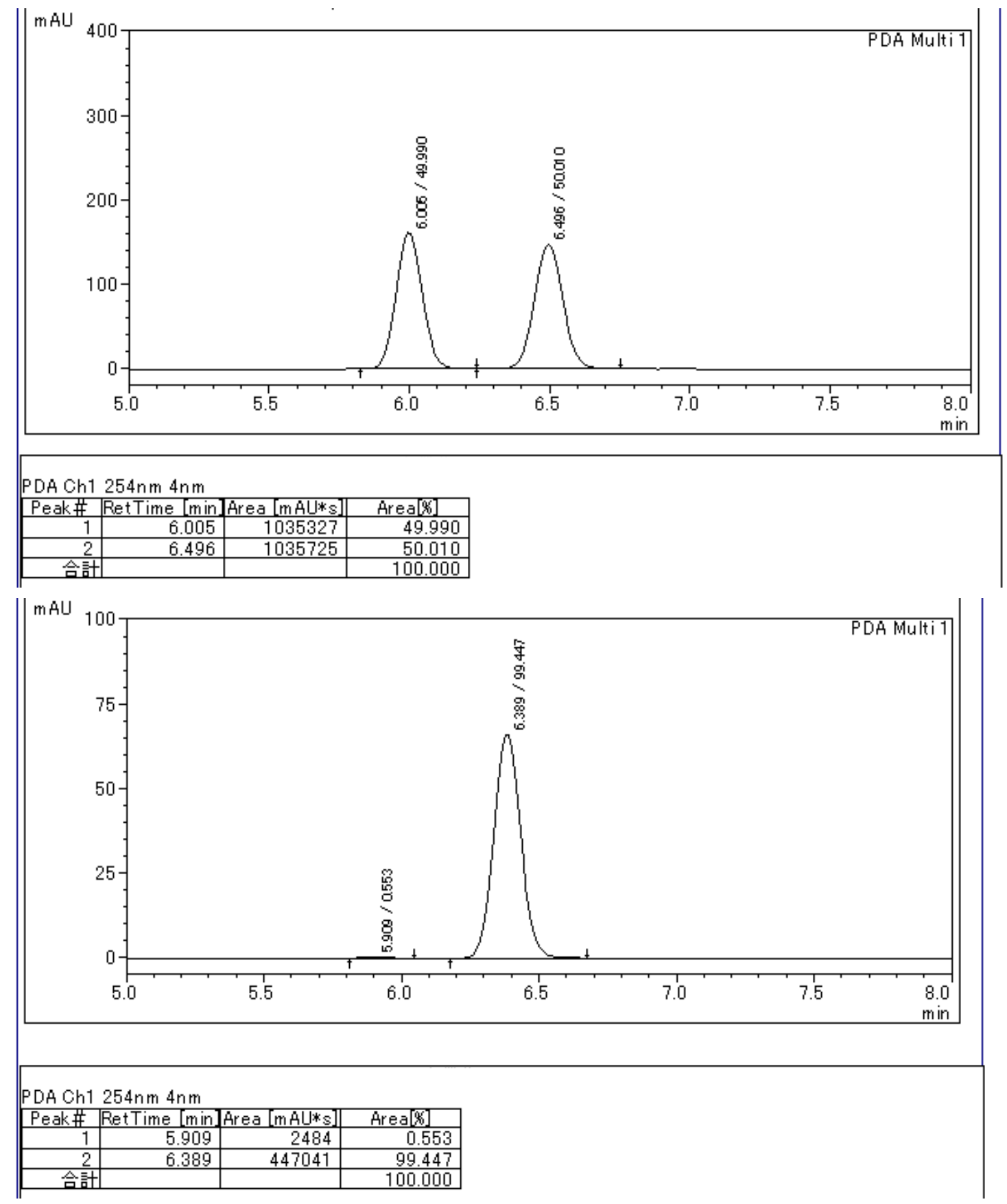


$(R, E)-N$-(tert-Butyl(oxo)(prop-1-en-1-yl)- $\lambda^{6}$-sulfanylidene)pivalamide (6j)<smiles>C/C=C/S(=O)(=NC(=O)C(C)(C)C)C(C)(C)C</smiles>

HPLC analysis: Daicel CHIRALPAK IC, hexane $/ i \mathrm{PrOH}=1 / 1$, flow rate $0.75 \mathrm{~mL} / \mathrm{min}$, $\lambda=210 \mathrm{~nm}$, retention time: $12.8 \mathrm{~min}$ (major) and $14.9 \mathrm{~min}$ (minor).

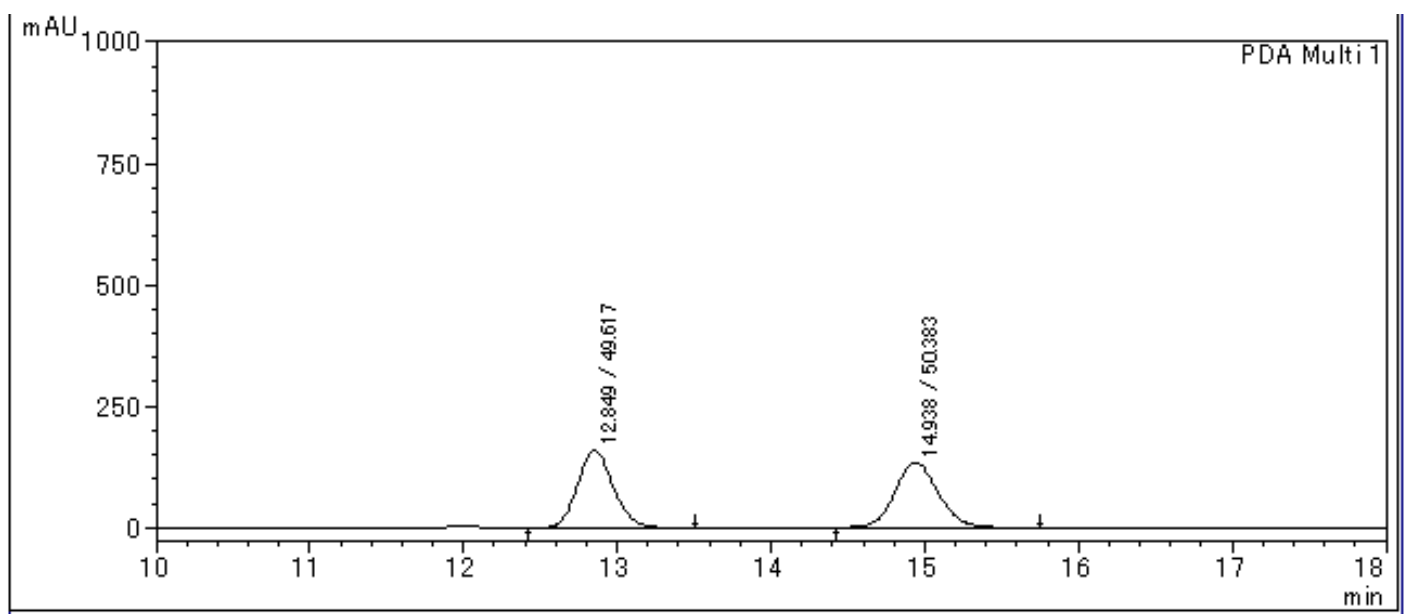

\begin{tabular}{|c|c|c|c|}
\hline \multicolumn{4}{|c|}{ 〈ビークレボート〉 } \\
\hline \multicolumn{4}{|c|}{$\begin{array}{l}\text { PDA Ch1 } 210 \mathrm{~nm} 4 \mathrm{~nm} \\
\mathrm{P}^{2}-\mathrm{CH}\end{array}$} \\
\hline$\frac{e^{2}-2 \#}{1}$ & 保持時間 & & \begin{tabular}{l|l|} 
面皘策 & $7-2$ \\
\end{tabular} \\
\hline & $\frac{12.849}{14.938}$ & $\frac{2575952}{2615769}$ & \begin{tabular}{|l|l|}
49.617 & \\
50.383 &
\end{tabular} \\
\hline 合計 & & 5191721 & \begin{tabular}{r|r}
50.0000 \\
100.000
\end{tabular} \\
\hline
\end{tabular}

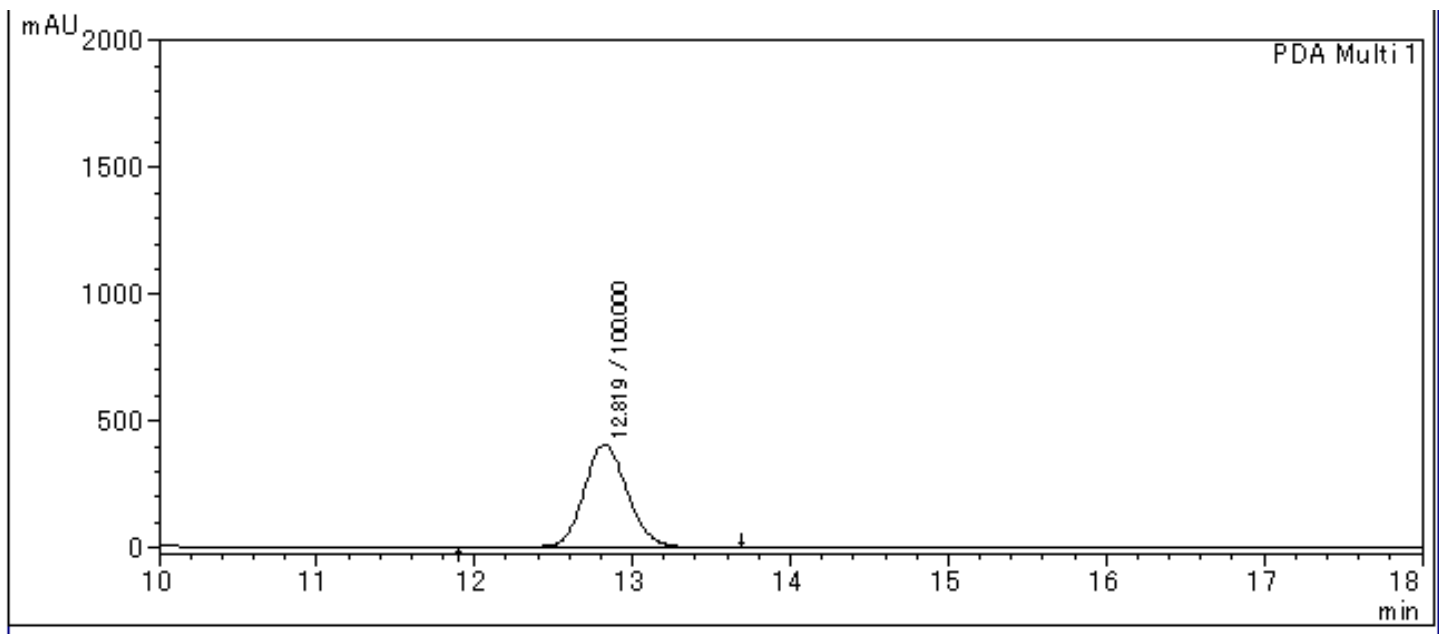

〈ビークしボート〉

\begin{tabular}{|c|c|c|c|c|}
\hline$F^{2}-h \#$ & 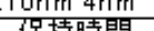 & & & \\
\hline 1 & $\frac{12.819}{12.98019}$ & $\frac{\text { 迤值 }}{7846498}$ & $\frac{1 \ln 100}{100000}$ & $x^{r}-2$ \\
\hline$\triangle \equiv+$ & & 7846498 & 100.000 & \\
\hline
\end{tabular}


(R)-N-(tert-Butyl(oxo)(2-methylprop-1-en-1-yl)- $\lambda^{6}$-sulfanylidene)pivalamide (6m)<smiles>CC(C)=CS(=O)(=NC(=O)C(C)(C)C)C(C)(C)C</smiles>

HPLC analysis: Daicel CHIRALPAK IC, hexane $/ \mathrm{PrOH}=3 / 1$, flow rate $0.75 \mathrm{~mL} / \mathrm{min}$, $\lambda=210 \mathrm{~nm}$, retention time: $11.7 \mathrm{~min}$ (minor) and $14.9 \mathrm{~min}$ (major).

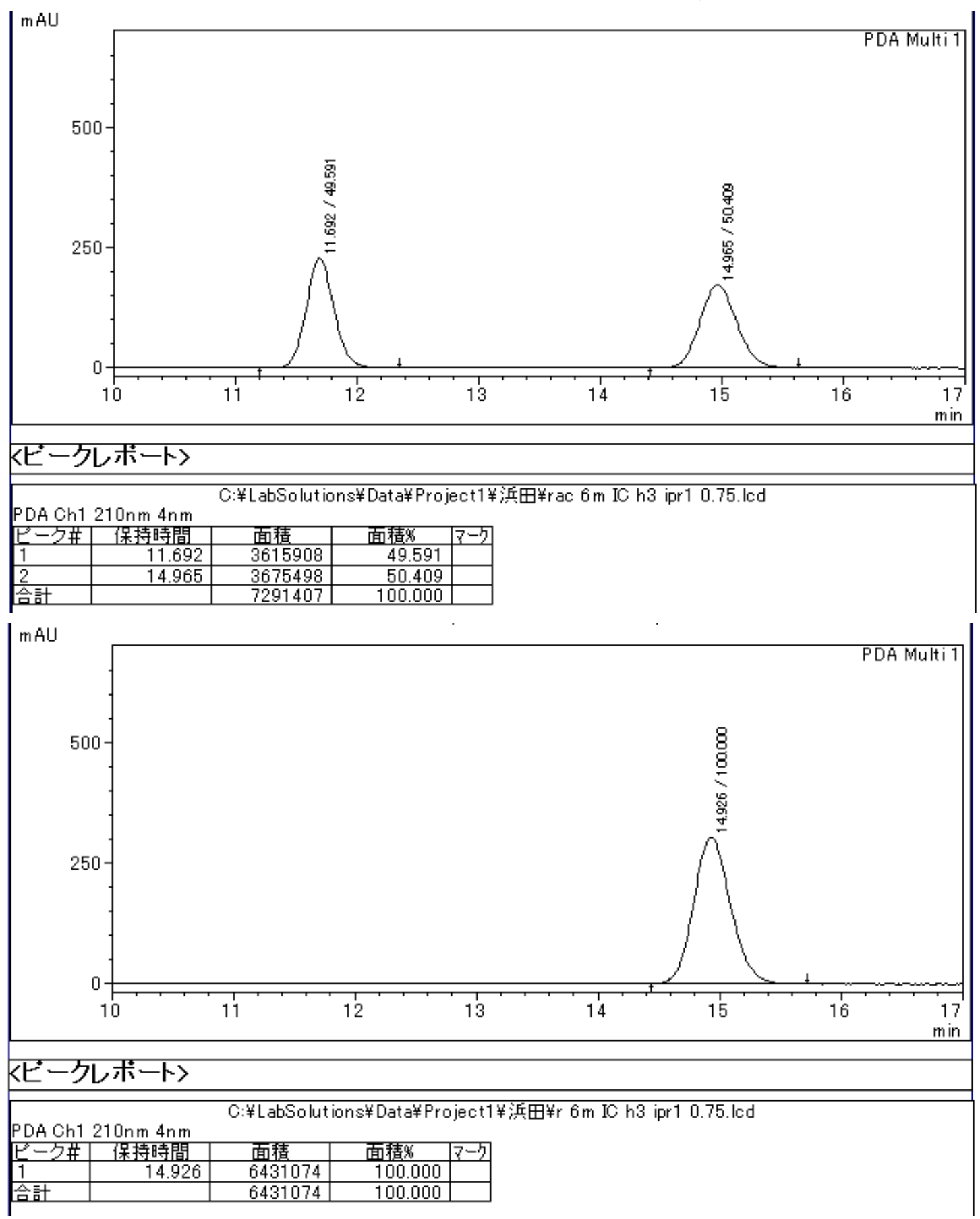


(S)-N-(Butylsulfinyl)pivalamide (7)<smiles>CC(C)(C)C(=O)NS([13CH3])=O</smiles>

HPLC analysis: Daicel CHIRALPAK IC, hexane $/ \mathrm{PrOH}=1 / 1$, flow rate $0.75 \mathrm{~mL} / \mathrm{min}$, $\lambda=206 \mathrm{~nm}$, retention time: $11.3 \mathrm{~min}$ (major) and $13.1 \mathrm{~min}$ (minor).
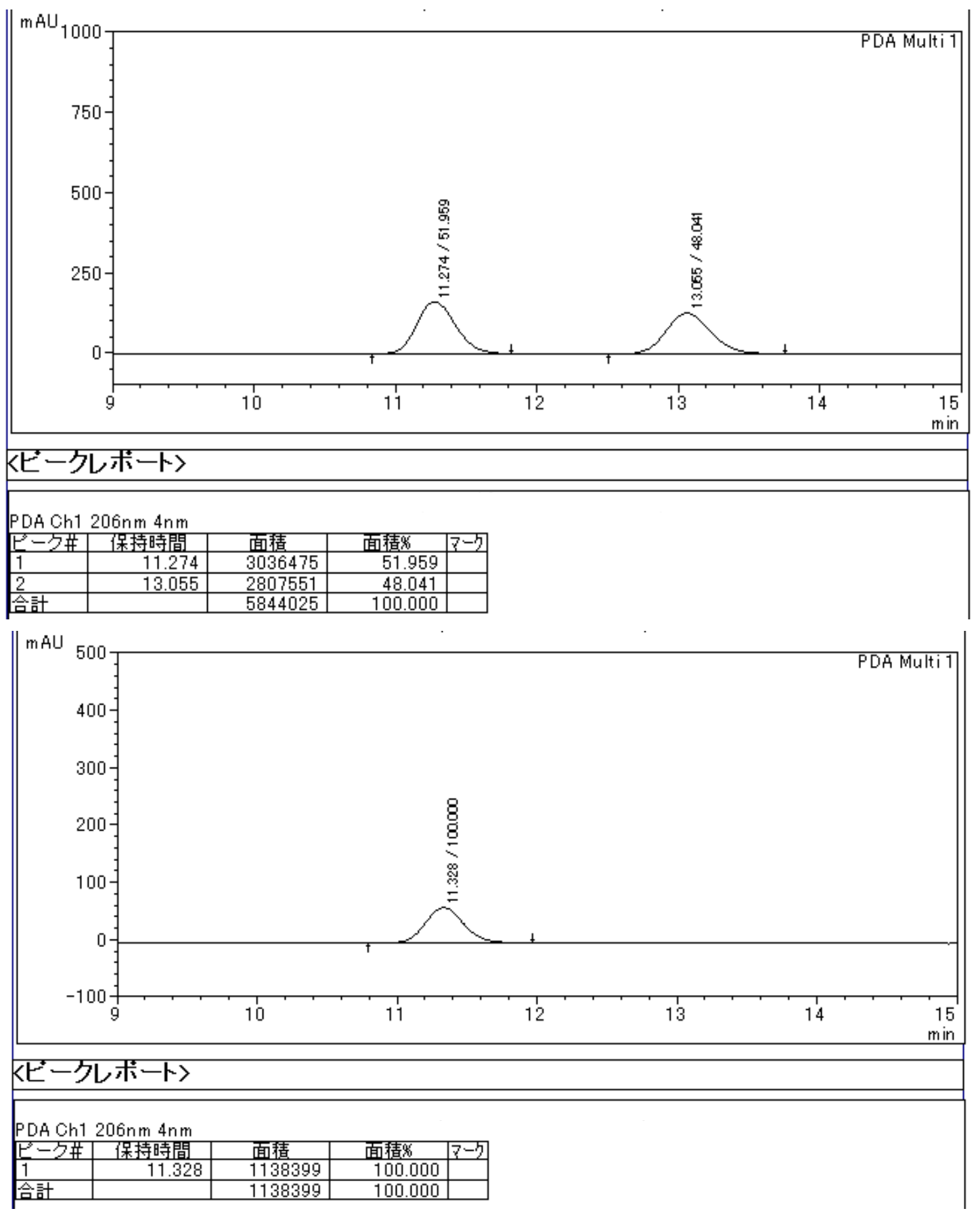
(S,E)-N-((1-Propen-1-yl)sulfinyl)pivalamide (8)<smiles>C/C=C/S(=O)NC(=O)C(C)(C)C</smiles>

HPLC analysis: Daicel CHIRALPAK IC, hexane $/ i \mathrm{PrOH}=1 / 1$, flow rate $0.75 \mathrm{~mL} / \mathrm{min}$, $\lambda=210 \mathrm{~nm}$, retention time: $13.6 \mathrm{~min}$ (major) and $16.3 \mathrm{~min}$ (minor).
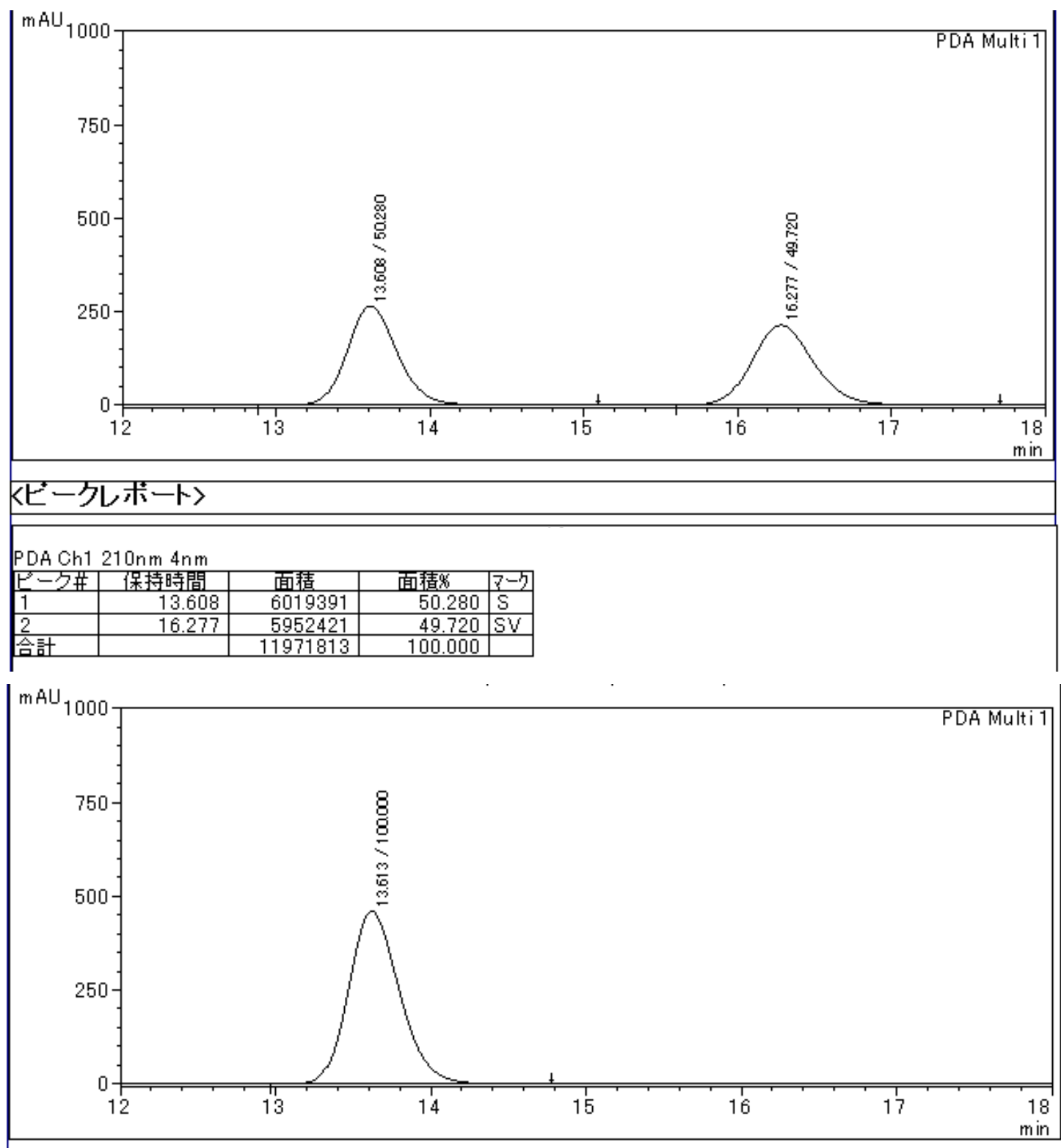

〈ビークしボート〉

\begin{tabular}{|c|c|c|c|c|}
\hline E-万\# & 保持時間 & 面䑶 & 面䞍犷 & $7-5$ \\
\hline 1 & 13.613 & 10549877 & 100.000 & $\mathrm{~S}$ \\
\hline 合計 & & 10549877 & 100.000 & \\
\hline
\end{tabular}




\section{(S)-N-(Butyl(ethyl)(oxo)- $\lambda^{6}$-sulfanylidene)pivalamide (9)}<smiles>CCS(=O)(=O)NC(=O)C(C)(C)C</smiles>

HPLC analysis: Daicel CHIRALPAK IE, hexane $/ \mathrm{PrOH}=1 / 1$, flow rate $0.60 \mathrm{~mL} / \mathrm{min}$, $\lambda=210 \mathrm{~nm}$, retention time: $8.8 \mathrm{~min}$ (major) and $9.3 \mathrm{~min}$ (minor).

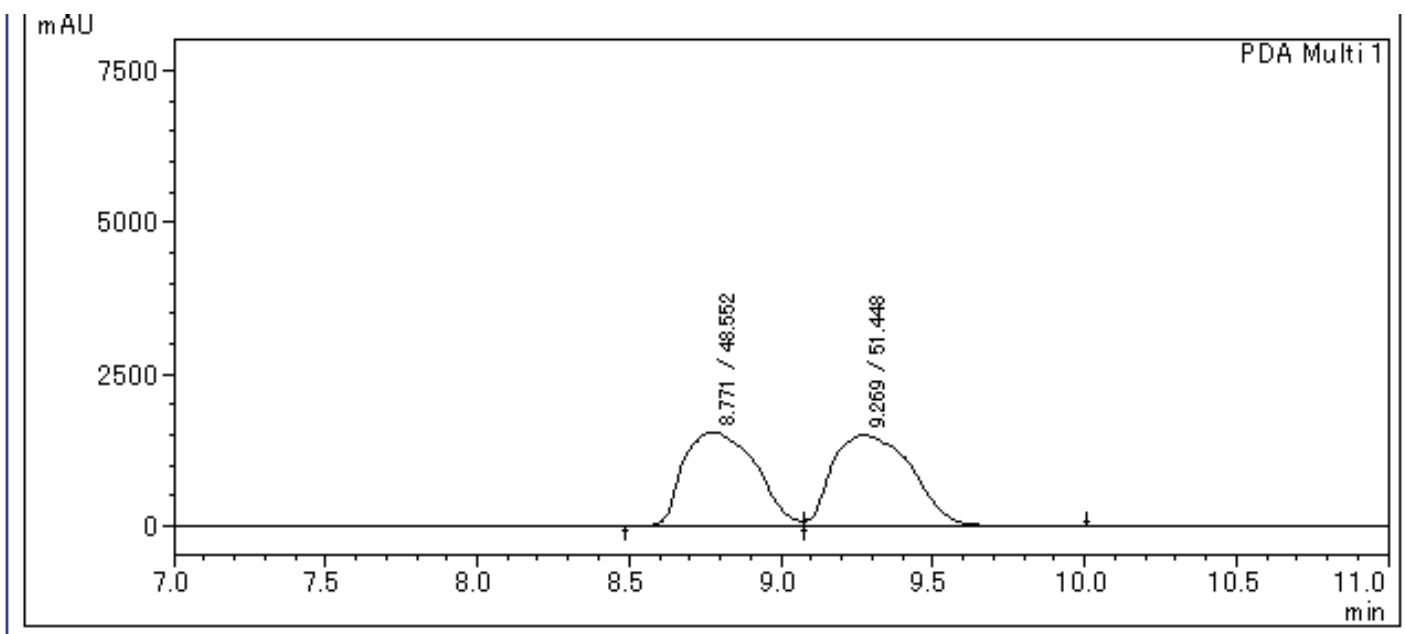

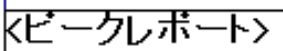

\begin{tabular}{|c|c|c|c|c|}
\hline \multicolumn{5}{|c|}{ PDA Ch1 210nm 4nm } \\
\hline E゙ーク\# & 保持時間 & 面猜 & 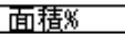 & $7-5$ \\
\hline 1 & 8.771 & 25493061 & 48.552 & \\
\hline 2 & 9.269 & 27013234 & 51.448 & V \\
\hline 合計 & & 52506295 & 100.000 & \\
\hline
\end{tabular}

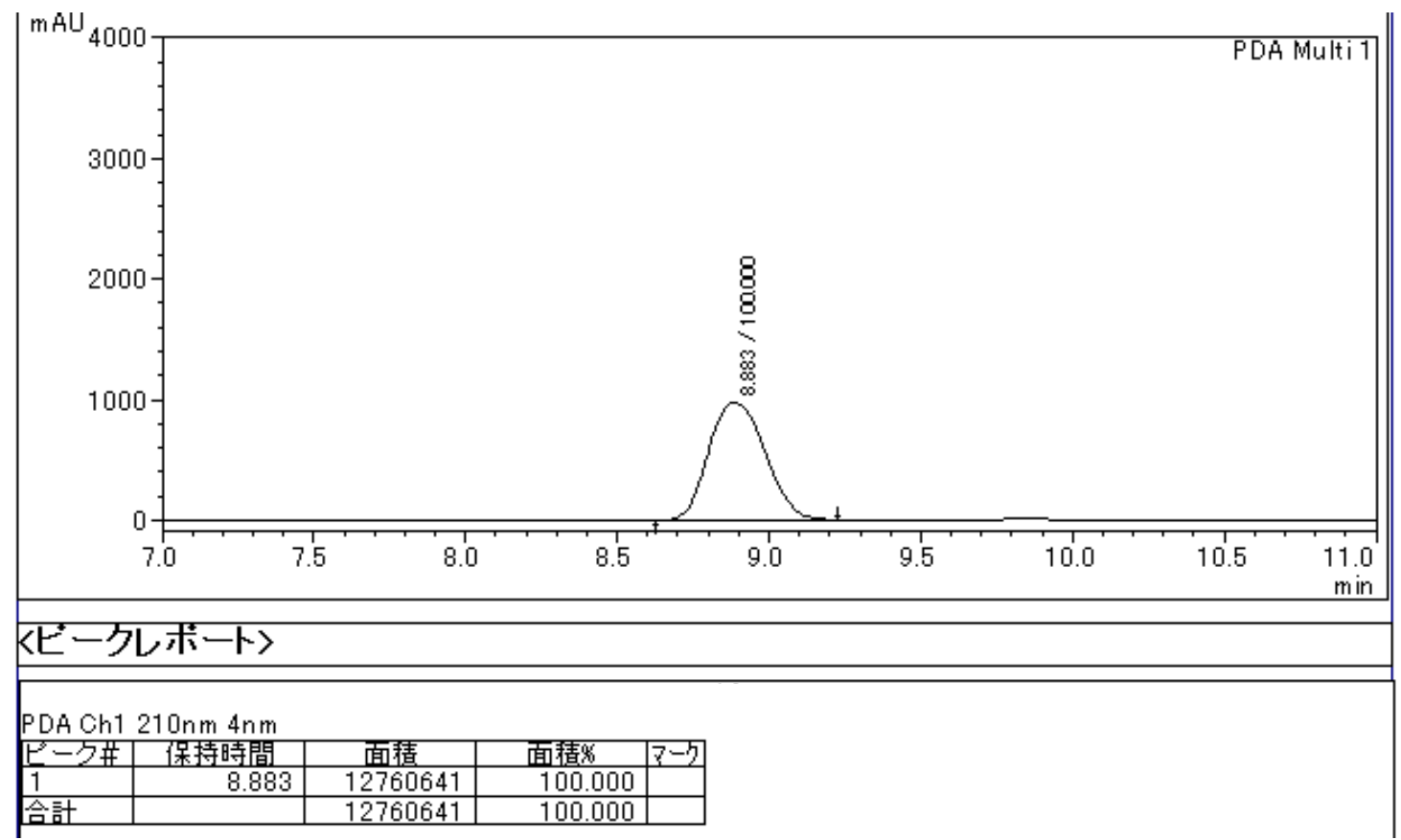


(S)-N-(p-Tolylsulfinyl)pivalamide (10)<smiles>Cc1ccc(S(=O)NC(=O)C(C)(C)C)cc1</smiles>

HPLC analysis: Daicel CHIRALPAK IE, hexane $/ \mathrm{PrOH}=1 / 1$, flow rate $0.75 \mathrm{~mL} / \mathrm{min}$, $\lambda=254 \mathrm{~nm}$, retention time: $9.3 \mathrm{~min}$ (major) and $10.0 \mathrm{~min}$ (minor).

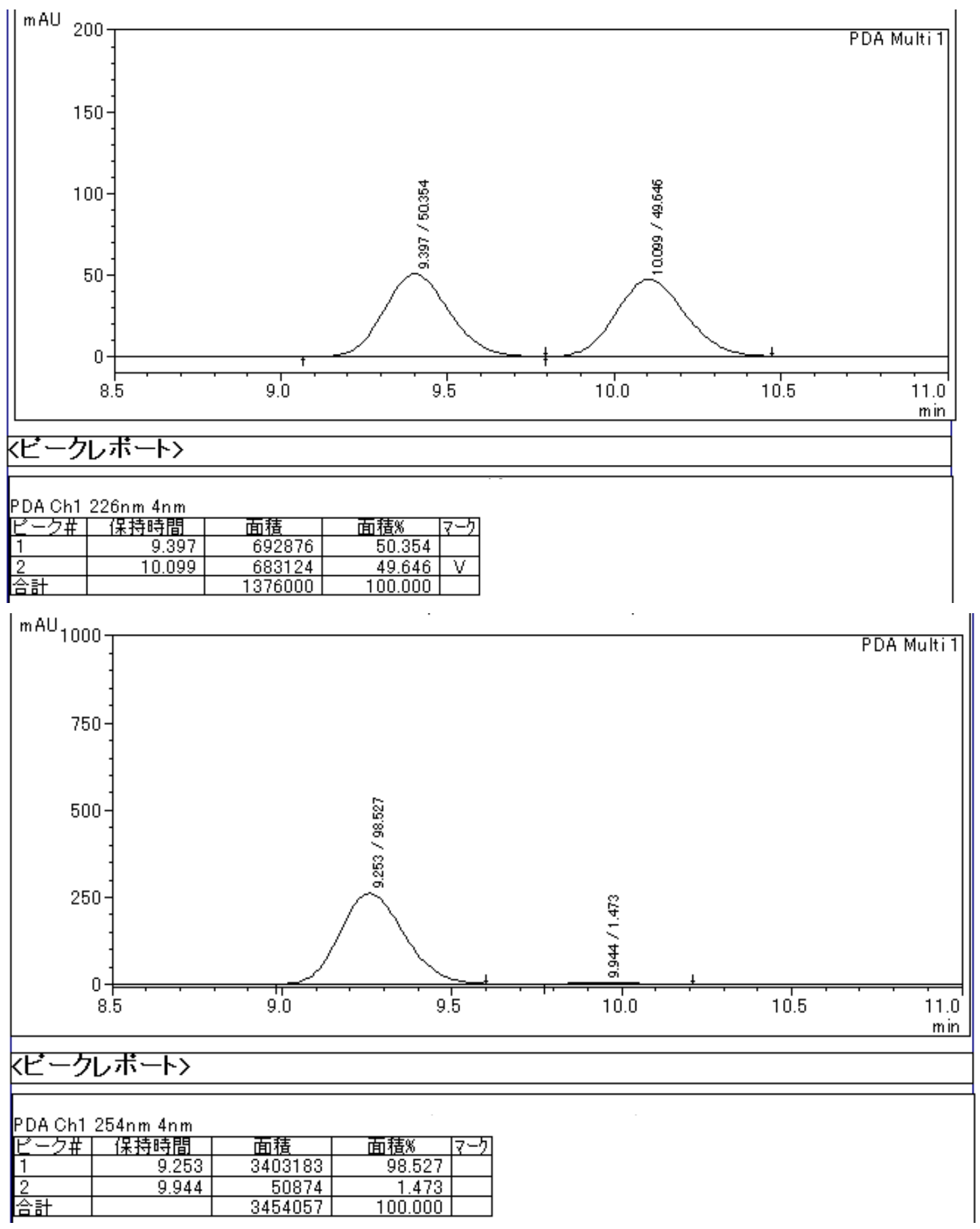


(S)-N-(Ethyl(oxo)(p-tolyl)- $\lambda^{6}$-sulfanylidene)pivalamide (11)<smiles>CCC(=O)NS(=O)(=O)c1ccc(C)cc1</smiles>

HPLC analysis: Daicel CHIRALPAK IG, hexane $/ \mathrm{PrOH}=1 / 1$, flow rate $0.75 \mathrm{~mL} / \mathrm{min}$, $\lambda=226 \mathrm{~nm}$, retention time: $9.6 \mathrm{~min}$ (major) and $13.7 \mathrm{~min}$ (minor).
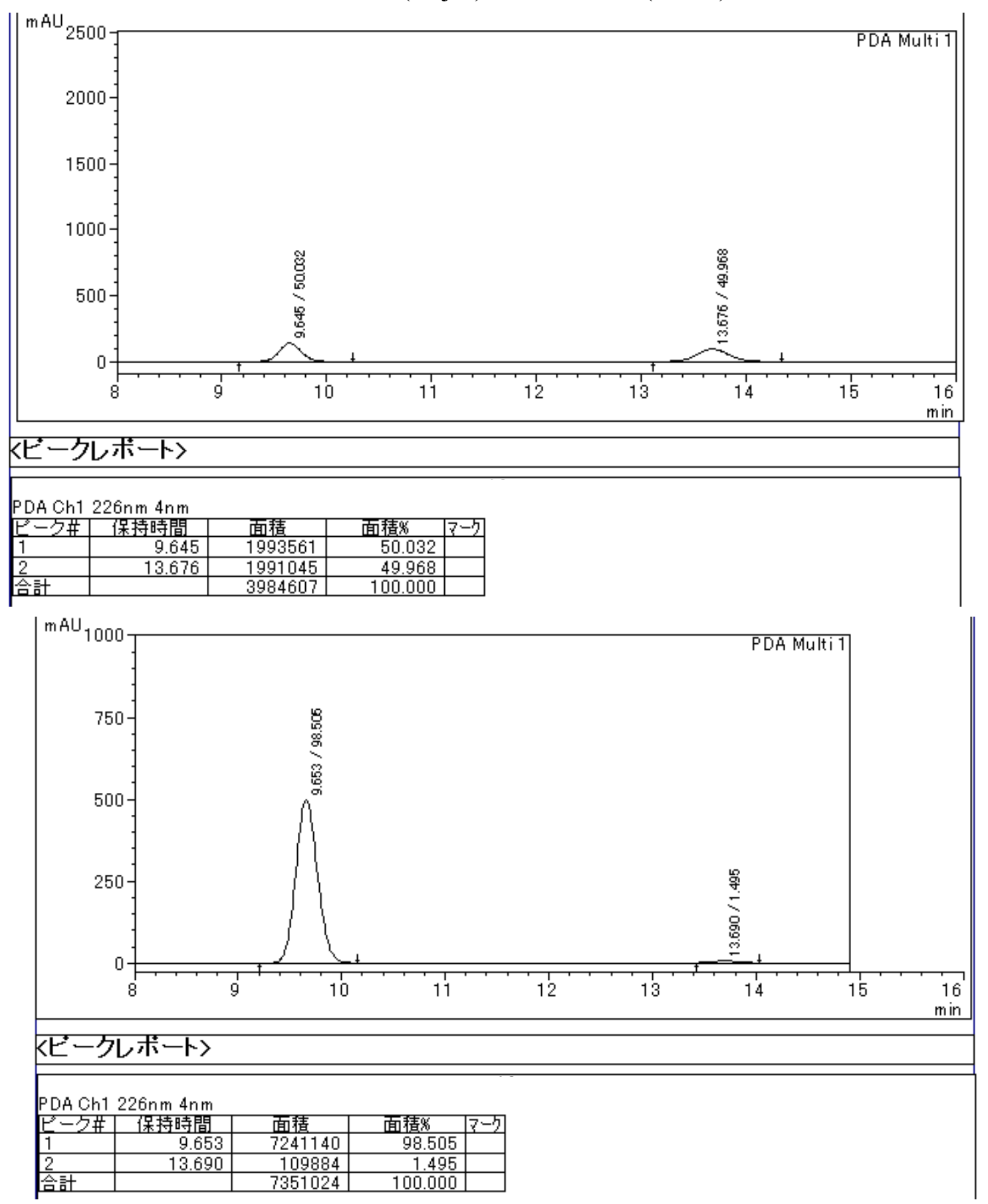


\section{ORTEP drawing of compound 4}

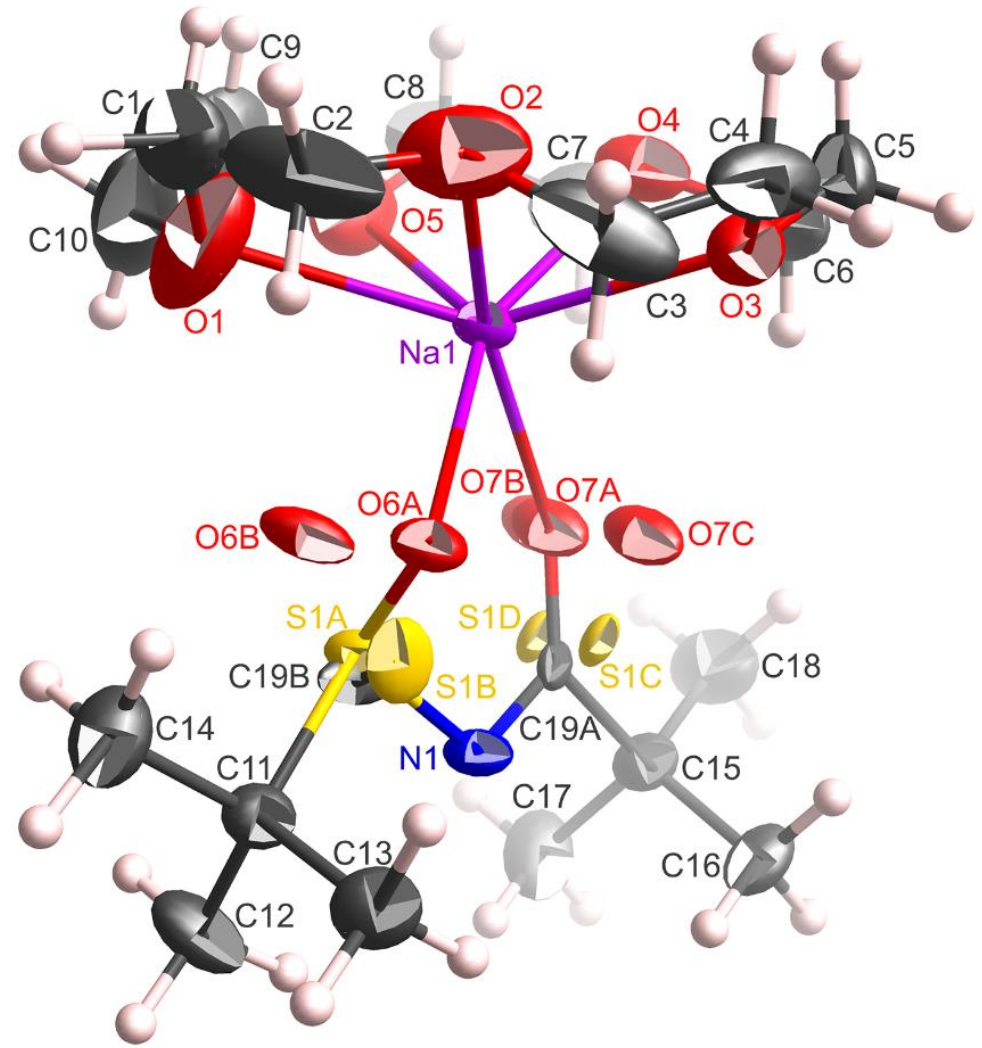

Figure S1. ORTEP drawing of 4 (50\% probability) 